\title{
A Tutorial on Multiple Extended Object Tracking
}

This paper was downloaded from TechRxiv (https://www.techrxiv.org).

\section{LICENSE}

CC BY 4.0

SUBMISSION DATE / POSTED DATE

03-02-2022 / 10-02-2022

CITATION

Granström, Karl; Baum, Marcus (2022): A Tutorial on Multiple Extended Object Tracking. TechRxiv. Preprint. https://doi.org/10.36227/techrxiv.19115858.v1

DOI

10.36227/techrxiv.19115858.v1 


\title{
A Tutorial on Multiple Extended Object Tracking
}

\author{
KARL GRANSTRÖM ${ }^{1}$ and MARCUS BAUM ${ }^{2}$ \\ ${ }^{1}$ Embark Trucks, San Francisco, CA, USA. His contributions to the article were written while he was with the Department of Signals and Systems, Chalmers \\ University of Technology, Gothenburg, Sweden \\ ${ }^{2}$ Institute of Computer Science and Campus Institute Data Science, University of Goettingen, Goettingen, Germany \\ Corresponding author: Marcus Baum (e-mail: marcus. baumecs. uni-goettingen. de).
}

\begin{abstract}
This tutorial introduces state-of-the-art methods for tracking multiple spatially extended objects based on unlabeled noisy point clouds, e.g., from RADAR or LIDAR sensors. In the first part, the focus lies on tracking a single extended object, i.e., the objective is to simultaneously estimate the shape and position of a moving object based on spatially distributed noisy detections. Model-based approaches for tracking elliptical and star-convex shaped objects are treated. Furthermore, recent learning-based approaches are discussed, which learn the spatial distribution of detections from real data. The second part considers the track management and data association problem, i.e., the initialization and termination of tracks as well as the association of detections to objects. After an in-depth analysis of the data association problem for extended objects, various data association approaches are discussed. Finally, recent frameworks for multiple extended object tracking are introduced such as the random finite set-based Poisson multi-Bernoulli mixture framework.
\end{abstract}

INDEX TERMS Multi-object tracking, extended object tracking, state estimation, sensor fusion, radar, lidar, environment perception, autonomous systems

\section{INTRODUCTION}

Environment perception for autonomous systems such as self-driving vehicles [1] involves the problem of detecting and tracking moving objects in the surveillance area. For this purpose, scan-based sensors such as RAdio Detection And Ranging (RADAR) [2], [3] and LIght Detection And Ranging (LIDAR) [4] are becoming increasingly important. These sensors emit a beam and scan the environment in order to create a point cloud based on the received reflections. As individual point detections in the point cloud are noisecorrupted and their reflection origins are unknown, algorithms that filter out sensor noise and extract (labeled) object tracks are required. This problem is in general known as Multiple Object Tracking (MOT), see for example [5], [6].

In traditional MOT applications, e.g., air surveillance, at most a single detection (i.e., measurement) per target object is received by the sensor. However, in many recent applications such as autonomous driving, this is not the case as the sensor resolution is high enough to provide multiple detections per target object, see Fig. 1 for an illustration. In general, the tracking of multiple objects, where a single object can result in multiple (unlabeled) spatially distributed detections is called Multiple Extended Object Tracking (МЕOT). In this case, the spatial extent of the object cannot be neglected and must be determined by the tracking algorithm. Furthermore, data association and track

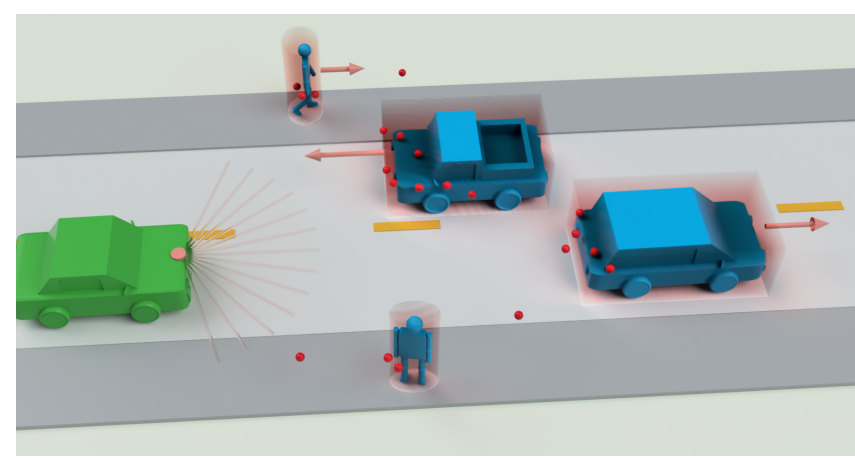

FIGURE 1. Example of multiple extended object tracking: a self-driving vehicle (green) equipped with a sensor is navigating through an urban environment. The sensor of the self-driving vehicle (such as a LIDAR or RADAR device) scans the environment and outputs detections (red dots). Based on these detections, the objective of a multiple extended object tracker is to determine the location, shape, size and motion (e.g., velocity) of each object. In this figure, the vehicles are approximated as boxes and pedestrians as elliptical cylinders. The velocities are indicated by the red arrows.

management (i.e., the initialization and termination of tracks) becomes significantly more complex than for point targets.

Due to the steadily increasing resolution of modern sensors, MEOT recently became a key problem for the environment perception of autonomous systems. Based on advances in nonlinear estimation, random set theory, and machine learning, huge progress has been achieved in MEOT in the 


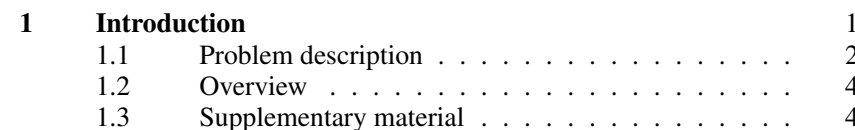

$2 \quad$ Single extended object tracking: Description and modeling 4 2.1 State vector ............... . . . . 4

$2.2 \quad$ Dynamic model . . . . . . . . . . . . . . . . 5

$2.3 \quad$ Measurement model

$2.4 \quad$ Single extended object tracker

$3 \quad$ Elliptic extent approaches

$3.1 \quad$ Random matrix approaches . . . . . . . . . . . . . . 7

3.2 Explicit parameterization . . . . . . . . . . . 8

$3.3 \quad$ Further extensions, models and methods. . . . . . . 10

$4 \quad$ Star-convex approaches 10

4.1 Random hypersurface model . . . . . . . . . . . . 11

$4.2 \quad$ Gaussian process approach $\quad$. . . . . . . . . . . . 12

$4.3 \quad$ Cartesian-defined star-convex models . . . . . . . . . 13

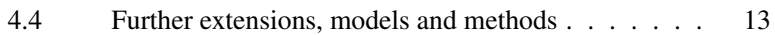

5 Gaussian mixture-based approaches 14

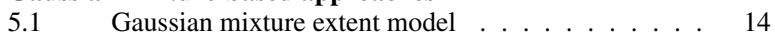

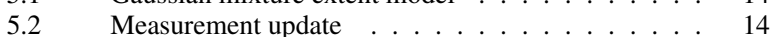

$5.3 \quad$ Further extensions, models and methods. . . . . . . 15

$6 \quad$ Metrics for comparing extent estimates 15

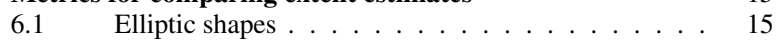

6.2 Star-convex shapes . . . . . . . . . . . . . 15

\begin{tabular}{|lll}
7 & Multiple extended object tracking: Description and modeling 16
\end{tabular} 7.1 Random finite sets . . . . . . . . . . . 16 Standard models for multiple extended object tracking 17 7.3 Problem definition: Multiple extended object tracking 18

$8 \quad$ Analysis of extended object data association 19

8.1 The data association problem . . . . . . . . . . . . . 19

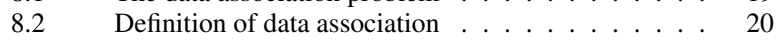

\begin{tabular}{llll}
\hline 8.3 & Complexity of data association & . . . . . . . . . . . 20 & 20
\end{tabular}

$8.4 \quad$ Approximation error upper bound . . . . . . . . . . 21

$9 \quad$ Extended object data association methods 22

9.1 Gating . ................. 22

$9.2 \quad$ Clustering followed by assignment . . . . . . . 22

9.3 Sampling ...................... 23

$9.4 \quad$ Marginal association probabilities . . . . . . . . . 25

$10 \quad$ Algorithms for tracking multiple extended objects 26

10.1 PHD filter . . . . . . . . . . . . . . 26

10.2 $\quad$ CPHD filter . . . . . . . . . . . . . . 27

10.3 Poisson multi-Bernoulli mixture conjugate prior. . . 27

$10.4 \quad$ Labeled multi-Bernoulli mixture conjugate prior . . 29

10.5 Joint probabilistic data association . . . . . . . . 29

10.6 Probabilistic multi hypothesis tracker . . . . . . . . 30

10.7 Graph-based approaches . . . . . . . . . . . . 30

11 Conclusions

FIGURE 2. Structure of this tutorial.

last years. The objective of this tutorial article is to provide an intuitive introduction into the state-of-the-art in MEOT, where also an overview of recent research results is given.

This tutorial complements and updates our previous overview article [7]. The focus of [7] lies on Single Extended Object Tracking (SEOT), i.e., on the extended object problem formulation, the random matrix approach for elliptical extended objects, and applications. This tutorial adds to [7], among others, the newest methods for elliptical extended objects, a more comprehensive description of star-convex approaches, learning-based approaches, and a significantly more elaborate discussion of data association and multiple extended object tracking methods, including the newest approaches based on Random Finite Set (RFS) theory.

The tutorial aims at readers who are familiar with basic (nonlinear) estimation techniques (see, e.g., [8]) and standard multi-object tracking methods (see, e.g., [5] or [6]).

\subsection{PROBLEM DESCRIPTION}

In the following, we introduce the MEOT problem and give an overview of the involved challenges. Consider the example in Figure 11, where a self-driving vehicle is shown in an urban environment with other objects, in this case cars and pedestrians. For safe and robust operation, it is important that the self-driving vehicle is able to detect and track the objects that are in its vicinity, i.e., it must solve the multiple (extended) object tracking problem. For this purpose, it is equipped with passive sensors such as cameras but also scanbased sensors such as RADAR or LIDAR devices. In particular, scan-based sensors directly provide detections of objects in the environment. Each detection represents a reflection of the omitted beam, i.e., an obstacle. In case the spatial extent of an object is larger than the sensor resolution, multiple detections from different sources on an object can be received.

Typically in MEOT, one has to deal with the following challenges:

- The objects are not stationary but can move around.

- The number of objects is unknown and time varying.

- It is unknown which objects are detected, and which are not, and there are also false measurements.

- The data association is unknown, i.e., it is unknown what the source of a detection is (an object or clutter).

- For the detected objects, it is not known how many detections they caused, and it is not known how the measurements are distributed around the object.

- The shapes and dimensions of the objects are unknown.

MEOT is typically applied to data from sensors that have a limited Field Of View (FOV). For example, an automotive radar has some opening azimuth angle and some maximum range, which together define a FOV in the form of a circle segment. With multiple sensors, the union of the fields of view defines the so called surveillance area. Regardless if the sensor is stationary or mobile, the objects are not guaranteed to remain in the surveillance area. At any time step, there is a possibility that some new objects will enter, or appear in, the surveillance area, and that some of the existing objects will leave, or disappear from, the surveillance area. In other words, the number of objects is not just unknown, but also time varying. To handle the time-varying number of objects, MEOT algorithms include models of so called object birth and object death.

The unknown object motion is described with motion models. An extended object motion model describes both 


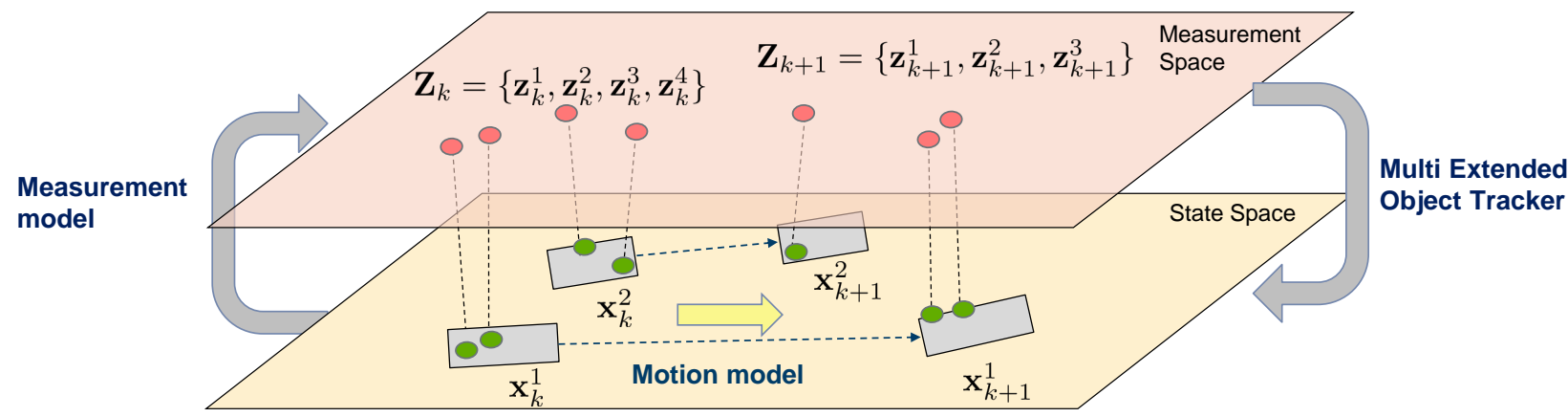

FIGURE 3. Illustration of the Multi-Extended Object Tracking (МЕOT) problem with two target objects: The states $\mathbf{x}_{k}^{1}$ and $\mathbf{x}_{k}^{2}$ of the two target objects at time $k$ are gathered in a set $\mathbf{X}_{k}=\left\{\mathbf{x}_{k}^{1}, \mathbf{x}_{k}^{2}\right\}$. At each time $k$, each object may result in multiple detections, i.e., measurements. For example, in this illustration, the measurement set at time $k$ is $\mathbf{Z}_{k}=\left\{\mathbf{z}_{k}^{1}, \mathbf{z}_{k}^{2}, \mathbf{z}_{k}^{3}, \mathbf{z}_{k}^{4}\right\}$ and consists of four measurements $\mathbf{z}_{k}^{1}, \mathbf{z}_{k}^{2}, \mathbf{z}_{k}^{3}$ and $\mathbf{z}_{k}^{4}$.

how the object's position and kinematics evolve over time, and how the extent evolves over time. A common approach is to use a standard motion model, e.g., constant velocity or coordinated-turn, and to assume that the extent is aligned rigidly to the direction of motion. The latter assumption holds for most objects in most scenarios; one exception is objects that are experiencing significant drifting, however, such extreme motion is rare in extended object applications.

The input data to an MEOT algorithm are object detections, which are obtained by processing the raw sensor data with a detector. An inherent property of a detector is that it is susceptible to two types of error: misdetections (false negatives) and false detections (false positives). A misdetection occurs when an object is located in the surveillance area, but is not detected in the sensor data. A false detection occurs when the detector outputs a detection, but in reality there is no object. Furthermore, in MEOT, the data association is not known, i.e., given a set of detections, it is not known which detections are from which object, and which detections are clutter. This poses a considerable challenge in MEOT as multiple detections can come from the same object. Finally, a major challenge is that it is not known which point exactly was detected on an object. The underlying source of a detection depends on many aspects such as the sensorto-object geometry and the properties of the target object. Hence, already the Single Extended Object Tracking (SEOT) is challenging. In SEOT, the task is to simultaneously estimate the shape, size, and position of an extended object based on noisy detections of the object.

A more formal illustration of the MEOT problem including the involved quantities is depicted in Fig. 3 The state vector of an object, which (for example) consists of the position, velocity and shape parameters, is denoted as $\mathbf{x}_{k}^{i}$, where $k$ denotes a discrete time index and $i$ is the object index. All objects at time $k$ are gathered in a set

$$
\mathbf{X}_{k}=\left\{\mathbf{x}_{k}^{i}\right\}_{i \in \mathbb{I}}
$$

where objects are indexed by $\mathbb{I}$. The received detections at time $k$ are gathered in the measurement set $\mathbf{Z}_{k}$ and indexed by $m \in \mathbb{M}$, i.e.,

$$
\mathbf{Z}_{k}=\left\{\mathbf{z}_{k}^{m}\right\}_{m \in \mathbb{M}} .
$$

The measurement model relates the set of target objects $\mathbf{X}_{k}$ to the set of measurements $\mathbf{Z}_{k}$ and the motion model specifies the temporal evolution of the set of target objects $\mathbf{X}_{k}$. The task of MEOT is to estimate the object states $\mathbf{X}_{1}, \ldots, \mathbf{X}_{k}$ based on the measurement sets $\mathbf{Z}_{1}, \ldots, \mathbf{Z}_{k}$. For this purpose, it is common to employ recursive Bayesian filters that sequentially incorporate the incoming measurements and update a probability density function for the object states based on Bayes' rule. In the context of MOT, the RFS framework [9], [10], which allows to systematically deal with unlabeled and unordered quantities, becomes increasingly important.

According to the above discussion, the MEOT problem can be decomposed into three sub-problems:

- Unknown number of objects: The number of objects $n_{k}=\left|\mathbf{X}_{k}\right|$ in the environment varies over time, i.e., new objects must be initialized and existing tracks must be terminated. Traditionally, track initialization and termination is also called track management.

- Unknown data association: Given a specific object $\mathbf{x}_{k}^{i} \in$ $\mathbf{X}_{k}$, it is unknown which subset $\mathbf{W}_{k}^{i} \subseteq \mathbf{Z}_{k}$ of the measurements originates from the target $\mathbf{x}_{k}^{i}$. The process of assigning measurements to objects is traditionally called data association. A special challenge in MEOT is that the number of possible associations is extremely large as multiple measurements can come from the same object.

- Unknown shape: The measurements are noise-corrupted and the exact point on the object that was detected is unknown. This means, even if the measurement-toobject assignment is given, i.e., it is known that the measurements $\mathbf{W}_{k} \subseteq \mathbf{Z}_{k}$ come from the object $\mathbf{x}_{k}$, it is challenging to estimate the state $\mathbf{x}_{k}$.

Note that the MEOT problem is significantly different from the typical vision-based object tracking setting [11]-[13]. For example, in the tracking-by-detection approach, the objects are detected, e.g., by a neural network, and subsequently the detections are associated between frames. In MEOT, however, multiple (point) detections of an object are directly received from the sensor in a single scan. 
TABLE 1. Notation

- Small non-bold letters represent scalar values, e.g., $\alpha, \beta$ and $\gamma$

- Small bold letters represent vectors, e.g., $\mathbf{x}, \mathbf{z}$ and $\boldsymbol{\xi}$

- The dimension of the vector $\mathbf{x}$ is denoted as $\operatorname{dim}(\mathbf{x})$

- Capital non-bold letters represent matrices, e.g., $X, H$ and $F$

- $\operatorname{Tr}(F)$ denotes the trace of $F$

- Capital bold letters denote sets, e.g., $\mathbf{X}, \mathbf{Z}$ and $\mathbf{C}$

- The determinant of the matrix $V$ is denoted as $|V|$

- The number of elements in the set $\mathbf{X}$ (also denoted as cardinality) is written as $|\mathbf{X}|$

- The identity matrix with dimensions $m \times m$ is $I_{m}$

- The Kronecker delta function is defined as $\Delta_{a}(x)= \begin{cases}1 & x=a \\ 0 & x \neq a\end{cases}$

- The inner product of $a(x)$ and $b(x)$ is $\langle a ; b\rangle=\int a(x) b(x) \mathrm{d} x$

- By definition $h^{\mathbf{X}}:=\prod_{\mathbf{x} \in \mathbf{x}} h(\mathbf{x})$ with $h^{\emptyset}:=1$

- The (possibly empty, mutually disjoint) subsets $\mathbf{X}^{i}, i \in \mathbb{I}$, whose union is $\mathbf{X}$, are written as $\uplus_{i \in \mathbb{I}} \mathbf{X}^{i}=\mathbf{X}$, see [9. Sec. 11.5.3].

- The Binomial coefficient is defined as $\left(\begin{array}{l}a \\ b\end{array}\right)=\frac{a !}{b !(a-b) !}$

- The Stirling number of the second kind is $\left\{\begin{array}{l}a \\ b\end{array}\right\}=$ $\frac{1}{b !} \sum_{k=0}^{b}(-1)^{b-k}\left(\begin{array}{l}b \\ k\end{array}\right) k^{a}$

- The Bell number of $n$-th order is denoted as $B(n)$

Public data sets suitable for MEOT can be found, for example, in the RADAR and LIDAR data sets of the NuScenes benchmark [14] for autonomous driving, and in the marine radar data set [15] for maritime surveillance.

\subsection{OVERVIEW}

The structure of this tutorial is depicted in Fig. 2 and the used notation is listed in Table 1

Section 2 to Section 6 are devoted to the single extended object tracking problem, i.e., the question is how to determine the spatial extent of a moving object - given spatially distributed detections of the object. After a description of the single object tracking problem in Section 2, basic methods for estimating elliptic and star-convex shape approximations are discussed in Section 3 and Section 4, respectively. An overview of learning-based approaches that learn the measurement distributions is given in Section 5 Finally, metrics for performance evaluation of EOT methods are elaborated in Section 6

Section 7 to Section 10 treat the challenges that come with multiple extended objects, i.e., in particular data association and track management. The basic concepts required for MEOT are introduced in Section 77. In Section 8, we analyze the complexity of the data association problem, and in Section 9 we review methods for dealing with the data association. An introduction to state-of-the-art MEOT frameworks is then provided in Section 10 . The tutorial ends with a conclusion in Section 11 .

\subsection{SUPPLEMENTARY MATERIAL}

Supplementary material including source code is provided on the following tutorial web-page:

\section{http://meot-tutorial.uni-goettingen.de}

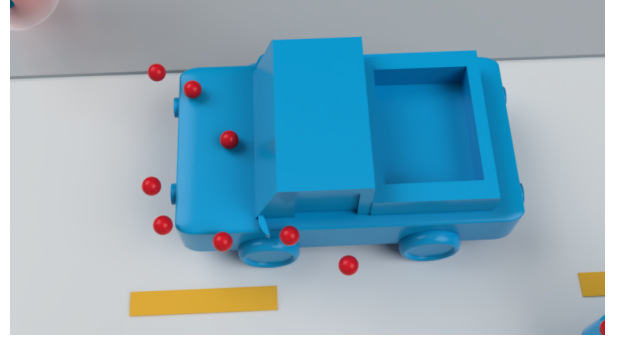

FIGURE 4. Illustration of the (single) extended object tracking problem: A sensor (in this case mounted on the own vehicle) receives multiple spatially distributed detections (red dots) from a moving target object (here a vehicle). The kinematics (e.g., velocity) and spatial extent (including shape and orientation) of the target are unknown and must be estimated.

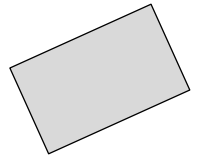

Basic Shape

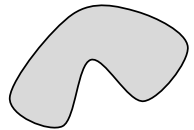

Star-Convex Shape

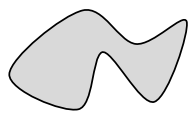

Arbitrary Shape
FIGURE 5. Different shape complexities that are typically distinguished in extended object tracking.

\section{SINGLE EXTENDED OBJECT TRACKING: DESCRIPTION AND MODELING}

The first part of this tutorial focuses on Single Extended Object Tracking (SEOT), i.e., we assume that the available detections all belong to a specific target object, see Fig. 4 The basic task in SEOT is to (recursively) estimate

- the position and kinematics (velocity, heading etc.) of the object, plus

- the spatial extent (i.e., shape, size, and orientation)

based on the available detections. Extended object tracking is in particular challenging in case of a rather low sensor resolution when

- the sensor noise is high compared to the spatial extent of the object, and

- only a few detections are available per scan.

In the remainder of this section, it is shown how a suitable state vector for extended objects can be defined, and how both the temporal evolution and measurement generation can be modeled.

\subsection{STATE VECTOR}

As the spatial extent of the object is unknown and must be estimated, the state vector

$$
\mathbf{x}_{k}=\left[\mathbf{p}_{k}^{\mathrm{T}}, \mathbf{m}_{k}^{\mathrm{T}}\right]^{T}
$$

of an extended object at (discrete) time $k$ consists of

- the extent parameter vector $\mathbf{p}_{k}$ that describes the shape, orientation, and size of the target, and

- the kinematic state $\mathbf{m}_{k}=\left[\mathbf{d}_{k}^{\mathrm{T}}, \ldots\right]^{T}$ with position $\mathbf{d}_{k}$ and (possibly) further parameters such as speed, acceleration, heading, turn-rate (yaw-rate), roll, or pitch.

A suitable choice of the extent parameters depends highly on the considered scenario. Fig. 5 shows shapes of different 
complexity that are usually distinguished in extended object tracking. For example, in case of high sensor resolution, the sensor might be able to provide enough measurements from the object such that a detailed contour can be extracted. However, in case of low sensor resolution, it might only be possible to extract coarse shape information (e.g., orientation, length, and width). Once a specific type of shape has been identified, a suitable parameterization needs to be defined. Depending on the application and sensor resolution, it might also be reasonable to use a precise shape representation but only estimate the orientation, width and length, see for example Section 4.3 .

As an example, consider an elliptical extended object whose state vector could be defined as

$$
\mathbf{x}_{k}=[\underbrace{\alpha_{k}, l_{k, 1}, l_{k, 2}}_{\mathbf{p}_{k}^{\mathrm{T}}}, \underbrace{\mathbf{d}_{k}^{\mathrm{T}}, \mathbf{v}_{k}^{\mathrm{T}}}_{\mathbf{m}_{k}^{\mathrm{T}}}]^{T},
$$

where

- $\alpha_{k}$ is the orientation,

- $l_{k, 1} \in \mathbb{R}$ and $l_{k, 2} \in \mathbb{R}$ are the semi-axes lengths,

- $\mathbf{d}_{k} \in \mathbb{R}^{2}$ is the location and

- $\mathrm{v}_{k} \in \mathbb{R}$ denotes the velocity in direction $\alpha_{k}$.

For a compact formulation of the algorithms that are introduced in this tutorial, we define the matrix $H$ that extracts the position $\mathbf{d}_{k}$ from the kinematic state $\mathbf{m}_{k}$, e.g., for extended targets in two-dimensional space, we get

$$
H=\left[\begin{array}{ll}
I_{2 \times 2} & 0_{2 \times \operatorname{dim}(\mathbf{m})-2}
\end{array}\right]
$$

such that

$$
\mathbf{d}_{k}=H \mathbf{m}_{k} .
$$

\subsection{DYNAMIC MODEL}

The temporal evolution of an extended object can be modeled with a transition density

$$
f_{k+1, k}\left(\mathbf{x}_{k+1} \mid \mathbf{x}_{k}\right),
$$

which specifies the density function of the state at time $k+1$ given the state at time $k$. Standard motion models [16] can often be used for the position, e.g., a constant velocity model for pedestrians, or coordinated turn for cars and bicycles. The extent typically has a (nearly) constant size but might rotate during turns.

\subsection{MEASUREMENT MODEL}

The measurements at time $k$ that belong to the target object with state $\mathbf{x}_{k}$ are gathered in the set

$$
\mathbf{W}_{k}=\left\{\mathbf{z}_{k}^{j}\right\}_{j=1}^{n} .
$$

The measurement model relates the state vector $\mathbf{x}_{k}$ to $\mathbf{W}_{k}$ by means of the single target likelihood

$$
f\left(\mathbf{W}_{k} \mid \mathbf{x}_{k}\right),
$$

which specifies how the measurements are distributed around the object and how many measurements are received from
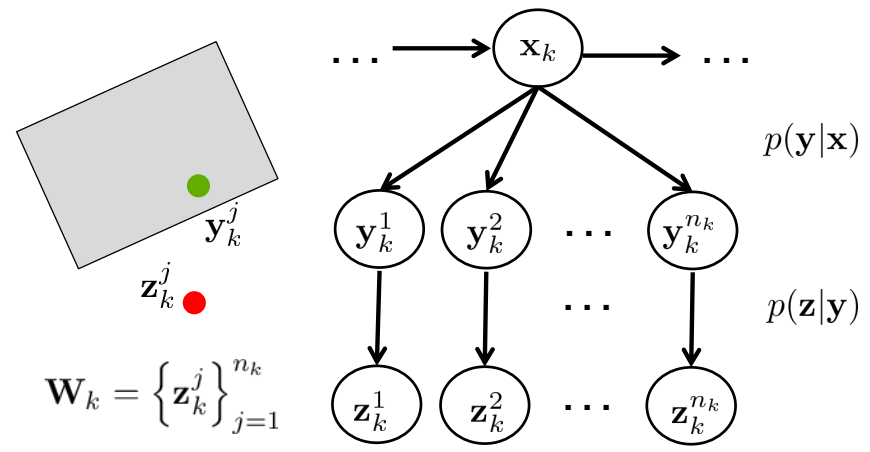

FIGURE 6. Illustration of the spatial distribution model: Each received measurement $\mathbf{z}_{k}^{j}$ at time $k$ stems from a measurement source $\mathbf{y}_{k}^{j}$ on the object, where $p(\mathbf{y} \mid \mathbf{x})$ is the spatial measurement source density, and $p(\mathbf{z} \mid \mathbf{x})$ models sensor noise.

the object. For this purpose, the so-called spatial distribution model [17] is widely-used as it is general enough to precisely model a variety of relevant sensors such as (automotive) RADAR and LIDAR, and at the same time, it allows for deriving efficient (approximate) estimators.

The spatial distribution model assumes that - conditioned on the object the object's position and extent in $\mathbf{x}-$ each individual measurement $\mathbf{z}$ is an independent random draw from the spatial measurement distribution

$$
\phi(\mathbf{z} \mid \mathbf{x}) \quad .
$$

The spatial distribution (8) results from two different aspects. First, due to the spatial extent, the point $\mathbf{y}$ on the object (called measurement source) that is measured by $\mathbf{z}$ is unknown and varies from measurement to measurement. Second, there is additional sensor noise (in the same manner as for point targets). As a consequence, a spatial distribution is usually decomposed as

$$
\phi(\mathbf{z} \mid \mathbf{x})=\int p(\mathbf{z} \mid \mathbf{y}) \cdot p(\mathbf{y} \mid \mathbf{x}) \mathrm{d} \mathbf{y},
$$

where the measurement $\mathbf{z}$ stems from a measurement source $\mathbf{y}$, which is modeled with the spatial measurement source distribution

$$
p(\mathbf{y} \mid \mathbf{x}),
$$

and an additional sensor model is specified by the conditional density function

$$
p(\mathbf{z} \mid \mathbf{y}) .
$$

It is common to assume that the measurement sources are directly measured according to

$$
\mathbf{z}=\mathbf{y}+\mathbf{v},
$$

where $\mathbf{v}$ is additive zero-mean Gaussian noise with covariance matrix $R$. In this case, (11) becomes

$$
p(\mathbf{z} \mid \mathbf{y})=\mathcal{N}(\mathbf{z} ; \mathbf{y}, R) .
$$

The spatial distribution specifies the spatial spread of each individual measurement across the target. The number of 


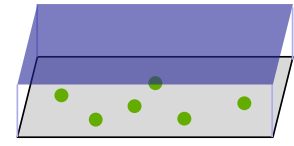

Across

surface

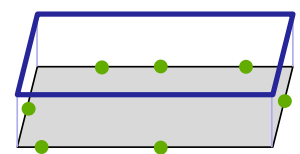

Along

contour

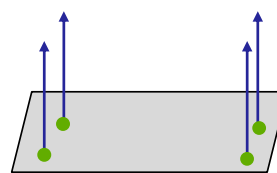

Set of reflec-

tion points

FIGURE 7. Spatial densities can model many relevant reflection patterns, e.g., measurements that come from the entire surface, measurements that come only from the contour, and measurements from a finite set of reflections centers. The spatial density is plotted in blue. The green dots represent some example draws from the spatial distribution.

measurements that is received in a scan is often assumed to be Poisson distributed with state dependent Poisson rate $\kappa\left(\mathbf{x}_{k}\right)$. In total the measurement set is an Inhomogeneous Poisson Point Process (PPP) according to

$$
p\left(\mathbf{W}_{k} \mid \mathbf{x}_{k}\right)=e^{-\lambda\left(\mathbf{x}_{k}\right)} \lambda\left(\mathbf{x}_{k}\right)^{\left|\mathbf{W}_{k}\right|} \prod_{\mathbf{z}_{k}^{j} \in \mathbf{W}_{k}} \phi\left(\mathbf{z}_{k}^{j} \mid \mathbf{x}_{k}\right) .
$$

A visualization of the spatial distribution model is shown in Fig. 6. As illustrated in Fig. 7, a spatial distribution model can model measurements that

- stem from the object contour (such as from a LIDAR sensor),

- cover the entire surface (e.g., from a RADAR), or

- originate from a finite set of reflection centers.

For scan-based sensors such as LIDAR or RADAR, the assumption that the measurements sources are random, conditioned on the state, seems (at the first sight) unreasonable as the measurement source is determined by the intersection of the beam with the target object. However, as the exact surface properties of the target object etc. are unknown, it turns out that it is usually impossible to exactly predict the measurement source. Furthermore, as the spatial distribution explicitly models individual measurements, it can be used for scenarios with a low number of measurements from an object. For example, it even incorporates the case of only one measurement per scan. For this reason, the spatial distribution model is suitable in many real-world settings.

We also note that shape estimation is most challenging for the case of measurements that come from the complete target surface. Consider a circle as an example: If the measurements only come from the contour, three (noise-free) measurements are sufficient to determine the circle parameters uniquely. However, if the measurements may come from the entire circular disc, three measurements intuitively only specify a "lower" bound for the radius.

\subsection{SINGLE EXTENDED OBJECT TRACKER}

The task of a (recursive Bayesian) single extended object tracker is to recursively determine the posterior density of the state vector (3) for time $k$ given the available measurements up to time $k$, i.e.,

$$
p\left(\mathbf{x}_{k} \mid \mathbf{W}_{1: k}\right),
$$

where $\mathbf{W}_{1: k}$ denotes all measurement sets from time 1 to time $k$, i.e.,

$$
\mathbf{W}_{1: k}:=\left(\mathbf{W}_{1}, \ldots, \mathbf{W}_{k}\right) \text {. }
$$

A recursive Bayesian filter consists of alternating time and measurement update steps. The time update step calculates the prediction

$$
\begin{aligned}
& p\left(\mathbf{x}_{k} \mid \mathbf{W}_{1: k-1}\right)= \\
& \quad \int f_{k, k-1}\left(\mathbf{x}_{k} \mid \mathbf{x}_{k-1}\right) \cdot p\left(\mathbf{x}_{k-1} \mid \mathbf{W}_{1: k-1}\right) \mathrm{d} \mathbf{x}_{k-1}
\end{aligned}
$$

based on the filter density $p\left(\mathbf{x}_{k-1} \mid \mathbf{W}_{1: k-1}\right)$ for time $k-1$.

The measurement update step employs the sensor data $\mathbf{W}_{k}$ from time $k$ to calculate the posterior according to Bayes rule

$$
p\left(\mathbf{x}_{k} \mid \mathbf{W}_{1: k}\right)=\frac{p\left(\mathbf{W}_{k} \mid \mathbf{x}_{k}\right) \cdot p\left(\mathbf{x}_{k} \mid \mathbf{W}_{1: k-1}\right)}{p\left(\mathbf{W}_{k} \mid \mathbf{W}_{1: k-1}\right)}
$$

based on the likelihood function (14) and the prediction (17).

As the spatial distribution in Eq. (9) involves an integral that, in general, cannot be solved in closed-form, single extended object tracking is an especially challenging (potentially high-dimensional) nonlinear estimation problem. Closed-form solutions are only available for special cases (e.g., elliptic targets, see Section 3) and basic nonlinear estimation algorithms such as the Unscented Kalman Filter (UKF) [18] or particle filters [8] cannot be applied directly.

\section{ELLIPTIC EXTENT APPROACHES}

Elliptic shape models are widely-used for extended object tracking as the ellipse parameters provide relevant information about the target extent that can even be determined in challenging scenarios involving sharply maneuvering objects, high sensor noise, or a low number of detections. Furthermore, in contrast to rectangles, ellipses are mathematically more convenient to describe, e.g., they can be written as an implicit equation, and they can be interpreted as multivariate Gaussians.

In general, the contour of an ellipse with center $\mathbf{d} \in \mathbb{R}^{2}$ can be represented by the set of all points $\mathbf{y}$ that satisfy the implicit equation

$$
(\mathbf{y}-\mathbf{d})^{\mathrm{T}} X^{-1}(\mathbf{y}-\mathbf{d})=1,
$$

where

$$
X=\underbrace{\left[\begin{array}{cc}
\cos \alpha & -\sin \alpha \\
\sin \alpha & \cos \alpha
\end{array}\right]}_{:=\operatorname{Rot}(\alpha)}\left[\begin{array}{cc}
l_{1}^{2} & 0 \\
0 & l_{2}^{2}
\end{array}\right]\left[\begin{array}{cc}
\cos \alpha & -\sin \alpha \\
\sin \alpha & \cos \alpha
\end{array}\right]^{\mathrm{T}}
$$

is a symmetric semi-positive definite shape matrix that encodes 
- the ellipse orientation $\alpha$, and

- the semi-axes lengths $l_{1}$ and $l_{2}$.

Approaches for elliptic extended object tracking can be distinguished by the ellipse parameterization they employ. For this reason, the next subsection discusses methods that estimate the shape matrix $X$ itself, and in the subsequent subsection, we present methods that directly estimate the orientation and semi-axes lengths.

\subsection{RANDOM MATRIX APPROACHES}

The random matrix approach [19], [20] estimates the shape matrix (19). For this purpose, the state vector $\mathrm{x}$ in Eq. (3) is decomposed into ${ }^{1}$

- the position and kinematics $\mathbf{m}$,

- and the shape matrix $X$, which implicitly encodes the shape parameters of $\mathbf{p}$ in (3).

The random matrix approach was extensively discussed in our previous overview article [7]. Here, we give a tutorial introduction to the basic approach from [20] and then discuss recent advances and new developments.

\subsubsection{Basic Approach}

The random matrix approach assumes that the measurements are scattered over the entire ellipse surface according to the Gaussian spatial measurement source distribution, i.e.,

$$
p(\mathbf{y} \mid \mathbf{m}, X)=\mathcal{N}(\mathbf{y} ; H \mathbf{m}, z X),
$$

where a measurement source $\mathbf{y}$ is Gaussian distributed with

- mean $H \mathbf{m}$, and

- covariance matrix $z X$ with a scaling factor $z \in \mathbb{R}$.

The scaling factor $z$ in Eq. 20, is usually set to $\frac{1}{4}$ in order to match a uniform spatial measurement source distribution, i.e.,

$$
\mathcal{N}\left(\mathbf{z} ; H \mathbf{m}, \frac{1}{4} X\right) \approx \mathcal{U}(\mathbf{z} ; H \mathbf{m}, X) .
$$

With additional additive Gaussian sensor noise with covariance $R$ according to Eq. (13), we obtain from Eq. (9) the spatial distribution

$$
\begin{aligned}
\phi(\mathbf{z} \mid \mathbf{m}, X) & =\int p(\mathbf{z} \mid \mathbf{y}) \cdot p(\mathbf{y} \mid H \mathbf{m}, X) \mathrm{d} \mathbf{y} \\
& =\mathcal{N}(\mathbf{z} ; H \mathbf{m}, z X+R)
\end{aligned}
$$

of a single measurement $\mathbf{z}$. The spatial density represented by (21) is illustrated in Fig. 8 .

In the random matrix approach, an ellipse estimate for the shape matrix $X$ is captured with an inverse Wishart density [21]

$$
\mathcal{I W}_{d}(X ; v, V)
$$

that has two parameters, namely,

- a symmetric positive definite matrix

$$
V \in \mathbb{R}^{d \times d} \text { (scale matrix) }
$$

\footnotetext{
${ }^{1}$ In the following, we omit the time index $k$ as only a single update step is considered.
}

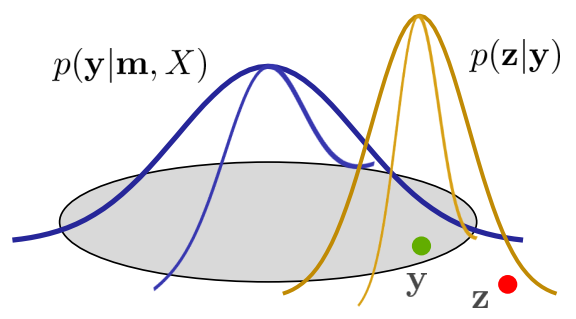

FIGURE 8. Spatial density for the random matrix approaches: The spatial measurement source density is Gaussian with covariance $z X$, and additionally there is additive Gaussian measurement noise.

- and an one-dimensional parameter

$$
v>2 d \text { (degree of freedom). }
$$

The mean of the inverse Wishart density is given by

$$
\frac{1}{v-2 d-2} V
$$

for $v-2 d-2>0$.

For ellipses in two-dimensional space, we would have $d=2$ and in three-dimensional space $d=3$. An advantage of the inverse Wishart density is that it inherently incorporates the constraint that $X$ is a symmetric positive definite matrix, i.e., it always describes a valid ellipse. A disadvantage of this representation, however, is that the uncertainty of the parameters is encoded in a single scalar value, e.g., it does not explicitly distinguish the uncertainties of all three degrees of freedom of an ellipse.

In the factorized random matrix model [20], the estimate for the kinematic state is represented by means of a Gaussian distribution, which is assumed to be independent of the shape estimate. In total, an extended object estimate is represented by the density

$$
p(\mathbf{x}, X)=\mathcal{N}\left(\mathbf{x} ; \mathbf{m}_{-}, P_{-}\right) \times \mathcal{I} \mathcal{W}_{d}\left(X ; v_{-}, V_{-}\right) .
$$

where

- $\mathbf{m}_{-}$is the mean of the kinematic state and $P_{-}$its covariance matrix,

- $V_{-}$is the extent estimate and $v_{-}$its degree of freedom. Based on this representation, the measurement likelihood function Eq. 21], and a given a set of measurements

$$
\mathbf{W}=\left\{\mathbf{z}^{1}, \ldots, \mathbf{z}^{n}\right\}
$$

it is possible to derive a closed-form approximation for the posterior

$$
p(\mathbf{x}, X \mid \mathbf{W}) \approx \mathcal{N}\left(\mathbf{x} ; \mathbf{m}_{+}, P_{+}\right) \times \mathcal{I W}_{d}\left(X ; v_{+}, V_{+}\right),
$$

where

- $\mathbf{m}_{+}$is the posterior mean of the kinematic state and $P_{+}$ its covariance matrix,

- $V_{+}$is the extent estimate and $v_{+}$its degree of freedom. The formulas for the measurement update from [20] are given in Table 2 . 
Essentially, the kinematic state estimate is updated with the Kalman filter formulas, where the uncertainty from the shape is incorporated. The shape update employs, a "pseudomeasurement" by calculating the scattering matrix $Z$ defined as

$$
\begin{aligned}
\overline{\mathbf{z}} & =\frac{1}{n} \sum_{\mathbf{z}^{j} \in \mathbf{W}} \mathbf{z}^{j}, \\
Z & =\sum_{\mathbf{z}^{j} \in \mathbf{W}}\left(\mathbf{z}^{j}-\overline{\mathbf{z}}\right)\left(\mathbf{z}^{j}-\overline{\mathbf{z}}\right)^{\mathrm{T}} .
\end{aligned}
$$

Due its compact closed-form expressions and the applicability to a wide-range of sensor data, the random matrix approach became a widely-used method for extended object tracking. A huge variety of further developments and variations are available in the meantime. In our previous overview article [7], we described different measurement models and measurement update formulas and discussed different approaches for the prediction step.

\subsubsection{Further Extensions, Models and Methods a: Prediction}

Besides of the works [22], [23] that introduce approaches for incorporating the temporal evolution of the extent in the prediction, the work [24] proposes to use the non-central inverse Wishart distribution to model state transitions and to derive closed-form expressions for the prediction. In [25], a robust prediction method for targets performing a constant turn is presented.

\section{b: Nonlinear Measurements}

The original random matrix approach assumes a linear measurement model, i.e., Cartesian measurements according to Eq. (11). For this reason, approaches to deal with nonlinear measurement equations have been developed recently, e.g., [26]-[28]. In [27], statistical linearization and Variational Bayes (VB) are used to deal with nonlinear measurement equations, and the methods [26], [28] are based on converted measurements.

\section{c: Unknown Sensor Noise}

A method to deal with an unknown and possibly time-varying noise covariance is proposed in [29] using VB.

\section{$\mathrm{d}$ : Partial Measurements}

For many sensors such as automotive radar, the target object is only partially visible due to the current sensor-totarget geometry or occlusions. For this reason, the random matrix approach has been extended to deal with partial view measurements by means of a learned hierarchical truncated Gaussian model in [30] and the work [31] is based on transforming an elliptic shape that is represented as random matrix.
TABLE 2. Random matrix update from 20.

Input: Prior density $\sqrt{23}$ ) specified by $\mathbf{m}_{-}, P_{-}$and $v_{-}, V_{-}$, set of detections $\mathbf{W}, n=|\mathbf{W}|$, measurement noise covariance $R$

Output: Posterior density parameterized by $\mathbf{m}_{+}, P_{+}, v_{+}, V_{+}$

$$
\begin{aligned}
\overline{\mathbf{z}} & =\frac{1}{n} \sum_{\mathbf{z}^{i} \in \mathbf{W}} \mathbf{z}^{i} \\
\varepsilon & =\overline{\mathbf{z}}-H \mathbf{m}_{-} \\
Z & =\sum_{\mathbf{z}^{i} \in \mathbf{W}}\left(\mathbf{z}^{i}-\overline{\mathbf{z}}\right)\left(\mathbf{z}^{i}-\overline{\mathbf{z}}\right)^{\mathrm{T}} \\
Y & =z \hat{X}+R \\
S & =H P_{-} H^{\mathrm{T}}+\frac{Y}{n} \\
K & =P_{-} H^{\mathrm{T}} S^{-1} \\
\hat{X} & =V_{-}\left(v_{-}-2 d-2\right)^{-1} \\
\hat{N} & =\hat{X}^{\frac{1}{2}} S^{-\frac{1}{2}} \varepsilon \varepsilon^{\mathrm{T}} S^{-\frac{T}{2}} \hat{X}^{\frac{T}{2}} \\
\hat{Z} & =\hat{X}^{\frac{1}{2}} Y^{-\frac{1}{2}} Z Y^{-\frac{\mathrm{T}}{2}} \hat{X}^{\frac{\mathrm{T}}{2}} \\
\mathbf{m}_{+} & =\mathbf{m}_{-}+K \varepsilon \\
P_{+} & =P_{-}-K S K^{\mathrm{T}} \\
v_{+} & =v_{-}+n \\
V_{+} & =V_{-}+\hat{N}+\hat{Z}
\end{aligned}
$$

\section{e: Smoothing}

In [32], closed-form smoothing formulas for the random matrix approach are derived for both the factorized model 23) but also the original conditional model [7], [19].

\section{f: Classification}

Recent works on joint tracking and classification of extended objects [33]-[37] simultaneously determine the shape of the object and its class.

\section{g: Multisensor Settings}

In [38], object estimates from multiple sensors are fused based on the weighted Kullback-Leibler divergence. In [39], approaches for fusing measurements from multiple sensors in the random matrix framework are investigated, and [40] provides an extension to asynchronous sensors.

\subsection{EXPLICIT PARAMETERIZATION}

Recently, new approaches for elliptical extended object tracking emerged that explicitly parameterize an ellipse with its semi-axes lengths and orientation. As in the example parameterization in Eq. (4), the extent parameters are

$$
\mathbf{p}=\left[\alpha, l_{1}, l_{2}\right]^{T},
$$

where $\alpha$ is the (counter-clockwise) orientation and $l_{1}$ and $l_{2}$ denote the semi-axes lengths. An obvious problem with this parameterization is that it is not unique, i.e., multiple different parameters describe the same ellipse. Furthermore, there are constraints on the parameters, i.e., $\alpha \in[0, \pi), l_{1}>0$ and $l_{2}>0$. An advantage is that the uncertainties of all three ellipse parameters can be explicitly distinguished and motion models can be formulated intuitively. Depending on the specific method, a tight coupling between the kinematic and shape parameters is possible.

In the following, we will describe two approaches for the case that measurements are (uniformly) scattered over the entire target surface, i.e., the MEM-EKF* approach in Section 3.2.1 and the Independent Axes Estimation (IAE) 
approach in Section 3.2.2. The measurement likelihoods of the MEM-EKF* and IAE approach are actually identical to the likelihood Eq. 21] used in the random matrix approaches.

\subsubsection{MEM-EKF*}

Next, we will explain an approach based on the Multiplicative Error Model (MEM) [41]. The key idea is to form a measurement equation that maps the state vector $\mathbf{x}$ (using the parameterization (28) ) to the measurement $\mathbf{z}$ according to

$$
\mathbf{z}=H \mathbf{m}+\underbrace{\operatorname{Rot}(\alpha)\left[\begin{array}{cc}
l_{1} & 0 \\
0 & l_{2}
\end{array}\right]}_{:=S}\left[\begin{array}{l}
h_{1} \\
h_{2}
\end{array}\right]+\mathbf{v},
$$

where

- $\mathbf{h}=\left[h_{1}, h_{2}\right]^{T}$ is multiplicative Gaussian noise with covariance matrix $z \cdot I_{2 \times 2}$ and scaling constant $z \in \mathbb{R}$, and

- $\mathbf{v}$ is additive Gaussian measurement noise with covariance $R$.

The likelihood corresponding to the measurement equation (29) is

$$
p(\mathbf{z} \mid \mathbf{m}, \underbrace{\alpha, l_{1}, l_{2}}_{\mathbf{p}})=\mathcal{N}(\mathbf{z} ; H \mathbf{m}, z \underbrace{S S^{\mathrm{T}}}_{X}+R),
$$

which is identical to Eq. 211 - only the parameterization of the ellipse differs.

Measurement equation (29) can now serve as a basis to derive Kalman filter-based estimators for the state $\mathbf{x}=$ $\left[\mathbf{p}^{\mathrm{T}}, \mathbf{m}^{\mathrm{T}}\right]^{T}$. However, a direct application of general nonlinear Kalman filtering techniques such as the Unscented Kalman filter (UKF) [18] or Extended Kalman filter (EKF) [8] does not yield satisfying results due to the strong nonlinearities and the zero-mean multiplicative noise. For this reason, a problem tailored variant that is based on a pseudomeasurement, the so-called MEM-EKF*, was proposed in [42]. The MEM-EKF* employs an individual measurement $\mathbf{z}$ for updating a prior for the state $\mathrm{x}$ specified by

$$
p^{-}(\mathbf{m}, \mathbf{p})=\mathcal{N}\left(\mathbf{m} ; \mathbf{m}_{-}, C_{-}^{\mathbf{m}}\right) \times \mathcal{N}\left(\mathbf{p} ; \mathbf{p}_{-}, C_{-}^{\mathbf{p}}\right)
$$

where

- $\mathbf{m}_{-}$denotes the estimate for the kinematic state and $\mathbf{p}_{-}$ the estimate for the shape parameters, and

- the matrices $C_{-}^{\mathbf{m}}, C_{-}^{\mathbf{p}}$ are the corresponding covariance matrices of the estimates.

The formulas to calculate the posterior density in the form

$$
p^{+}(\mathbf{m}, \mathbf{p} \mid \mathbf{z}) \approx \mathcal{N}\left(\mathbf{m} ; \mathbf{m}_{+}, C_{+}^{\mathbf{m}}\right) \times \mathcal{N}\left(\mathbf{p} ; \mathbf{p}_{+}, C_{+}^{\mathbf{p}}\right)
$$

are given in Table 3 .

Similar to the random matrix approach, a pseudomeasurement is constructed, but only for a single measurement $\mathbf{z}=\left[\mathbf{z}_{1}, \mathbf{z}_{2}\right]^{T}$, i.e.,

$$
\hat{\mathbf{z}}=\left[\begin{array}{c}
\left(\mathbf{z}_{1}-\overline{\mathbf{z}}_{1}\right)^{2} \\
\left(\mathbf{z}_{2}-\overline{\mathbf{z}}_{2}\right)^{2} \\
\left(\mathbf{z}_{1}-\overline{\mathbf{z}}_{1}\right)\left(\mathbf{z}_{2}-\overline{\mathbf{z}}_{2}\right)
\end{array}\right],
$$

TABLE 3. Measurement update of the MEM-EKF* algorithm 42.

Input: Measurement $\mathbf{z}$, prior estimates $\mathbf{m}_{-}, \mathbf{p}_{-}, C_{-}^{\mathbf{m}}$, and $C_{-}^{\mathbf{p}}$

Output: Updated estimates $\mathbf{m}_{+}, \mathbf{p}_{+}, C_{+}^{\mathbf{m}}$, and $C_{+}^{\mathbf{p}}$

$$
\begin{aligned}
& F=\left[\begin{array}{llll}
1 & 0 & 0 & 0 \\
0 & 0 & 0 & 1 \\
0 & 1 & 0 & 0
\end{array}\right], \widetilde{F}=\left[\begin{array}{llll}
1 & 0 & 0 & 0 \\
0 & 0 & 0 & 1 \\
0 & 0 & 1 & 0
\end{array}\right] \\
& S=\left[\begin{array}{l}
S_{1} \\
S_{2}
\end{array}\right]=\operatorname{Rot}\left(\alpha^{-}\right)\left[\begin{array}{cc}
l_{1}^{-} & 0 \\
0 & l_{2}^{-}
\end{array}\right] \\
& J_{1}=\left[\begin{array}{ccc}
-l_{1}^{-} \sin \alpha^{-} & \cos \alpha^{-} & 0 \\
-l_{2}^{-} \cos \alpha^{-} & 0 & -\sin \alpha^{-}
\end{array}\right] \\
& J_{2}=\left[\begin{array}{ccc}
l_{1}^{-} \cos \alpha^{-} & \sin \alpha^{-} & 0 \\
-l_{2}^{-} \sin \alpha^{-} & 0 & \cos \alpha^{-}
\end{array}\right] \\
& C^{\mathrm{I}}=S C^{\mathbf{h}} S^{\mathrm{T}} \\
& C^{\mathrm{II}}=\left[\epsilon_{m n}\right]=\operatorname{Tr}\left(C_{-}^{\mathbf{p}} J_{n}^{\mathrm{T}} C^{\mathbf{h}} J_{m}\right) \text { for } m, n=1,2 \\
& M=\left[\begin{array}{c}
2 S_{1} C^{\mathbf{h}} J_{1} \\
2 S_{2} C^{\mathbf{h}} J_{2} \\
S_{1} C^{\mathbf{h}} J_{2}+S_{2} C^{\mathbf{h}} J_{1}
\end{array}\right] \\
& C^{\mathbf{m z}}=C_{-}^{\mathbf{m}} H^{\mathrm{T}} \\
& C^{\mathbf{z}}=H C_{-}^{\mathbf{m}} H^{\mathrm{T}}+C^{\mathrm{I}}+C^{\mathrm{II}}+R \\
& \hat{\mathbf{z}}=F\left(\left(\overline{\mathbf{z}}-H \mathbf{m}_{-}\right) \otimes\left(\mathbf{z}-H \mathbf{m}_{-}\right)\right) \\
& \overline{\mathbf{z}}=F \operatorname{vect}\left(C^{\mathbf{z}}\right) \\
& C^{\mathbf{p} \hat{\mathbf{z}}}=C_{-}^{\mathbf{p}} M^{\mathrm{T}} \\
& C^{\hat{\mathbf{z}}}=F\left(C^{\mathbf{z}} \otimes C^{\mathbf{z}}\right)(F+\widetilde{F})^{\mathrm{T}} \\
& \mathbf{m}_{+}=\mathbf{m}_{-}+C^{\mathbf{m z}}\left(C^{\mathbf{z}}\right)^{-1}\left(\mathbf{z}-H \mathbf{m}_{-}\right) \\
& C_{+}^{\mathbf{m}}=C_{-}^{\mathbf{m}}-C^{\mathbf{m z}}\left(C^{\mathbf{z}}\right)^{-1}\left(C^{\mathbf{m z}}\right)^{\mathrm{T}} \\
& \mathbf{p}_{+}=\mathbf{p}_{-}+C^{\mathbf{p} \hat{\mathbf{z}}}\left(C^{\hat{\mathbf{z}}}\right)^{-1}(\hat{\mathbf{z}}-\overline{\mathbf{z}}) \\
& C_{+}^{\mathbf{p}}=C_{-}^{\mathbf{p}}-C^{\mathbf{p} \hat{\mathbf{z}}}\left(C^{\hat{\mathbf{z}}}\right)^{-1}\left(C^{\mathbf{p} \hat{\mathbf{z}}}\right)^{\mathrm{T}}
\end{aligned}
$$

where $\overline{\mathbf{z}}=\left[\overline{\mathbf{z}}_{1}, \overline{\mathbf{z}}_{2}\right]^{T}$ is (for example) the predicted measurement $H \mathbf{m}_{-}$. The pseudo-measurement can be compactly written by means of the 2 -fold Kronecker product $\otimes$, see Table 3

\subsubsection{Independent axes estimation}

The Independent Axes Estimation (IAE) approach [43] is based on measurement equation 29p. It independently estimates the semi-axes $l_{1}$ and $l_{2}$ of the ellipse, where it assumes that the ellipse orientation $\alpha$ is aligned with the target velocity vector, i.e., it is necessary to incorporate the target velocity $\left[v_{x}, v_{y}\right]^{T}$ in the kinematic parameters $\mathbf{m}$. The target orientation is then given by $\alpha=\operatorname{atan} 2\left(v_{y}, v_{x}\right)$.

The IAE approach employs all available measurements $\mathbf{W}$ from a scan. A pseudo-measurement is created based on the normalized spread matrix $\bar{Z}=\frac{1}{n} Z$, where $Z$ is defined in Eq. 27]. The spread matrix $\bar{Z}$ can be written as

$$
\bar{Z}=V \cdot D \cdot V^{\mathrm{T}}
$$

where $D=\left[\begin{array}{cc}d_{1} & 0 \\ 0 & d_{2}\end{array}\right]$ is a diagonal matrix with the pseudomeasurements $d_{1}$ and $d_{2}$, and $V$ a rotation matrix.

Based on independent priors for the kinematic parameters and the semi-axes

$$
\begin{aligned}
p(\mathbf{m}) & \sim \mathcal{N}\left(\mathbf{m} ; \mathbf{m}_{-}, C_{-}^{\mathbf{m}}\right), \\
p\left(l_{i}\right) & \sim \mathcal{N}\left(l_{i} ; l_{-}^{i}, c_{-}^{l_{i}}\right), i=1,2,
\end{aligned}
$$


the IAE approach determines the posterior

$$
\begin{aligned}
p(\mathbf{m} \mid \mathbf{W}) & \sim \mathcal{N}\left(\mathbf{m} ; \mathbf{m}_{+}, C_{+}^{\mathbf{m}}\right), \\
p\left(l_{i} \mid d_{i}\right) & \sim \mathcal{N}\left(l_{i} ; l_{+}^{i}, c_{+}^{l_{i}}\right), i=1,2
\end{aligned}
$$

based on the approximation $\operatorname{Rot}(\alpha) \approx V$.

The IAE update can only be applied if there are more than two measurements in a scan, because otherwise, the scatter matrix $Z$ cannot be calculated. An advantage of the IAE approach is that only a single axis can be updated in case the other one is not visible due to occlusions.

\subsection{FURTHER EXTENSIONS, MODELS AND METHODS}

\section{a: Fixed and known axes-lengths}

A MEM-EKF* version for known extent (i.e., only the orientation is estimated) is presented in [44] and its applicability for maritime RADAR is shown. In [45], the special case of fixed but unknown axes-lengths was treated based on maximum likelihood estimation.

\section{b: Variational Bayes update}

A novel approach that explicitly estimates the orientation and axes length based on the random matrix model was proposed in [46]. It employs Variational Bayes (VB) techniques to derive an iterative measurement update.

\section{c: EM-update}

In [47], an EM-based measurement update is proposed that allows for a tight coupling between extent and kinematic parameters.

\section{d: RHM for ellipses}

The Random Hypersurface Model (RHM) for star-convex shapes, which will be discussed in Section 4.1, can also be used for elliptic shapes [48]. In contrast to the MEM-EKF* and random matrix approaches, it allows for (approximately) modeling both measurement sources that are uniformly distributed on the shape and measurements that are concentrated on the contour. However, the likelihood for an RHM that matches a uniform distribution is not a Gaussian distribution as (21) and (30). A recent comparison of the MEM-EKF* and IAE approach with an RHM for ellipses can be found in [49].

In [50], image moments are used to represent an elliptic shape within the RHM framework and the UKF is used for inference.

\section{e: Lie groups}

In [51], banana-shaped objects (i.e., space debris clusters) are parameterized based on Lie groups and an iterated Extended Kalman Filter (IEKF) is derived for centroid estimation. In [52], an elliptical extended target tracking approach using the affine group is developed based on a measurement model [53], which is based on the generation of predicted measurements on the boundary of an elliptic (or rectangular) target.

\section{f: Volcanormal approach}

In [54], the volcanormal density is used to model radar detections that come rather from the boundary of the target than the inner, and a maximum likelihood estimator is derived.

\section{g: Distributed estimation and shape level fusion}

In the work [55], a diffusion algorithm for distributed extended target tracking based on the MEM-EKF* was developed.

The problem of fusing two elliptic shape estimates that use the explicit representation Eq. (28), has been considered in [56]. The task is to fuse two elliptic shape estimates, i.e.,

- $\mathbf{x}^{-}=\left[\alpha^{-}, l_{1}^{-}, l_{2}^{-},\left(\mathbf{m}^{-}\right)^{\mathrm{T}}\right]^{T}$ with covariance $C^{-}$, and

- $\mathbf{x}^{*}=\left[\alpha^{*}, l_{1}^{*}, l_{2}^{*},\left(\mathbf{m}^{*}\right)^{\mathrm{T}}\right]^{T}$ with covariance $C^{*}$.

A main challenge is that multiple parameters may represent the same ellipse such that a standard linear fusion (i.e., a weighted average) is meaningless. For example, fusing an axis-aligned ellipse given by

$$
\alpha^{-}=0, l_{1}^{-}=5, \text { and } l_{2}^{-}=1
$$

with the ellipse given by

$$
\alpha^{*}=\frac{\pi}{2}, l_{1}^{*}=1, \text { and } l_{2}^{*}=5
$$

results in a circle with radius 3 (given $C^{-}=C^{*}$ ), which is quite counter-intuitive as the two estimates actually specify the same ellipse. In order to incorporate that estimates represent elliptic shapes, the work [56] introduces two concepts:

- A new type of probability density function named Random Ellipse Density (RED), which is tailored to the explicit parameterization, is introduced. A RED is represented by a Gaussian mixture and it allows for systematic fusion of elliptic shape estimates, where the fusion result is represented by a Gaussian mixture, again.

- The so-called Minimum Mean Gaussian Wasserstein (MMGW) estimate is introduced, which denotes the optimal elliptic shape estimate according to the Gaussian Wasserstein distance. The Gaussian Wasserstein distance measures the distance between elliptic shapes, see Section 6.1.

\section{STAR-CONVEX APPROACHES}

In case there is a sufficient amount of measurements available per scan and the target extent is relatively large in comparison to the magnitude of the measurement noise, a more detailed shape description might be reasonable. In particular starconvex shape models turned out to be suitable for many real-world applications (such as LIDAR data for automotive environment perception) as they can represent the underlying target geometry precisely, while still being computationally tractable. For a star-convex extended object, the assumption is that the line segment from the target center $H \mathbf{m}$ to any point on the contour is completely on the target surface. A star-convex object extent can be represented by a radial function

$$
r_{\mathbf{p}}(\phi):[0,2 \pi) \rightarrow \mathbb{R}^{+}
$$




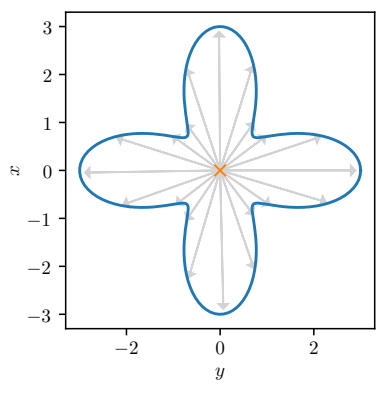

(a) Star-convex shape

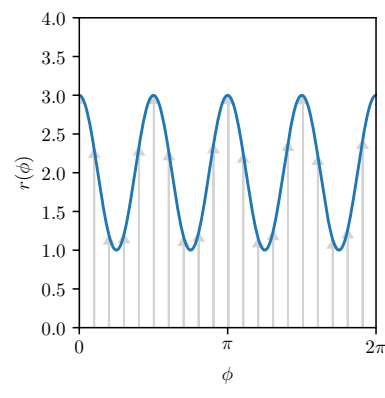

(b) Radial function
FIGURE 9. A star-convex shape (left) and its representation as a radial function (right).

which is parameterized by $\mathbf{p}$, and specifies the distance from the center $H \mathbf{m}$ to a measurement source depending on an angle $\phi$, see Fig. 9 b for an illustration.

\subsection{RANDOM HYPERSURFACE MODEL}

Star-convex extended objects have been initially introduced using the Random Hypersurface Model (RHM) [48] for modeling measurement sources on the extent. The key insight is that it is usually possible to formulate a measurement equation in case the measurement sources are on the boundary of the object (i.e., on a hypersurface). By means of a random scaling factor, the measurement equation for the boundary can be scaled to cover the entire star-convex surface. This procedure can mathematically be interpreted as homotopy. The RHM can be formulated with explicit or implicit measurement equations, which will be discussed in the following.

\subsubsection{Explicit Measurement Equation}

The explicit measurement equation for star-convex extended objects [48] maps

- the center $H \mathbf{m}$,

- the shape parameters $\mathbf{p}$,

- an unknown scaling factor $s$,

- additive sensor noise $\mathbf{v}$, and an

- unknown angle $\phi$

to an individual measurement $\mathbf{z}$ according to

$$
\mathbf{z}=s \cdot \underbrace{\left[\begin{array}{c}
\cos (\phi) \\
\sin (\phi)
\end{array}\right]}_{:=\mathbf{u}(\phi)} \cdot r_{\mathbf{p}}(\phi)+H \mathbf{m}+\mathbf{v} .
$$

The unknown scaling factor $s$ is usually modeled as multiplicative noise. Actually, it can be shown that in case the measurement sources are uniformly distributed on the target, the mean of $s$ is $\hat{s}=0.8$ and its variance $\sigma_{s}^{2}=\frac{1}{12}$. The angle $\phi$ specifies the direction of the measurement source to the center, relatively to the target orientation $\alpha$. If $\phi$ would be known, Eq. 37 would be a measurement equation with both additive and multiplicative noise. Three possible approaches for dealing with the unknown angle $\phi$ have been considered.

\section{- Greedy Association Model (GAM)}

The greedy association [48], [57] employs the measurement $\mathbf{z}$ and target state to determine an estimate for $\phi$, e.g.,

$$
\phi \approx \angle(\mathbf{z}-H \mathbf{m}, \mathbf{u}(0))
$$

Based on the greedy approach and Eq. (37), an EKF or UKF can be employed for the measurement update. However, the GAM is a rough approximation that only gives satisfying results in case of relatively low measurement and system noise. For high measurement noise it leads to a bias in the extent estimate and for high system noise the shape estimate might even diverge.

- Probability distribution for angle

A more realistic modeling is to assume that the angle $\phi$ is distributed according to a probability density function $p(\theta)$, e.g., a uniform distribution between 0 and $2 \pi$. However, due to the nonlinear influence of $\phi$ in Eq. 37, it becomes challenging to derive a closed-form update. Nevertheless, an efficient closed-form iterative measurement update based on Expectation-Maximization (EM) has been proposed in [58], [59] and [60].

\section{- Partial likelihood}

Already in the initial works about star-convex shape estimation [48], it was proposed to improve the greedy association by means of reformulating Eq. 37. to a onedimensional equation

$$
\begin{aligned}
0=s^{2} \cdot\left\|r_{\mathbf{p}}(\phi)\right\|^{2} & +2 \cdot s \cdot r_{\mathbf{p}}(\phi) \cdot \mathbf{u}(\phi)^{\mathrm{T}} \cdot \mathbf{v} \\
& +\|\mathbf{z}-H \mathbf{m}\|^{2}+\|\mathbf{v}\|^{2},
\end{aligned}
$$

which essentially works with the squared distance of the measurement to the center. For the remaining $\phi$ in Eq. (39), the greedy approach can be used. The benefit of this "projection" is that the influence of the unknown $\phi$ is reduced. This concept has been further investigated in the general context of partial likelihoods in [57]. The implicit measurement equation avoids a bias in the shape estimate (when using a nonlinear Kalman filter) but due to the projection of the measurement to a scalar, information is lost and further nonlinearities are introduced.

Various parameterizations for the radial function have been proposed in the context of star-convex extended object tracking such as Fourier coefficients [48] and Gaussian processes [61]. While the initial works were based on Fourier coefficients, most recent works employ a Gaussian process representation, which will be introduced in Section 4.2.

Note that it is possible to explicitly incorporate the orientation $\alpha$ in the shape parameters. In this case it is suitable to define $r_{\mathbf{p}}(\phi):=r_{\mathbf{p}}^{l}(\phi-\alpha)$, where $r^{l}$ is the radial function in local coordinates.

\subsubsection{Implicit measurement equation}

Initially, the RHM was introduced based on implicit measurement equations. Several star-convex contours such as circles 

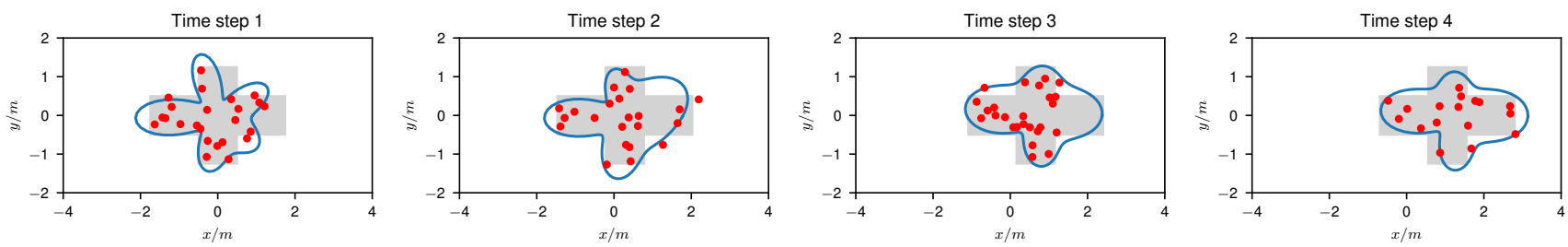

FIGURE 10. Example run of the Simple-GP-RHM algorithm: Depicted are the measurements (red dots), the cross-shaped extended object (gray), and the shape estimates (blue) for the first four time steps.

or ellipses can be described by an implicit equation in the form

$$
g(H \mathbf{m}, \mathbf{p}, \mathbf{y})=0,
$$

where $H \mathbf{m}$ is the center and $\mathbf{p}$ is the shape vector. For example, for a circle, the implicit equation is

$$
\|H \mathbf{m}-\mathbf{y}\|^{2}-r^{2}=0,
$$

where the shape parameter $\mathbf{p}$ only consists of the radius $r$. In order to describe the surface of the star-convex shape, one can add a scalar noise parameter $s \in[0,1]$ that randomly scales the contour, i.e., we obtain an implicit equation in the form

$$
g^{*}(\mathbf{d}, \mathbf{p}, \mathbf{y}, s)=0 \text {. }
$$

For a circle, the implicit equation becomes

$$
\|H \mathbf{m}-\mathbf{y}\|^{2}-(s \cdot r)^{2}=0 .
$$

Together with the sensor model specified by Eq. (12), i.e.,

$$
\mathbf{z}=\mathbf{y}+\mathbf{v},
$$

the implicit RHM in Eq. (41) forms the measurement model for an individual measurement $\mathbf{z}$. It is important to note, that the only assumption that is imposed on the measurement source $\mathbf{y}$ is that it fulfills Eq. (41). The implicit representation reduces the shape estimation problem to curve fitting problems, see for example, [62], [63]. In order to deal with the unknown measurement source, the same options as for the angle $\phi$ in the explicit measurement equation (37) have been investigated, i.e., the best (nearest) measurement source can be used, a probability density can be assumed, or a partial likelihood can be formed [57]. In order to use a Kalman filter for sequential estimation, it is common to linearize the implicit equation (41). For more details, see the discussions in [64]. For example, in [65], an implicit RHM based on level sets has been developed for tracking arbitrary shaped objects modeled with polygons.

\subsection{GAUSSIAN PROCESS APPROACH}

The Gaussian process approach for star-convex shapes introduced in [61] is based on the explicit measurement equation for a random hypersurface model given by Eq. (37). In the following, we will introduce a simplified version of [61], which, among others, does not explicitly incorporate the target orientation, and decorrelates the kinematic and shape parameters.
The radial function $r(\phi)$ in Eq. 36] is represented with a Gaussian process [66]. For this purpose, the function values $r\left(\phi_{1}\right), \ldots, r\left(\phi_{n}\right)$ at $n$ basis points $\phi_{1}, \ldots, \phi_{n}$ are used. The function values are assumed to be jointly Gaussian, where the prior distribution is zero-mean according to

$$
\mathbf{p}=\left[\begin{array}{c}
r\left(\phi_{1}\right) \\
\vdots \\
r\left(\phi_{n}\right)
\end{array}\right] \sim \mathcal{N}\left(\mathbf{p} ; \mathbf{0}, K^{p}\right)
$$

with

$$
K^{p}=\left[\begin{array}{ccc}
k\left(\phi_{1}, \phi_{1}\right) & \ldots & k\left(\phi_{1}, \phi_{n}\right) \\
\vdots & & \vdots \\
k\left(\phi_{n}, \phi_{1}\right) & \ldots & k\left(\phi_{n}, \phi_{n}\right)
\end{array}\right]
$$

where the kernel $k\left(\phi, \phi^{\prime}\right)$ encodes the correlation between the function values. For example, a periodic kernel that incorporates the $2 \pi$ periodicity of the radial function can be chosen according to

$$
k\left(\phi, \phi^{\prime}\right)=\sigma_{\mathrm{ker}}^{2} \cdot \exp \left(-\frac{2 \cdot \sin ^{2}\left(\frac{1}{2}\left|\phi-\phi^{\prime}\right|\right)}{l_{\mathrm{ker}}^{2}}\right)+\sigma_{r}^{2}
$$

with variances $\sigma_{\mathrm{ker}}^{2}, \sigma_{r}^{2}$, and kernel width $l_{\mathrm{ker}}$. The variance $\sigma_{r}^{2}$ models that the radial function is a priori a constant $r$ with $r \sim \mathcal{N}\left(r ; 0, \sigma_{r}^{2}\right)$.

For any $\phi$, the basis points $\mathbf{p}$ and $r(\phi)$ are jointly Gaussian, i.e.,

$$
\left[\begin{array}{c}
r(\phi) \\
\mathbf{p}
\end{array}\right] \sim \mathcal{N}\left(\left[\begin{array}{c}
r(\phi) \\
\mathbf{p}
\end{array}\right] ; \mathbf{0},\left[\begin{array}{cc}
k(\phi, \phi) & K(\phi) \\
K(\phi)^{\mathrm{T}} & K^{p}
\end{array}\right]\right)
$$

with

$$
K(\phi)=\left[\begin{array}{lll}
k\left(\phi, \phi_{1}\right) & \ldots & k\left(\phi, \phi_{n}\right)
\end{array}\right] .
$$

For a given $\mathbf{p}$, we can now derive the conditional density $p(r(\phi) \mid \mathbf{p})$, see [66], which yields the relationship

$$
r_{\mathbf{p}}(\phi)=A(\phi) \cdot \mathbf{p}+e
$$

with

$$
\begin{aligned}
A(\phi) & =K(\phi) \cdot\left(K^{p}\right)^{-1}, \\
C^{e} & =k(\phi, \phi)-K(\phi) \cdot\left(K^{p}\right)^{-1} \cdot K(\phi)^{\mathrm{T}} .
\end{aligned}
$$


TABLE 4. Measurement update of the Simple-GP-RHM (derived from 61]).

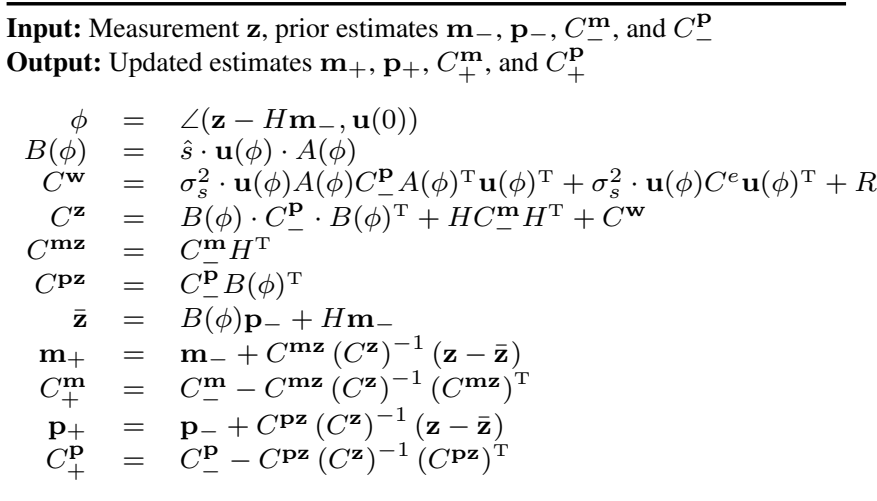

Based on Eq. (47) and Eq. (37), we can then construct the measurement equation

$$
\begin{aligned}
\mathbf{z}= & s \cdot \mathbf{u}(\phi) \cdot(A(\phi) \cdot \mathbf{p}+e)+H \mathbf{m}+\mathbf{v} \\
= & s \cdot \mathbf{u}(\phi) \cdot A(\phi) \cdot \mathbf{p}+H \mathbf{m} \\
& +s \cdot \mathbf{u}(\phi) \cdot e+\mathbf{v} \\
= & \hat{s} \cdot \mathbf{u}(\phi) \cdot A(\phi) \cdot \mathbf{p}+H \mathbf{m}+\mathbf{w}
\end{aligned}
$$

with zero-mean noise

$$
\begin{aligned}
\mathbf{w}:= & (s-\hat{s}) \cdot \mathbf{u}(\phi) \cdot A(\phi) \cdot \mathbf{p} \\
& +s \cdot \mathbf{u}(\phi) \cdot e+\mathbf{v},
\end{aligned}
$$

which is composed of three uncorrelated terms.

For given $\phi$, this is a linear measurement equation, i.e., a Kalman filter can be used for a measurement update if, for example, $\phi$ is approximated with a point estimate. Pseudocode for the measurement update step of the simplified Gaussian process model, called Simple-GP-RHM algorithm, is presented in Table 4 . Fig. 10 illustrates an example run of the Simple-GP-RHM algorithm.

\subsection{CARTESIAN-DEFINED STAR-CONVEX MODELS}

There are scenarios, such as the tracking of vehicles with automotive LIDAR, where

- the sensor resolution allows to resolve a detailed shape contour, and

- the shape of the target is known (e.g., for vehicles), but

- the orientation, length, and width shall be determined.

In this case, it turned out that the description of the target contour by means of a radial function is unsuitable. For example, for an elongated object with relatively low width but a high length, the radial function contains peaks that are difficult to capture with, e.g., Gaussian processes that are based on equidistant basis points. For this reason, [67] proposes to define a star-convex shape directly in Cartesian space using B-splines. Any closed curve, i.e., contour, can be specified in parametric form with a function

$$
C(\tau) \in \mathbb{R}^{2}
$$

that maps a walk parameter $\tau \in[0,1]$ to a two-dimensional point on the contour. As we assume that the star-convex contour $C(\tau)$ of the target is known, the objective is to estimate the orientation $\alpha$, length $l_{1}$, and width $l_{2}$ using the parameterization in Eq. (28). For this purpose, a measurement equation can be formulated according to

$$
\mathbf{z}=s \cdot \operatorname{Rot}(\alpha) \cdot\left[\begin{array}{cc}
l_{1} & 0 \\
0 & l_{2}
\end{array}\right] \cdot C(\tau)+H \mathbf{m}+\mathbf{v}
$$

where $\tau \in[0,1]$ is the walk parameter, and $s \in[0,1]$ is a random scaling factor to capture the target surface. The walk parameter $\tau$ in Eq. 56 is unknown, but similar techniques as for the unknown angle $\phi$ in the radial function in Eq. 37 can be used to deal with it and an EKF can be derived for the measurement update, see [67] for further details.

\subsection{FURTHER EXTENSIONS, MODELS AND METHODS}

\section{a: 3D shapes}

The Gaussian process approach has been extended to threedimensional shapes in [68], [69].

In [70], Non-Uniform Rational B-Splines (NURBS) in three-dimensional Cartesian space are used to model threedimensional extended targets with an implicit measurement equation based on the level-set approach [65]. In [71], spherical double Fourier series are used for describing threedimensional shapes.

\section{b: Improved association}

In [72], an improved method for associating measurements to the star-convex contour is introduced, where the entire measurements from a scan are associated in a batch, instead of sequentially.

\section{c: Improved update}

Recently, a novel iterative measurement update for the Gaussian process approach based on VB has been proposed in [73], which improves over the standard EKF implementation [61].

In [74], a star-convex model is proposed that employs Wishart distributions to ensure that only positive real values are possible for the radial functions.

\section{d: Extension deformation approach}

The extension deformation approach proposed in [75] employs a reference shape such as an ellipse that is deformed to get more complex shapes. For this purpose, control points on the reference shape are defined. By means of shifting the control points, a deformation is achieved with the help of a deformation function. A method for determining point estimates for the measurement sources is developed. With this approach, even non-star-convex shapes can be estimated.

\section{e: Additional features}

Doppler measurements (such as provided by automotive radar) have been incorporated in the Gaussian process approach for star-convex shapes in [76]. In [77], amplitude information is used as an additional feature for Gaussian process based extended target tracking. 


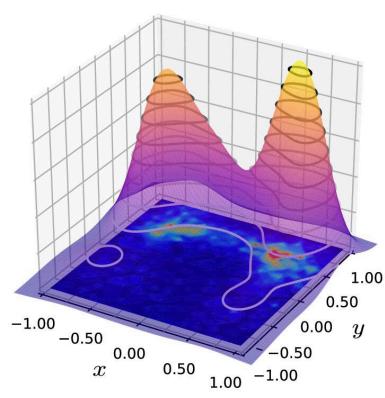

(a)

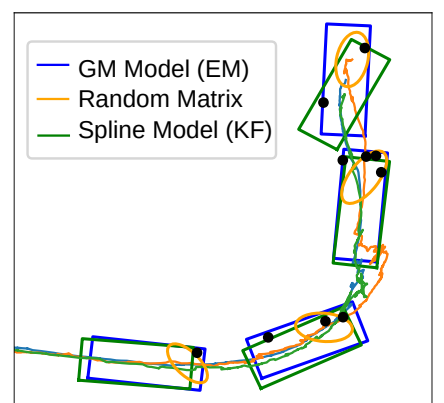

(b)
FIGURE 11. (a) Learned spatial distribution based on the radar data set from the NuScenes benchmark [14] for the case the sensor is in the in front of the vehicle on the left side. (b) Tracking example showing the random matrix approach, the spline model (using Kalman filter) and the Gaussian mixture model (using EM). (Source: adapted from 85])

\section{f: Spatio-temporal Gaussian processes}

Spatio-temporal Gaussian processes [78], which extend standard Gaussian processes with a time component, have been used in [79] to derive an EKF update and a Rauch-TungStreibel Smoother (RTSS).

\section{g: Classification}

In [80], features (such as area) are extracted from Gaussian processes contours and fed into a neural network for classification.

\section{h: Negative information}

Many sensors not only provide measurements that are known to come from the target but they also provide information about where the target is not. For example, the position of a LIDAR detection implies that there is free space between the sensor and the detection. This type of so-called negative information has been incorporated in the Gaussian process approach in [81]. Furthermore, the works [82], [83] introduce methods for incorporating detections that are known to stem not from targets.

\section{i: Multisensor Update}

In [84], a method for merging Gaussian mixture components that represent star-convex extended objects has been developed. The approach is tailored to multisensor applications where the reference point, i.e., the object center, differs due to heterogeneous sensors or a varying sensor-to-object geometry.

\section{GAUSSIAN MIXTURE-BASED APPROACHES}

The approaches for shape estimation that we discussed in the previous sections aim at estimating the parameters of a geometric shape, e.g., an ellipse, where the spatial distribution of the measurements on the target is given. For various target types and sensors, it is appropriate to assume that the measurement sources are uniformly distributed over the target surface, i.e., the spatial distribution is a uniform distribution. However, for more complex scattering patterns of measurement sources, it is difficult to manually specify a reasonable spatial distribution. For this reason, data-driven approaches that learn the spatial distribution by means of real-world sensor data have been developed recently. For this purpose, it is common to employ Gaussian mixtures in order to represent the spatial measurement distribution [86].

\subsection{GAUSSIAN MIXTURE EXTENT MODEL}

In the Gaussian mixture extent model from [86], the target state consists of the length, width and orientation of the target as in Eq. 28). A Gaussian mixture representation of the spatial distribution (9) is learned from real data for a normalized target that is located at the origin, axis aligned, and has unit width and length, i.e., the spatial distribution

$$
\phi\left(\mathbf{z}^{\mathrm{norm}} \mid \mathbf{x}^{\mathrm{norm}}\right)=\sum_{i=1}^{M} \pi_{i} \mathcal{N}\left(\mathbf{z}^{\mathrm{norm}} ; \mu_{i}^{\text {norm }}, \Gamma_{i}^{\text {norm }}\right)
$$

is learned, where $\mathrm{x}^{\mathrm{norm}}$ is the state of the normalized target. See for example [87] for suitable methods for learning Gaussian mixtures from data.

A measurement $\mathbf{z}$ from an arbitrary target with orientation $\alpha$, length $l_{1}$, and width $l_{2}$ is then related to the measurement from the normalized target according to

$$
\mathbf{z}=\boldsymbol{\operatorname { R o t }}(\alpha) \cdot L \cdot \mathbf{z}^{\text {norm }}+H \mathbf{m}
$$

with

$$
L=\left[\begin{array}{cc}
l_{1} & 0 \\
0 & l_{2}
\end{array}\right] .
$$

Hence, the spatial distribution for an arbitrary target becomes

$$
\phi(\mathbf{z} \mid \mathbf{x})=\sum_{i=1}^{M} \pi_{i} \mathcal{N}\left(\mathbf{z} ; \mu_{i}(\mathbf{x}), \Gamma_{i}(\mathbf{x})\right),
$$

where

$$
\begin{aligned}
& \mu_{i}(\mathbf{x})=\boldsymbol{\operatorname { R o t }}(\alpha) \cdot L \cdot \mu_{i}^{\text {norm }}+H \mathbf{m}, \\
& \Gamma_{i}(\mathbf{x})=\boldsymbol{\operatorname { R o t }}(\alpha) \cdot L \cdot \Gamma_{i}^{\text {norm }} \cdot(\boldsymbol{\operatorname { R o t }}(\alpha) \cdot L)^{\mathrm{T}} .
\end{aligned}
$$

As the spatial measurement distribution typically highly depends on the object-to-sensor geometry, [86] employ 8 different spatial distribution models, depending on the angle between the target object and the sensor. By this means it is incorporated that the sensor can only see at most two sides of a vehicle. An example spatial distribution from [85] that is learned from the NuScenes benchmark data set [14] for the case the sensor is in front of the vehicle on the left side, is depicted in Fig.11a

\subsection{MEASUREMENT UPDATE}

For a measurement update based on the likelihood (60), a particle filter has been used in [86]. The work [85] employs Expectation-Maximization (EM) for an iterative solution and [88] proposes an RFS-based update using cluster processes.

Fig. $11 \mathrm{~b}$ depicts the results of the EM-based tracker proposed in [85], the Cartesian-defined spline model [67], and 
the random matrix approach [20]. As the measurements in the scene are concentrated mainly in the front of the vehicle, the random matrix model does not fit the data well, i.e., it tracks the measurement centroid. The Cartesian-defined spline model [67] captures measurements from the boundary, however, it does not incorporate the multi-modal aspects of the spatial distribution. Hence, it struggles in strong maneuvers.

\subsection{FURTHER EXTENSIONS, MODELS AND METHODS}

Apart from the above Gaussian mixture model, further approaches to capture general spatial distributions have been developed. In [89], a B-spline chained ellipse model is presented whose parameters are learned offline, and in [90], an online approach for parameter learning is introduced. Furthermore, in [91], measurements are modeled to come from different regions on the target, where the region probabilities are updated online. The work [31] suggests a Gaussian mixture model that results from transforming an elliptic shape that is represented as a random matrix, however, the parameters are not learned.

\section{METRICS FOR COMPARING EXTENT ESTIMATES}

In order to assess the performance of a target tracker, a metric is required that determines how well the estimate coincides with the ground truth, i.e., the estimation error must be defined. For point target trackers, it is common to employ the (squared) Euclidean distance for calculating the estimation error. However, in case of spatially extended targets, the state vector also contains shape parameters, and the Euclidean distance between shape parameters is - in general - not meaningful. For example, the squared Euclidean distance between the identical ellipses specified by Eq. (34) and Eq. (35) would be larger than zero, i.e.,

$$
\left(0-\frac{\pi}{2}\right)^{2}+(5-1)^{2}+(1-5)^{2}=\frac{\pi^{2}}{4}+32>0,
$$

which is counter-intuitive.

In order to circumvent this problem, (multiple) scalar object features can be determined and the individual errors for the features can be calculated, e.g., an error for the center, an area error, an orientation error etc. Although this approach can give helpful insights on the performance, it does not allow to systematically determine a scalar overall score, e.g., for ranking algorithms. For this purpose, it is necessary to employ a distance metric that accounts for the complete spatial extent of the targets. In particular, Wasserstein [92] and Hausdorff metrics [93] can serve as a basis for defining reasonable metrics. In the following, we will discuss metrics that are commonly used for performance evaluation of elliptic and star-convex shapes.

\subsection{ELLIPTIC SHAPES}

For comparing elliptic extended objects, it was proposed in [94] to use the Wasserstein distance for Gaussian densities. Given two elliptic extended targets with
- center $\mathbf{d}_{1}$ and shape matrix $X_{1}$ for the first target, and

- center $\mathbf{d}_{2}$ and shape matrix $X_{2}$ for the second target,

the so-called squared Gaussian Wasserstein (GW) distance is defined as

$$
\begin{aligned}
& d\left(\mathbf{d}_{1}, X_{1}, \mathbf{d}_{2}, X_{2}\right)^{2}=\left\|\mathbf{d}_{1}-\mathbf{d}_{2}\right\|^{2}+ \\
& \operatorname{Tr}\left(X_{1}+X_{2}-2 \sqrt{\sqrt{X_{1}} X_{2} \sqrt{X_{1}}}\right),
\end{aligned}
$$

where in the above equation, the matrix square root $\sqrt{X}$ of the matrix $X$ fulfills $X=\sqrt{X}^{\mathrm{T}} \sqrt{X}$. The GW distance is used in several works for evaluation of elliptic extended object trackers, e.g., in [24], [27], [43]. Recently, optimal estimators for shape fusion [56] were derived based on the GW distance, see Section 3.3.0g

\subsection{STAR-CONVEX SHAPES}

For star-convex shapes, the Wasserstein distance and Hausdorff distance are computationally expensive to evaluate. For this reason, it was suggested to discretize the shapes [94], [95]. For example, a star-convex shape can be discretized by using $n$ equally spaced angles $\phi_{1}, \ldots, \phi_{n}$ for the radial function [39] to generate $n$-points on the contour [95]. For discrete point sets both the Wasserstein distance and Hausdorff distance can be evaluated more efficiently as it involves an optimal assignment problem that can be solved, e.g., using the Hungarian algorithm [96]-[99].

Furthermore, traditional performance scores from computer vision can be employed for comparing star-convex shapes. For example, in [79], shape mean and shape precision which are discussed in [100] have been used. Say $G$ denotes the true star-convex shape and $S$ its estimate, then the shape recall is defined as the proportion of the area of $G$ that overlaps with $S$, i.e.,

$$
\mathrm{R}(G, S)=\frac{\operatorname{Area}(G \cap S)}{\operatorname{Area}(G)},
$$

and the shape precision is defined as the proportion of the area of $S$ that overlaps with $G$, i.e.,

$$
\mathrm{P}(G, S)=\frac{\operatorname{Area}(G \cap S)}{\operatorname{Area}(S)} .
$$

The Intersection-Over-Union (IoU) score [101] is also commonly used, e.g., in [53], [61], [102], and defined as

$$
\operatorname{IoU}(G, S)=\frac{\operatorname{Area}(G \cap S)}{\operatorname{Area}(G \cup S)} .
$$

However, it must be noted that the above three scores 65, (66), and 67) do not explicitly account for the object center, e.g., if there is no overlap between the estimate and ground truth, the above scores are always 0 . Furthermore, there are no closed-form solutions available for star-convex shapes. 


\section{MULTIPLE EXTENDED OBJECT TRACKING: DESCRIPTION AND MODELING}

Models and algorithms for tracking a single extended object were treated in the first part of this tutorial. In the following sections, we switch focus to tracking multiple extended objects, i.e., we consider the problems of data association and track management.

Consider again Fig. 3, where at time $k$ the measurement set $\mathbf{Z}_{k}$ consists of four detections and there are two target objects. The measurements in the measurement set $\mathbf{Z}_{k}$ are unlabeled, i.e., it is unknown to which target a detection belongs (or if it is clutter). Hence, in order to track multiple extended objects, we need to deal with an unknown measurement origin. Furthermore, the number of objects in the scene is also unknown and might vary over time, i.e., we need to deal with track initialization and termination.

Next, we review Random Finite Set (RFS) theory, which is often used to model мот. Then, we review the standard models for MEOT, both the dynamics model and the measurements model, and lastly we provide a problem definition in this section.

\subsection{RANDOM FINITE SETS}

Random Finite Set (RFS) theory is a mathematical framework for modeling unlabeled and unordered random quantities. It is especially suitable to model uncertainties in MOT, see [9], [10] for a thorough introduction to RFSs. In the following, we present a brief review of RFS theory, specifically the Poisson Point Process (PPP), independent and identically distributed (iid) cluster process, as well as the Bernoulli, multi-Bernoulli (MB) and multi-Bernoulli mixture (MBM) processes.

\subsubsection{Poisson point process}

A PPP is a special RFS with the following two properties:

1) The cardinality of a PPP is Poisson distributed.

2) The elements of a PPP are independent and identically distributed (iid).

A PPP can be represented by an intensity function

$$
\lambda(\mathbf{x}),
$$

which is a function on the single object state space. The intensity function can be decomposed into two parts, i.e.,

$$
\lambda(\mathbf{x})=\mu \cdot f(\mathbf{x}),
$$

where $\mu \geq 0$ denotes a scalar Poisson rate and $f(\mathbf{x})$ denotes a spatial probability distribution.

It is often convenient to represent the intensity function as a mixture of probability density functions according to

$$
\lambda(\mathbf{x})=\sum_{i \in \mathbb{I}} w^{i} p^{i}(\mathbf{x}),
$$

where $\mathbb{I}$ is an index set, $w^{i}>0$ is the weight of the $i$ th component, and $p^{i}(\cdot)$ is the pdf of the $i$ th component. The sum of the weights is non-negative, i.e., $\sum_{i \in \mathbb{I}} w^{i} \geq 0$. The expected number of objects represented by the $i$ th component is given by its weight $w^{i}$; note that this may be larger than one. For a mixture representation of the intensity, the PPP is completely defined by the set of parameters

$$
\left\{\left(w^{i}, p^{i}(\cdot)\right)\right\}_{i \in \mathbb{I}} \text {. }
$$

An important property of the PPP intensity is that for a region $S$ of the state space, the expected number of set members located in $S$ is given by

$$
\int_{\mathbf{x} \in S} \lambda(\mathbf{x}) \mathrm{d} \mathbf{x}
$$

This property can be intuitively interpreted as follows: In regions of the state space with high (resp. low) intensity $\lambda(\mathbf{x})$, there is a high (resp. low) probability that set elements are located. Mathematically, the PPP density is defined as

$$
f(\mathbf{X})=e^{-\mu} \mu^{|\mathbf{X}|} \prod_{\mathbf{x} \in \mathbf{X}} f(\mathbf{x}) .
$$

In MOT, PPPs are the standard model for the clutter measurements and the object birth. In extended object tracking, the most common model for object measurements is a PPP. Lastly, in some tracking filters a PPP is used to model the presence of objects in the surveillance area that have not been detected, see, e.g., [103]-[110].

\subsubsection{Independent identically distributed cluster process}

An iid cluster process is an RFS with the following two properties:

1) The cardinality of an iid cluster process is distributed according to some probability mass function (pmf).

2) The set elements of an iid cluster process are iid. The iid cluster process density is defined as

$$
f(\mathbf{X})=n ! P(n) \prod_{\mathbf{x} \in \mathbf{X}} p(\mathbf{x}),
$$

where $n=|\mathbf{X}|$ denotes the cardinality of $|\mathbf{X}|, P(n)$ is the cardinality pmf, and $p(\mathbf{x})$ is the single object pdf (all set members $\mathbf{x} \in \mathbf{X}$ are distributed according to this pdf). Note that the PPP introduced in Section 7.1.1 is a special case of an iid cluster process, where the cardinality pmf is a Poisson pmf.

The probability hypothesis density (PHD) of an iid cluster process is

$$
D(\mathbf{x})=p(\mathbf{x}) \sum_{n=0}^{\infty} n P(n) .
$$

The PHD function is the first order moment of the multiobject state. Intuitively, the relationship of the PHD to an RFS is similar to the relationship of the expected value to a random variable, see [9].

The iid cluster process is commonly parameterized by the pmf $P(n)$ and the PHD $D(\mathbf{x})$, where the pmf typically is truncated at some maximum cardinality, and the PHD typically has a density mixture representation

$$
D(\mathbf{x})=\sum_{i \in \mathbb{I}} w^{i} p^{i}(\mathbf{x}) .
$$


With this parameterization, the iid cluster process density is defined entirely by the pmf and the set of parameters

$$
P(n),\left\{\left(w^{i}, p^{i}(\cdot)\right)\right\}_{i \in \mathbb{I}} .
$$

In MOT, iid cluster processes are used in the CPHD filter as for example in [111].

\subsubsection{Bernoulli}

A Bernoulli RFS $\mathbf{X}$ is an RFS with the following two properties:

1) With probability $1-r$, a Bernoulli RFS is empty.

2) With probability $r$, a Bernoulli RFS contains a single element with density $f(\mathbf{x})$.

The name Bernoulli RFS derives from the fact that the cardinality is Bernoulli distributed with parameter $r \in[0,1]$. The density of a Bernoulli RFS is defined as

$$
f(\mathbf{X})=\left\{\begin{array}{ll}
1-r & \mathbf{X}=\emptyset \\
r f(\mathbf{x}) & \mathbf{X}=\{\mathbf{x}\} \\
0 & |\mathbf{X}| \geq 2
\end{array} .\right.
$$

A Bernoulli density is completely specified by the tuple

$$
(r, f(\cdot)) \text {. }
$$

In MOT, for each potential object we have uncertainty regarding whether or not there is an actual object there, and if there is an object, we have uncertainty regarding the object state. Hence, a Bernoulli RFS is a natural representation of an object, because it incorporates both the uncertainty of the object's existence (with the probability of existence $r$ ), and the uncertainty of the object's state $\mathbf{x}$ (with the object state density function $f(\mathbf{x})$ ).

\subsubsection{Multi-Bernoulli}

Let $\mathbb{I}$ be an index set for independent Bernoulli RFSs $\mathbf{X}^{i}, i \in$ $\mathbb{I}$, where $\mathbf{X}^{i} \cap \mathbf{X}^{j}=\emptyset$ for all $i, j \in \mathbb{I}$. A multi-Bernoulli (MB) RFS

$$
\mathbf{X}=\cup_{i \in \mathbb{I}} \mathbf{X}^{i}
$$

is the union of independent Bernoulli RFSs. The MB density then becomes

$$
f(\mathbf{X})=\left\{\begin{array}{lll}
\sum_{\uplus_{i \in \mathbb{I}} \mathbf{X}^{i}=\mathbf{X}} \prod_{i \in \mathbb{I}} f^{i}\left(\mathbf{X}^{i}\right) & \text { if } & |\mathbf{X}| \leq|\mathbb{I}| \\
0 & \text { if } & |\mathbf{X}|>|\mathbb{I}|
\end{array},,\right.
$$

where the notation $\uplus$ is introduced in Table 11 The MB distribution is completely defined by the set of Bernoulli parameters

$$
\left\{\left(r^{i}, f^{i}(\cdot)\right)\right\}_{i \in \mathbb{I}} .
$$

In MOT, an MB is a perfectly suitable representation of the potential multiple objects in the surveillance area, because, as mentioned above, each Bernoulli captures the relevant uncertainties for a single object.

\subsubsection{Multi-Bernoulli mixture}

A (normalized) weighted sum of MB RFS densities

$$
f(\mathbf{X})=\sum_{j \in \mathbb{J}} w^{j} f^{j}(\mathbf{X}),
$$

where $f^{j}(\cdot)$ denotes an MB density (also called component) and $w^{j}>0$ denotes a weight, is called a multi-Bernoulli mixture (MBM) density. Note that $\sum_{j \in \mathbb{J}} w^{j}=1$. An MBM is completely defined by the set of parameters

$$
\left\{\left(w^{j},\left\{\left(r^{j, i}, f^{j, i}(\cdot)\right)\right\}_{i \in \mathbb{I}^{j}}\right)\right\}_{j \in \mathbb{J}},
$$

where $\mathbb{J}$ is an index set for the MB components, $w^{j}$ denotes the weight (probability) of the $j$ th MB component, $\mathbb{I}^{j}$ is an index set for the Bernoullis in the $j$ th $\mathrm{MB}$, and $r^{j, i}, f^{j, i}(\cdot)$ denote the parameters of the $i$ th Bernoulli in the $j$ th MB.

In MOT, the MBM is typically used to handle different data associations. Each MB density $f^{j}(\cdot)$ corresponds to a specific sequence of data associations, and the weight $w^{j}$ is the posterior probability of the data association sequence.

\subsubsection{Labeled Bernoulli}

A labeled object state $\tilde{\mathbf{x}}=(\mathbf{x}, \ell)$ can be understood as an object state $\mathbf{x}$ to which one has attached a label $\ell \in \mathbb{L}$, where $\mathbb{L}$ is the label space. A labeled Bernoulli RFS $\tilde{\mathbf{X}}$ is a special case of a Bernoulli RFS that, in addition to the probability of existence $r$ and the object state pdf $p(\cdot)$ also has a label $\ell \in \mathbb{L}$. The labeled Bernoulli density can be defined as

$$
f(\tilde{\mathbf{X}})=\left\{\begin{array}{ll}
1-r & \tilde{\mathbf{X}}=\emptyset \\
r f(\mathbf{x}) \Delta_{\ell}(l) & \tilde{\mathbf{X}}=\{(\mathbf{x}, l)\} \\
0 & |\tilde{\mathbf{X}}| \geq 2
\end{array} .\right.
$$

The labeled Bernoulli density is defined entirely by the three labeled Bernoulli parameters

$$
(\ell, r, f(\cdot)) \text {. }
$$

Analogously, labeled MB and labeled MBM are special cases of MB RFS and MBM RFS, where the Bernoulli labels are unique. In MOT, labeled Bernoulli can be used to form trajectories, i.e., sequences of states, by linking object estimates with the same label.

\subsection{STANDARD MODELS FOR MULTIPLE EXTENDED OBJECT TRACKING}

In this section we review the standard models for multiple extended object tracking. The dynamic model describes how the set of objects changes over time, i.e., describes how objects move and how they appear and disappear in the surveillance area. The measurement model describes both the measurements from the objects, as well as the false measurements, also called clutter.

\subsubsection{Standard dynamic model}

The dynamic model describes how the set of objects changes over time, from time step $k$ to time step $k+1$. There are three types of changes: new objects appear in the surveillance area, 
some existing objects disappear from the surveillance area, and some existing objects remain in the surveillance area.

New objects that appear in the surveillance area are either the result of object birth, or the result of object spawning. Object birth is a new object that appears, and the standard model for object birth is to model the set of born objects as a PPP with birth intensity

$$
\lambda_{k+1}^{\mathrm{b}}\left(\mathbf{x}_{k+1}\right)
$$

Object spawning is when a new object spawns from an existing one; object spawning has been rare in extended object tracking, however, spawning modeling for the random matrix model was presented in [112].

The probability of survival

$$
P^{\mathrm{S}}\left(\mathbf{x}_{k}\right)
$$

models the probability that an object with state $\mathbf{x}_{k}$ at time step $k$ survives to time step $k+1$, i.e., remains in the surveillance area. Conversely, $1-P^{\mathrm{S}}\left(\mathbf{x}_{k}\right)$ is the probability that the object dies, i.e., leaves the surveillance area. If the object remains in the surveillance area, then its state evolves according to a Markov process with transition density (7).

Note that this transition density describes both how the kinematic properties, such as position, velocity, acceleration and turn-rate, and the extent properties evolve over time. The standard assumption is that the objects that remain in the surveillance area evolve independently of each other, and independent of any objects that are born.

In summary, the standard dynamic model consists of three basic building blocks: the PPP birth intensity $\lambda_{k+1}^{\mathrm{b}}\left(\mathbf{x}_{k+1}\right)$, the probability of survival $P^{\mathrm{S}}\left(\mathbf{x}_{k}\right)$, and the transition density $f_{k+1, k}\left(\mathbf{x}_{k+1} \mid \mathbf{x}_{k}\right)$.

\subsubsection{Standard extended object measurement model}

The set of measurements consists of false alarm measurements and the measurements generated by the objects. Conditioned on the set of objects $\mathbf{X}$, the set of measurements can be expressed as

$$
\mathbf{Z}=\mathbf{K} \cup\left(\cup_{\mathbf{x} \in \mathbf{X}} \mathbf{W}(\mathbf{x})\right)
$$

where $\mathbf{K}$ is the (possibly empty) set of clutter measurements, and $\mathbf{W}(\mathbf{x})$ is the (possibly empty) set of measurements generated by the object with state $\mathbf{x}$. It is standard in MOT to assume that the sets are independent, i.e., the clutter set $\mathbf{K}$ is independent of the object measurements, and the object measurements are independent of each other. Furthermore, it is standard to assume that the measurement origin is unknown, i.e., that the data association is unknown.

The standard model for the clutter measurements $\mathbf{K}$ is a PPP with intensity $\kappa(\mathbf{z})$, giving the clutter density

$$
f(\mathbf{K})=e^{-\langle\kappa ; 1\rangle} \prod_{\mathbf{z} \in \mathbf{K}} \kappa(\mathbf{z}) .
$$

A model for the RFS measurement set $\mathbf{W}(\mathbf{x})$ (given the object state $\mathbf{x}$ ) incorporates both the spatial distribution of each individual measurement and the total number of measurements. Typical models assume that the measurements in $\mathbf{W}(\mathbf{x})$ are statistically independent given the object state $\mathbf{x}$. A widely-used model is the zero-inflated PPP model, see [17], [113]. It assumes that an object is detected with probability of detection

$$
P^{\mathrm{D}}(\mathbf{x})
$$

In case the object is detected, the received measurement set is distributed according to a PPP with intensity function

$$
\gamma(\mathbf{x}) \cdot \phi(\cdot \mid \mathbf{x})
$$

where $\gamma(\mathbf{x})$ is the Poisson rate that describes how many measurements are expected from an object with state $\mathbf{x}$, and

$$
\phi(\cdot \mid \mathbf{x})
$$

is a conditional spatial distribution as introduced in Eq. 9 that describes how, given an object state $\mathbf{x}$, the measurements are distributed around the object extent.

The zero-inflated PPP model can be applied in many different scenarios. For an object with state $\mathbf{x}$, the measurement likelihood is defined as

$$
f(\mathbf{W} \mid \mathbf{x})=\left\{\begin{array}{ll}
\ell_{\emptyset}(\mathbf{x}) & \mathbf{W}=\emptyset \\
\ell_{\mathbf{W}}(\mathbf{x}) & \text { else }
\end{array},\right.
$$

where

$$
\begin{aligned}
\ell_{\emptyset}(\mathbf{x}) & =1-P^{\mathrm{D}}(\mathbf{x})+P^{\mathrm{D}}(\mathbf{x}) e^{-\gamma(\mathbf{x})} \\
\ell_{\mathbf{W}}(\mathbf{x}) & =P^{\mathrm{D}}(\mathbf{x}) e^{-\gamma(\mathbf{x})} \prod_{\mathbf{z} \in \mathbf{W}} \gamma(\mathbf{x}) \phi(\mathbf{z} \mid \mathbf{x}) .
\end{aligned}
$$

In summary, the standard extended object measurement model consists of three basic building blocks: the PPP clutter intensity $\kappa(\mathbf{z})$, the probability of detection $P^{\mathrm{D}}(\mathbf{x})$, and the PPP object measurement intensity $\gamma(\mathbf{x}) \phi(\mathbf{z} \mid \mathbf{x})$.

Note that an unknown Poisson rate [114] can be modeled with the Gamma distribution, in order to estimate it.

\subsection{PROBLEM DEFINITION: MULTIPLE EXTENDED OBJECT TRACKING}

Next, we provide a definition of the MEOT problem. Let the set of objects at time step $k$ be denoted $\mathbf{X}_{k}$, let the set of measurements at time step $k$ be denoted $\mathbf{Z}_{k}$, and let $\mathbf{Z}_{1: k}$ denote the sequence of sets of measurements, from time step 1 to time step $k$. The multi-object posterior density for the set of objects conditioned on the measurement sets is denoted

$$
f_{k \mid k^{\prime}}\left(\mathbf{X}_{k} \mid \mathbf{Z}_{1: k^{\prime}}\right)
$$

where $k^{\prime}<k$ gives a predicted density, and $k^{\prime}=k$ gives a filtered density.

The multi-object Bayes filter propagates the multi-object density in time using the prediction

$$
\begin{aligned}
& f_{k \mid k-1}\left(\mathbf{X}_{k} \mid \mathbf{Z}_{1: k-1}\right)= \\
& \int f_{k, k-1}\left(\mathbf{X}_{k} \mid \mathbf{X}_{k-1}\right) \cdot f_{k-1 \mid k-1}\left(\mathbf{X}_{k-1} \mid \mathbf{Z}_{1: k-1}\right) \delta \mathbf{X}_{k-1}
\end{aligned}
$$




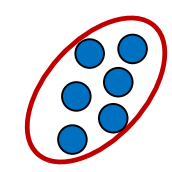

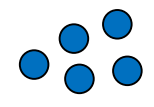

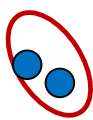

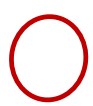

O

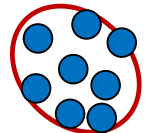

FIGURE 12. Illustration of the data association problem. There are four predicted objects (red ellipses) and 23 measurements (blue circles). Due to the unknown data association, it is not known which measurements belong together, and which measurements are from the same object. For this simple example, it is fairly easy to reason about which measurements likely belong together, and which measurements are from which predicted object. However, many real scenarios are much more complicated. The total number of theoretically possible data associations for this example is $258^{\prime} 153$, see Section 8.3 For methods to handle the data association in a computationally tractable way, see Section 9

The predicted RFS is updated with the measurement set $\mathbf{Z}_{1: k}$ according to the Bayes rule for random finite sets

$$
\begin{aligned}
& f_{k \mid k}\left(\mathbf{X}_{k} \mid \mathbf{Z}_{1: k}\right)= \\
& \frac{f_{k}\left(\mathbf{Z}_{k} \mid \mathbf{X}_{k}\right) \cdot f_{k \mid k-1}\left(\mathbf{X}_{k} \mid \mathbf{Z}_{1: k-1}\right)}{\int f_{k}\left(\mathbf{Z}_{k} \mid \mathbf{X}_{k}\right) \cdot f_{k \mid k-1}\left(\mathbf{X}_{k} \mid \mathbf{Z}_{1: k-1}\right) \delta \mathbf{X}_{k}},
\end{aligned}
$$

where $f_{k+1, k}\left(\mathbf{X}_{k+1} \mid \mathbf{X}_{k}\right)$ is the multi-object transition density, based on the standard dynamic model, Section 7.2.1 and $f_{k}\left(\mathbf{Z}_{k} \mid \mathbf{X}_{k}\right)$ is the measurement set density, based on the standard measurement model, Section 7.2.2 The integrals in (94) are set-integrals, which are defined in [9. Sec. 11.3.3].

The objective of an MOT algorithm is to recursively compute the multi-object density using the Bayes recursion 94. For this purpose, the predicted multi-object density (94a) and updated multi-object density (94b) should be in the same family of multi-object densities. This property is also called conjugacy and in this case, the predicted density is called conjugate prior. Two extended object multi-object conjugate priors are known, the Poisson Multi-Bernoulli Mixture (PMBM) density [103], and the delta-Generalized Labeled Multi-Bernoulli ( $\delta$-GLMB) density [115], [116].

Due to the unknown data association, the exact recursion is computationally intractable, and any MOT algorithm requires approximations. In the next section, we present the data association problem for multiple extended objects, and in Section 9 we present different methods for handling the data association. In Section 10 we review different computationally tractable MOT algorithms.

\section{ANALYSIS OF EXTENDED OBJECT DATA ASSOCIATION}

In this section we will first describe what the data association problem is, and then we will present a definition of multiple extended object data association. Next, we provide theoretical expressions for the number of possible data associations. It will be shown that this number is prohibitively large, and for computational tractability approximations are necessary. Lastly, we provide an analysis of the approximation error incurred when the posterior density is approximated.
Different approaches to handling the data association problem in extended object мот are presented in Section 9.

\subsection{THE DATA ASSOCIATION PROBLEM}

According to the Bayes update $94 \mathrm{~b}$, the posterior multiobject density is a mixture multi-object density. Each component in the mixture represents a sequence of associations, where the sequence contains the associations from the first to the current time step. The posterior density for the set of objects $\mathbf{X}$ can be described compactly as

$$
f(\mathbf{X} \mid \mathbf{Z})=\sum_{A \in \mathcal{A}} \mathcal{W}_{A} f(\mathbf{X} \mid A)
$$

where

$$
\begin{aligned}
\mathcal{W}_{A} & =\frac{\mathcal{L}_{A}}{\sum_{A^{\prime} \in \mathcal{A}} \mathcal{L}_{A^{\prime}}}, \text { and } \\
\mathcal{L}_{A} & =\int f(\mathbf{Z}, A \mid \mathbf{X}) f(\mathbf{X}) \delta \mathbf{X} \\
& =\int f(\mathbf{Z} \mid A, \mathbf{X}) f(A \mid \mathbf{X}) f(\mathbf{X}) \delta \mathbf{X}
\end{aligned}
$$

with prior density $f(\mathbf{X})$, finite association space $\mathcal{A}$, predicted likelihood $\mathcal{L}_{A}$ with $A \in \mathcal{A}$, and association probability $\mathcal{W}_{A}$ with $\sum_{A \in \mathcal{A}} \mathcal{W}_{A}=1$.

As the number of feasible associations increases (even more than) exponentially with the number of measurements and objects, see (107), there are two major challenges:

1) The number of mixture components in (95a) is large. In a tracking algorithm, it is not possible to exhaustively enumerate all data associations.

2) The hypothesis weights 95b are generally difficult to compute. The reason is that the normalization requires a sum over all data associations $A \in \mathcal{A}$. On the contrary, the association likelihoods $\mathcal{L}_{A}$ 95c can typically be computed straightforwardly, either exactly or using approximations.

The data association problem is illustrated in Figure 12

The standard approach to these challenges, both in extended object tracking and in point object tracking, is to approximate the exact mixture density with a mixture density with a low and constant number of components. The mixture density approximation

$$
f_{\hat{\mathcal{A}}}(\mathbf{X})=\sum_{A \in \hat{\mathcal{A}}} \hat{\mathcal{W}}_{A} f(\mathbf{X} \mid A)
$$

incorporates a subset of the associations $\hat{\mathcal{A}} \subseteq \mathcal{A}$, and the corresponding approximate association weights $\hat{\mathcal{W}}_{A}$, where the weights are normalized according to $\sum_{A \in \hat{\mathcal{A}}} \hat{\mathcal{W}}_{A}=1$ and $\hat{\mathcal{W}}_{A^{\prime}}=0$ for all $A^{\prime} \notin \hat{\mathcal{A}}$.

Arguably, the most common alternative, see, e.g., [103], [111], [116]-[118] for computing the approximate association weights $\mathcal{W}_{A}$ is

$$
\hat{\mathcal{W}}_{A}^{\mathrm{L}}=\frac{\mathcal{L}_{A}}{\sum_{A^{\prime} \in \hat{\mathcal{A}}} \mathcal{L}_{A^{\prime}}}
$$

for $A \in \mathcal{A}$. Alternative weight approximations are discussed in [119]. 


\subsection{DEFINITION OF DATA ASSOCIATION}

In MEOT an object may be associated to multiple measurements. From this it follows that the data association can be interpreted as having two parts:

1) Partition of the set of measurements: All measurements that have the same origin (i.e., object or clutter) can be grouped together. By this means, a partitioning of the measurement set is performed, i.e., a grouping of the measurements into non-empty and mutually disjoint subsets (named cells), such that each measurement belongs to exactly one cell.

2) Assignment of cells to objects: Each measurement cell can be assigned to a possible source (objects or clutter). This assignment is similar to data association for point object MOT: at most one cell can be associated to an object.

Given a partition of the measurements, and an assignment of the cells to sources, we have a complete extended object data association. In the following, the set of objects $\mathbf{X}$ is indexed by $i \in \mathbb{I}$, i.e.,

$$
\mathbf{X}=\left\{\mathbf{x}^{i}\right\}_{i \in \mathbb{I}}
$$

and the measurements in the set $\mathbf{Z}$ are indexed by $m \in \mathbb{M}$, i.e.,

$$
\mathbf{Z}=\left\{\mathbf{z}^{m}\right\}_{m \in \mathbb{M}}
$$

where $\mathbb{M} \cap \mathbb{I}=\emptyset$.

A data association $A \in \mathcal{A}$ assigns each measurement index in $\mathbb{M}$ to a source (either an existing object with index in $\mathbb{I}$ or a potential new object). Mathematically, a data association $A \in \mathcal{A}$ partitions $\mathbb{M} \cup \mathbb{I}$ into cells $C \in A$.

All measurements in a cell are assigned to the same source and a measurement comes from at most one target object. For this reason, an index cell contains either no or a single object index, i.e., $C \cap \mathbb{I}^{j} \neq \emptyset \Rightarrow\left|C \cap \mathbb{I}^{j}\right|=1$ for all $C \in A$.

Example 8.1: We consider three measurements and two objects, i.e., the index sets are $\mathbb{M}=\left\{m_{1}, m_{2}, m_{3}\right\}$ and $\mathbb{I}=\left\{i_{1}, i_{2}\right\}$. An example association, i.e., partition of $\mathbb{M} \cup \mathbb{I}$, is

$$
A=\left\{\left\{i_{1}, m_{1}, m_{2}\right\},\left\{m_{3}\right\},\left\{i_{2}\right\}\right\}
$$

where $\left\{i_{1}, m_{1}, m_{2}\right\},\left\{m_{3}\right\}$ and $\left\{i_{2}\right\}$ are the cells. In this example association, the measurements $m_{1}, m_{2}$ are associated to object $i_{1}$, measurement $m_{3}$ is clutter, and object $i_{2}$ is undetected. Note that measurement $m_{3}$ is a potential new object (i.e., clutter or an object that was not yet detected).

In the next example we provide all possible data associations for a small scenario with two measurements and one object.
TABLE 5. $\left\{\begin{array}{l}M \\ N\end{array}\right\}$ - Stirling number of the second kind

\begin{tabular}{c|rrrrrrrrr}
$M \backslash N$ & 0 & 1 & 2 & 3 & 4 & 5 & 6 & 7 & 8 \\
\hline 0 & 1 & & & & & & & & \\
1 & 0 & 1 & & & & & & & \\
2 & 0 & 1 & 1 & & & & & & \\
3 & 0 & 1 & 3 & 1 & & & & & \\
4 & 0 & 1 & 7 & 6 & 1 & & & & \\
5 & 0 & 1 & 15 & 25 & 10 & 1 & & & \\
6 & 0 & 1 & 31 & 90 & 65 & 15 & 1 & & \\
7 & 0 & 1 & 63 & 301 & 350 & 140 & 21 & 1 & \\
8 & 0 & 1 & 127 & 966 & 1701 & 1050 & 266 & 28 & 1
\end{tabular}

TABLE 6. $B(M)$ - The $M$ th Bell number

\begin{tabular}{c|rrrrrrrrr}
$M$ & 0 & 1 & 2 & 3 & 4 & 5 & 6 & 7 & 8 \\
\hline$B(M)$ & 1 & 1 & 2 & 5 & 15 & 52 & 203 & 877 & 4140
\end{tabular}

Example 8.2: We consider two measurements and one object, i.e., the index sets are $\mathbb{M}=\left\{m_{1}, m_{2}\right\}$ and $\mathbb{I}=\left\{i_{1}\right\}$. Then, there are five possible associations with the following cells:

$$
\begin{array}{ll}
A^{1}=\left\{\left\{m_{1}, m_{2}, i_{1}\right\}\right\} & \\
A^{2}=\left\{\left\{m_{1}, m_{2}\right\},\right. & \left.\left\{i_{1}\right\}\right\} \\
A^{3}=\left\{\left\{m_{1}, i_{1}\right\},\right. & \left.\left\{m_{2}\right\}\right\} \\
A^{4}=\left\{\left\{m_{1}\right\},\right. & \left.\left\{m_{2}, i_{1}\right\}\right\} \\
A^{5}=\left\{\left\{m_{1}\right\},\right. & \left.\left\{m_{2}\right\}, \quad\left\{i_{1}\right\}\right\}
\end{array}
$$

We see that the two measurements can either be in the same cell $\left(A^{1}\right.$ and $\left.A^{2}\right)$, or in different cells $\left(A^{3}, A^{4}\right.$ and $\left.A^{5}\right)$, and the object can either be associated to both measurements $\left(A^{1}\right)$, associated to one of the measurements $\left(A^{3}\right.$ and $\left.A^{4}\right)$, or associated to no measurement $\left(A^{2}\right.$ and $\left.A^{5}\right)$.

\subsection{COMPLEXITY OF DATA ASSOCIATION}

In Example 8.2 there are two measurements and one object, and this gives five possible data associations. Based on this example, we provide general expressions for the complexity of the data association.

\subsubsection{Number of partitions of a set}

A partition of a set $\mathbb{M}$ with $M$ elements can consist of 1 to $M$ cells. In the first extreme case of 1 cell, all measurements belong to the same cell. In the second extreme case of $M$ cells, a cell consists of exactly one measurement.

The number of different possibilities to partition a set $\mathbb{M}$ with $M$ elements into $N$ cells results from the Stirling number of the second kind [120, p. 244]

$$
\left\{\begin{array}{c}
M \\
N
\end{array}\right\}=\frac{1}{N !} \sum_{j=0}^{N}(-1)^{N-j}\left(\begin{array}{c}
N \\
j
\end{array}\right) j^{M} .
$$

Table 5 depicts the first Stirling numbers of the second kind. 
TABLE 7. Number of assignments of $N$ cells and $I$ objects

\begin{tabular}{c|rrrrrr}
$N \backslash I$ & 0 & 1 & 2 & 3 & 4 & 5 \\
\hline 0 & 1 & 1 & 1 & 1 & 1 & 1 \\
1 & 1 & 2 & 3 & 4 & 5 & 6 \\
2 & 1 & 3 & 7 & 13 & 21 & 31 \\
3 & 1 & 4 & 13 & 34 & 73 & 136 \\
4 & 1 & 5 & 21 & 73 & 209 & 501 \\
5 & 1 & 6 & 31 & 136 & 501 & 1546
\end{tabular}

Furthermore, the total number of partitions for a set with $M$ elements results from the $M$ th Bell number [121]

$$
B(M)=\sum_{N=1}^{M}\left\{\begin{array}{l}
M \\
N
\end{array}\right\} .
$$

As shown by the first Bell numbers depicted in Table 6 $B(M)$ increases rapidly with a growing $M$. Already for $M=90$ measurements, there are more than $10^{100}$ valid partitions.

\subsubsection{Number of cell-to-object assignments}

In the following, we assume there are $I=|\mathbb{I}|$ objects and $N$ cells in the measurement partition. The number of cells that can be associated to objects is between 0 and $\min (N, I)$, where the remaining cells are clutter. In the same way, the number objects that can be associated to a cell is also between 0 and $\min (N, I)$, where the remaining objects are misdetections. The number of possibilities to choose $T \leq \min (N, I)$ cells from $N$ cells results from the Binomial coefficient

$$
\left(\begin{array}{l}
N \\
T
\end{array}\right)=\frac{N !}{T !(N-T) !} .
$$

The number of possibilities to choose $T \leq \min (N, I)$ objects from $I$ objects results from the Binomial coefficient

$$
\left(\begin{array}{l}
I \\
T
\end{array}\right)=\frac{I !}{T !(I-T) !} .
$$

Finally, there are $T$ ! possibilities to associate the $T$ cells to the $T$ objects, and the number of cell-to-object assignments is given by

$$
\sum_{T=0}^{\min (N, I)}\left(\begin{array}{l}
N \\
T
\end{array}\right)\left(\begin{array}{l}
I \\
T
\end{array}\right) T !
$$

Starting from $N=0$ and $I=0$, the first number of assignments are depicted in Table 7

\subsubsection{Number of data associations}

Consider $I=|\mathbb{I}|$ previously detected objects, and $M=|\mathbb{M}|$ detections. The number of possibilities to associate $M$ detections to $I$ objects (or to clutter) is given by the number of partitions. The total number of data associations results from the number of partitions, and the number of cell-to-object assignments according to

$$
N_{\mathcal{A}}(M, I)=\sum_{N=1}^{M}\left\{\begin{array}{l}
M \\
N
\end{array}\right\} \sum_{T=0}^{\min (N, I)}\left(\begin{array}{l}
N \\
T
\end{array}\right)\left(\begin{array}{l}
I \\
T
\end{array}\right) T ! .
$$

The first number of data associations are given in Table 8 .
TABLE 8. $N_{\mathcal{A}}(M, I)$ - Number of extended object data associations for $M$ measurements and $I$ objects

\begin{tabular}{c|rrrrrr}
$M \backslash I$ & 0 & 1 & 2 & 3 & 4 & 5 \\
\hline 0 & 1 & 1 & 1 & 1 & 1 & 1 \\
1 & 1 & 2 & 3 & 4 & 5 & 6 \\
2 & 2 & 5 & 10 & 17 & 26 & 37 \\
3 & 5 & 15 & 37 & 77 & 141 & 235 \\
4 & 15 & 52 & 151 & 372 & 799 & 1540 \\
5 & 52 & 203 & 674 & 1915 & 4736 & 10427
\end{tabular}

\subsection{APPROXIMATION ERROR UPPER BOUND}

Approximating the exact density (95a) with the approximation (96) will inevitably incur an approximation error. There are different alternatives for measuring the size of the approximation error, one alternative is the $L_{1}$-norm

$$
\left\|f-f_{\hat{\mathcal{A}}}\right\|_{1} \text {. }
$$

By using the triangle inequality it can be shown that the $L_{1}$ error of the density approximation has an upper bound [119]

$$
\left\|f-f_{\hat{\mathcal{A}}}\right\|_{1} \leq \sum_{A \in \mathcal{A} \backslash \hat{\mathcal{A}}} \mathcal{W}_{A}+\sum_{A \in \hat{\mathcal{A}}} \operatorname{abs}\left(\mathcal{W}_{A}-\hat{\mathcal{W}}_{A}\right)
$$

In (109) equality holds, e.g., if there is no overlap in the support between the conditional densities $f(\cdot \mid A)$ and $f_{\hat{\mathcal{A}}}\left(\cdot \mid A^{\prime}\right)$, i.e., for all $A \in \mathcal{A}$ and $A^{\prime} \in \mathcal{A}$ with $A \neq A^{\prime}$ it holds that

$$
\operatorname{supp}(f(\cdot \mid A)) \cap \operatorname{supp}\left(f\left(\cdot \mid A^{\prime}\right)\right)=\emptyset .
$$

The upper bound on the $L_{1}$-error (109) encodes the following information about the approximation error:

1) The truncated associations, i.e., which associations are not included in the subset $\hat{\mathcal{A}}$

$$
\sum_{A \in \mathcal{A} \backslash \hat{\mathcal{A}}} \mathcal{W}_{A}
$$

2) The approximate association weights $\hat{\mathcal{W}}_{A}$

$$
\sum_{A \in \hat{\mathcal{A}}} \operatorname{abs}\left(\mathcal{W}_{A}-\hat{\mathcal{W}}_{A}\right)
$$

For an approximation of the weights according to [97, the upper bound on the $L_{1}$-error is [119, Sec. VI.C]

$$
\left\|f-f_{\hat{\mathcal{A}}}\right\|_{1} \leq 2 \sum_{A \in \mathcal{A} \backslash \hat{\mathcal{A}}} \mathcal{W}_{A} .
$$

From this we can draw the conclusion that by pruning data associations that are improbable, i.e., have small weights, the approximation error becomes small. In [119, Sec. VI.C] it is shown that the approximation error upper bound (113) can be made arbitrarily small by including in $\hat{\mathcal{A}}$ the associations that have high likelihoods, and pruning the rest. In other words, the method used to compute the subset of associations $\hat{\mathcal{A}}$ should be designed to find the associations with large weights (95b), i.e., large likelihoods 95c. In the next section, we review different methods to compute a subset of data associations such that the subset indeed contains associations with large likelihoods. 


\section{EXTENDED OBJECT DATA ASSOCIATION METHODS}

In the following, we present different approaches to obtain the subset $\hat{\mathcal{A}}$ in Eq. 96). The complexity of the data association problem for extended objects is inherently combinatorial, and for computational tractability, it is necessary to use approximations in an MEOT algorithm. Similarly to point object tracking, the purpose of a data association method is to prune the improbable data associations, such that a computationally tractable number of highly probable data associations remain. Existing approaches can be grouped into three categories: gating, clustering methods combined with optimal assignment algorithms, and random sampling based methods that solve partitioning and assignment jointly.

\subsection{GATING}

The idea of gating [122, Sec. 2.2.2.2] is to prune highly improbable measurement-to-object associations. Gating is used commonly not only in extended object tracking, but also in point object tracking. Gating builds upon forming a probabilistic area around the predicted object state, and to only allow associations of measurements that are located in the area, i.e., that fall inside the gate. Arguably, the most common variant of gating is ellipsoidal gating for Gaussian object state densities. An important difference between point object gating and extended object gating is that for extended objects, one must consider not only the predicted object position, but also the predicted extent (shape and size), as well as the corresponding variances. An illustration of gating for a rectangular extended object is presented in Figure 13 , note how the gate takes the object shape into account.

In addition to pruning highly improbable associations, with gating it is also possible to partition the tracking problem into smaller sub-problems. It is often computationally cheaper to track several groups of few targets, than to track a single group of many targets, and the grouping also enables parallelization.

\subsection{CLUSTERING FOLLOWED BY ASSIGNMENT}

In many works [103], [111], [116]-[118], $\hat{\mathcal{A}}$ is obtained using a two-step approach, where, first clustering is used to find a subset of partitions, and second, for each partition, optimal assignment is employed to compute a data association.

\subsubsection{Clustering to find partitions}

The first approaches to finding data associations with higher probability were based on using clustering algorithms to find partitions of the measurements with higher probability. The basic intuition behind using clustering has to do with the typical measurement process, where the measurements that were caused by an object are spatially distributed around the object extent. It follows from this spatial distribution that measurements that are spatially close, as measured by, e.g., the Euclidean distance, are more probable to be from the same object, and measurements that are spatially well separated are improbable to be from the same object. In Fig- ure 14 we given an example clustering of the measurements in Figure 12

Using clustering based on spatial proximity, one can compute a set of partitions, and prune remaining partitions, see Figure 15. By using clustering to compute a subset of partitions, there should (hopefully) be at least one partition that is sufficiently close to the true partition, such that the subsequent tracking yields accurate results.

An important contribution of the early works about RFS based MOT [117], [118] is to show that good tracking results can be achieved using clustering to find only a subset of partitions. Although there is often a significant reduction in the number of partitions that are incorporated, simulations and experiments show that the overall performance usually does not suffer significantly when clustering methods are employed [123]-[125].

Various clustering methods have been proposed in the literature. Distance Partitioning [117] is based on hierarchical single linkage clustering, where a measurement belongs to a cluster if the distance to the nearest neighbor in the cluster is less than a threshold. By using multiple thresholds, multiple partitions can be computed [117], [126]. Further clustering methods that have been proposed are Expectation Maximization (EM) [118], density based spatial clustering (DBSCAN) [127], spectral clustering [128], and fuzzy adaptive resonance theory [129], [130].

Determining a suitable subset of partitions (i.e., the most likely ones) is especially essential when multiple extended objects are closely-spaced; failure to find the right partitions in such scenarios may lead to merging extended objects, see, e.g., [111], [116], [118]. When multiple extended objects approach each other and become very close it is possible to use prior information about the expected number of measurements from one object, or predicted state information, to improve the partitioning, see, e.g., [118].

\subsubsection{Optimal, and sub-optimal, assignment algorithms}

Given a partition of the measurements, computed, e.g., using clustering, it is possible to compute a set of data associations by using optimal assignment algorithms to compute cellto-object assignments. In Figure 14 we give an example clustering and assignment of the predicted objects and measurements from Figure 12 The single best assignment can be computed using, e.g., the Hungarian algorithm [97]-[99] or the auction algorithm [131]. A ranked list of the $M$ best assignments can be computed using, e.g., Murty's algorithm [132]. A review of optimal assignment algorithms for MOT is provided in [133].

Murty's algorithm for finding the $M$ best assignments can be computationally costly, and a cheaper but sub-optimal alternative is to use so called Gibbs sampling [134]. Gibbs sampling is not guaranteed to return the $M$ best assignments, however, if a sufficient number of iterations are used in the Gibbs sampling, then it is possible to find $M$ good assignments such that good tracking performance can be achieved, see, e.g., [135], [136]. 

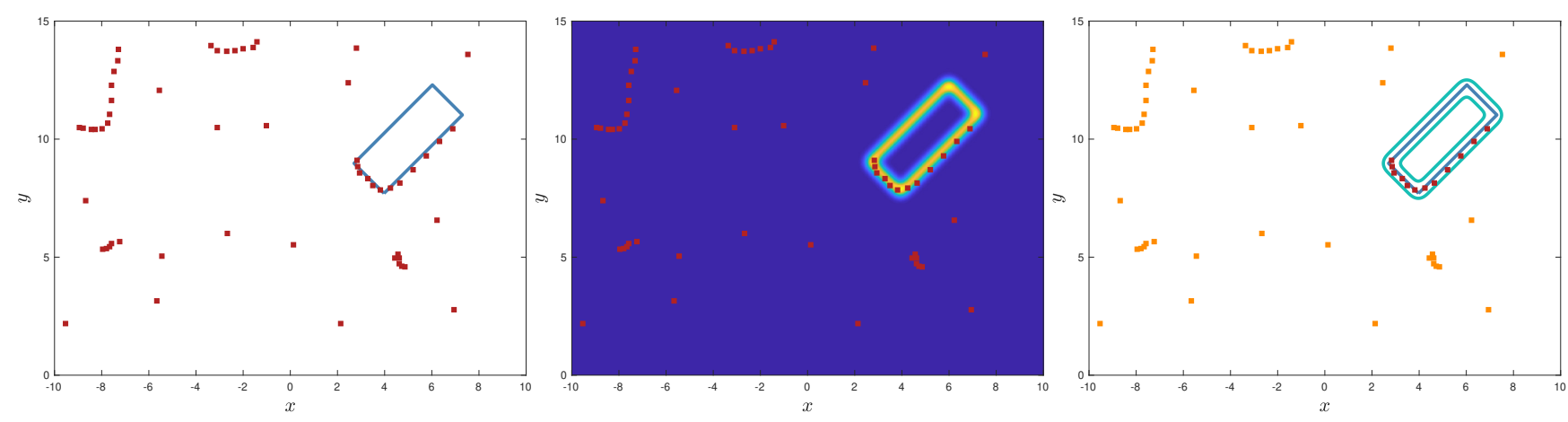

FIGURE 13. Example illustration of gating. Left: an object prediction (blue rectangle) and 57 measurements (red squares). Middle: Visualization of measurement likelihood, the warmer the color, the higher the likelihood. Note that self-occlusion was not taken into account in this example. Right: object prediction (blue rectangle), data association gates (cyan), measurements inside gate (red squares), and measurements outside gates (orange squares). Using gating, in this example 45 of the measurements can be rejected for this object prediction.

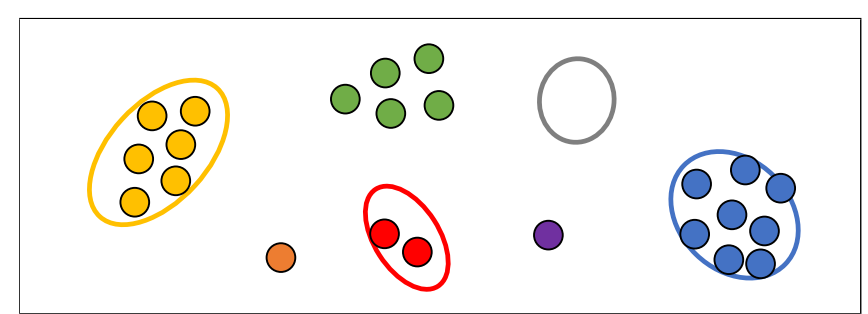

FIGURE 14. Example partition computed for the measurements in Figure 12 Measurements with the same color belong to the same cluster, i.e., the same cell in the partition. The color of the ellipse corresponds to the association; gray colored ellipses are not associated to any measurements, i.e., are misdetected.
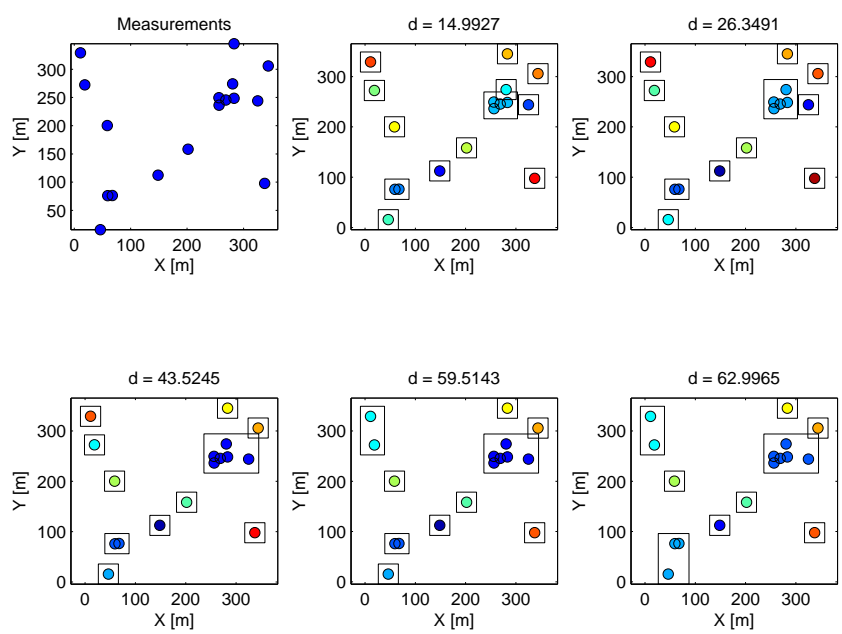

FIGURE 15. Example illustration of hierarchical clustering. Top left shows a scenario with 17 simulated measurements. Using Distance Partitioning [117] with different spatial proximity thresholds $d$, different clustering results are obtained. Note that the clustering results corresponds to a subset of the possible partitions of the measurements, and the clustering can thus be used to approximate the set of all partitions. A too small proximity threshold $d$ could lead to over-segmentation (too many clusters) of the measurements; analogously a too large $d$ could lead to under-segmentation (too few clusters) of the measurements. It is essential that the range of values of $d$ that are used will probably yield a subset of partitions, where at least one partition has a high overlap with the true partition. (Source: 117])

\subsection{SAMPLING}

It has been shown that a combination of clustering and assignment (C\&A) can yield convincing results at a tractable computational cost, see, e.g., [103], [111], [116]-[118]. However, some drawbacks of using C\&A were identified in [119]:

- The application of clustering algorithms builds upon intuition and heuristics, rather than building upon maximization of the predicted likelihood $95 \mathrm{c}$. In order to obtain as good approximations as possible, we wish to find data associations with high predicted likelihood, see Section 8, and it is not certain that the clustering will discover the highly probable data associations; instead empirical evaluation is required to verify this.

- When clustering is used, especially clustering methods that are based on spatial proximity, or density, it can be difficult to handle extended objects that are close to one another, see, e.g., [118]. In Fig. [16 we illustrate this. To handle this type of scenario, multiple clustering algorithms can be used, see, e.g., [118]. An example of this is shown in Fig. 17. This can alleviate the problems that arise when the objects are close, but also means that a complicated combination of clustering methods is necessary.

- The performance of the subsequent tracking is highly dependent on the clustering performance. Parameters tuned for one sensor or scenario may not be applicable to another sensor or scenario. A worst case scenario is that poorly chosen parameters lead to a degradation of the tracking results, with missed objects and/or false objects.

In [119], [137] it was shown that it is possible to solve the data association in a single step, instead of a two step approach with clustering followed by assignment. This is achieved using random sampling, where the main idea is to start from an initial data association, and then randomly modify the association to get a new data association. The probability of selecting a specific modification (called action) is based on the resulting predicted likelihood (95c) of the 


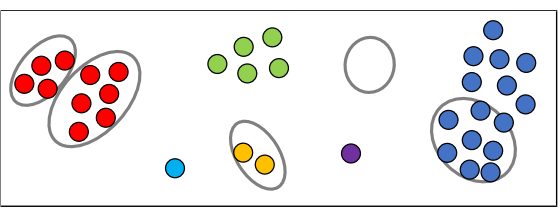

(a) Spatial proximity clustering

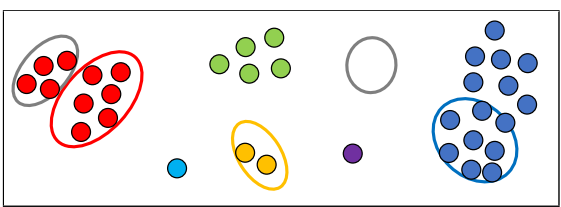

(b) Example assignment

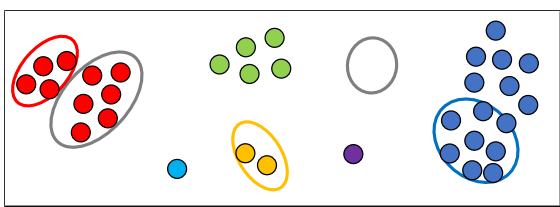

(c) Example assignment

FIGURE 16. Example illustration of when clustering and assignment may fail. In comparison to the simple scenario in Fig. 12 there are now additional measurements, and one additional predicted object. (a) When clustering based on spatial proximity is used, there is a risk that measurements from closely-spaced objects are merged. This has happened in the red cluster on the left, and the dark blue cluster on the right. (b) and (c) Assignments based on the clustering in (a). Note that in both cases, one of the two predicted objects on the left are incorrectly misdetected, on the right there is probably a new object that was misdetected, and the objects associated to the red and blue clusters will suffer from distortion due to being associated to too large clusters.

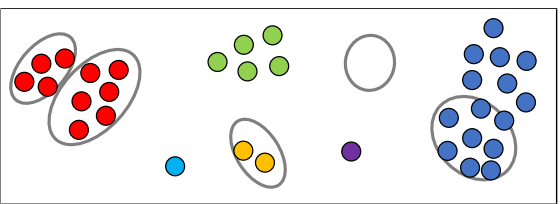

(a) Spatial proximity clustering

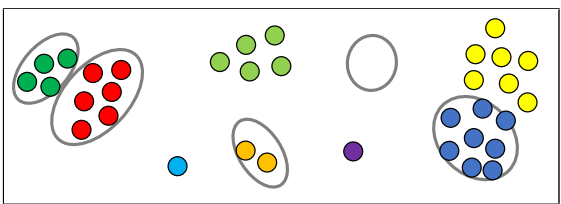

(b) Refined clustering based on predictions

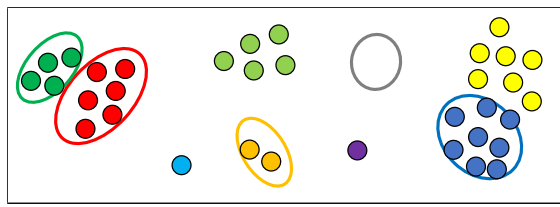

(c) Example assignment

FIGURE 17. Example illustration of how a hierarchy of clustering can improve performance, compared to Fig. 16 (a) Clustering based on spatial proximity. (b) Refined clustering based on the object predictions, where too large clusters have been split in accordance to the predictions. (c) Assignment based on the refined clustering.

new data association. The higher the likelihood $95 \mathrm{c}$ ) of the new data association, the higher the probability of randomly selecting the corresponding action. When an action has been selected, the procedure is repeated starting with the new association. From a sequence of such sampled data associations, a subset of associations with high predicted likelihoods (95c) can be obtained.

A few benefits of sampling methods were pointed out in [119], [137]:

- The partitioning and the cell-to-object assignment is handled jointly, and not in a two step fashion.

- The sampling works directly with the predicted likelihood (95c), which is related to the posterior data association weight (95b), which is what we wish to maximize.

- Given the measurements and a predicted likelihood, there is only a single parameter: the number of iterations.

Note that the sampling methods handle both the partitioning and the cell-to-object assignments jointly. Sampling methods that have been used for extended object data association include Gibbs sampling [137], Metropolis-Hastings (MH) [119], combination of Gibbs and MH [119], Stochastic optimization [119], and a combination of hierarchical clustering and Gibbs sampling [138].

\subsubsection{Markov Chain Monte Carlo}

The MCMC approach is based on the idea to sample from a target probability distribution by means of constructing a Markov Chain whose stationary distribution is equal to the target distribution. In the context of extended object tracking, it is useful to sample from the distribution over the data associations, $\mathrm{P}\{A\}$. For this purpose, two types of MCMC are especially relevant - Gibbs sampling and MH. Gibbs sampling is useful when it is difficult to sample directly from a distribution-in extended object data association, the prohibitively larger number of data associations make it practically impossible to sample from the distribution $\mathrm{P}\{A\}$. $\mathrm{MH}$ algorithms are useful when it is possible to sample from a distribution that can be evaluated up to a normalizing constant. Hence, MH is relevant for extended object tracking as we can evaluate the predicted likelihood (95c) but not the weight 95b).

When applied to extended object data association, Gibbs sampling changes the data association for a single measurement in each iteration. This allows for finer changes to the data association, but can be slow. With MH, clusters of measurements are either split or merged. This allows for larger changes and thus a faster algorithm, but finer changes are much more improbable. By combining the two-MH followed by refinement using Gibbs-it is possible to improve performance [119].

\subsubsection{Stochastic optimization}

Stochastic optimization [119] allows changes to a single measurement, as well as splits and merges, in each iteration. This leads to a sampling algorithm for extended object data association that, compared to Gibbs, $\mathrm{MH}$ and their sequential combination, is faster and more robust to the initial data association [119]. Example results from the stochastic optimization approach are shown in Figure 18 A downside to the mixing of Gibbs and $\mathrm{MH}$ in this way is that the sampling algorithm no longer satisfies all theoretical properties of an MCMC method, i.e., it cannot be guaranteed that in the limit, as the number of sampling iterations approach infinity, we are sampling from $\mathrm{P}\{A\}$. In [119] it is argued that this is not a major problem, because the primary purpose is not to accu- 
rately represent the distribution $\mathrm{P}\{A\}$, but to obtain a subset of data associations that have high predicted likelihoods.

\subsubsection{Hierarchical clustering and Gibbs sampling}

In [138] a combined clustering and sampling approach is presented. In automotive applications, using sensors like high resolution RADAR or LIDAR, it may be the case that data from objects closer to the sensor should be clustered in a different way than data from objects further from the sensor, see, e.g., [138, Fig. 1]. However, this can be difficult to achieve with a clustering method based on spatial proximity, which may yield multiple clustering results where some are accurate for objects closer to the sensor but inaccurate for objects farther from the sensor, or vice versa. To improve this, while still retaining the computational savings that clustering can give, in [138] it was proposed to first use a hierarchical clustering method to limit the degrees of freedom in the partitioning of the measurements, and then, given the hierarchical clustering, use Gibbs sampling to select partition cells from the hierarchy of partitions and assign them to the objects. A simulation study showed that hierarchical clustering and Gibbs sampling outperforms clustering followed by optimal assignment in two challenging scenarios [138].

The relationship to Gibbs, $\mathrm{MH}$ and SO can be explained as follows: by using hierarchical clustering first, the number of possible changes to the data association is greatly restricted. Changing just a single measurement (Gibbs step) is not possible, unless there is a cluster with a single measurement. The possible splits and merges (MH step) are restricted to only allow splits and merges that are already computed in the hierarchical clustering. Thanks to these restrictions, the computational cost of the data association is greatly reduced. However, because clustering is used, the data association is again subject to failures incurred during the clustering; note that clustering errors were one of the motivations for the sampling methods [119], [137]

\subsection{MARGINAL ASSOCIATION PROBABILITIES}

The multi-object posterior can be approximated using marginal association probabilities. For point object tracking, the Joint Probabilistic Data Association (JPDA) filter [139] is a popular tracking filter that builds upon marginal association probabilities. In this section we present a definition of marginal association probabilities, and review computationally tractable methods to compute them.

\subsubsection{Definition}

With a set of objects indexed by $i \in \mathbb{I}$ and a set of measurements indexed by $m \in \mathbb{M}$, there are at least two possible variants for the marginal association probabilities: the probability of associating a measurement to the object, or the probability of associating a cluster of measurements to the object.

First, the marginal association probability for object $i$ and measurement $m$ is denoted $\beta_{i, m}$ and is defined as

$$
\beta_{i, m}=\sum_{\substack{A \in \mathcal{A}: \\ \exists C \in A: i \in C, m \in C}} \mathcal{W}_{A} \propto \sum_{\substack{A \in \mathcal{A}: \\ \exists C \in A: i \in C, m \in C}} \mathcal{L}_{A},
$$

i.e., the sum of the data association probabilities $\mathcal{W}_{A}$ for all associations $A \in \mathcal{A}$ in which there is a cell $C \in A$ that includes both object $i$ and measurement $m$.

Second, the marginal association probability for object $i$ and a subset of measurements indexed by $m \in \tilde{\mathbb{M}} \subseteq \mathbb{M}$ is denoted $\beta_{i, \tilde{\mathbb{M}}}$ and is defined as

$$
\beta_{i, \tilde{\mathbb{M}}}=\sum_{\substack{A \in \mathcal{A}: \\ \exists C \in A: i \in C,(C \backslash i)=\tilde{\mathbb{M}}}} \mathcal{W}_{A} \propto \sum_{\substack{A \in \mathcal{A}: \\ \exists C \in A: i \in C,(C \backslash i)=\tilde{\mathbb{M}}}} \mathcal{L}_{A} .
$$

\subsubsection{Approximation}

Computing the marginal association probabilities, (114) or [115), requires a sum over all possible data associations, which has prohibitive complexity, and approximations are necessary. Similarly to how clustering and optimal assignment, Section 9.2 can be used to handle the data association, it is possible to use clustering as a pre-processing step, see [140], [141]. For a given set of clusters, it is then possible to use point object JPDA methods to compute the marginal association probabilities. An overview of approximate methods for computing point object marginal association probabilities is given in [142]. An approximation of the marginal association probabilities based on the sum-product-algorithm, without the need for pre-clustering, is presented in [143].

In [144] a slight modification of the standard dynamic and measurement models is considered, where the probability of detection is one $P^{\mathrm{D}}(\mathbf{x})=1 / 2$ the Poisson rate for each object is known $\gamma\left(\mathbf{x}^{i}\right)=\lambda^{i}$, and there is no object birth, i.e., $\lambda^{\mathrm{b}}(\mathbf{x})=0$. In this case, a data association $A$ divides the set of measurements into $|\mathbb{I}|+1$ subsets: one set $\mathbf{Z}_{c}$ associated to clutter and the remaining sets $\mathbf{Z}^{i}$ associated to the objects indexed by $i \in \mathbb{I}$. The predicted likelihood for the association $A$ can then be expressed as

$$
\mathcal{L}_{A} \propto \prod_{\mathbf{z} \in \mathbf{Z}_{c}} \kappa(\mathbf{z}) \prod_{i \in \mathbb{I}} \int\left[\prod_{\mathbf{z}^{i} \in \mathbf{Z}^{i}} \lambda^{i} \phi\left(\mathbf{z}^{i} \mid \mathbf{x}^{i}\right)\right] p\left(\mathbf{x}^{i}\right) \mathrm{d} \mathbf{x}^{i} .
$$

For each object we have the marginalization of the object state from the joint measurement-object density $p\left(\mathbf{Z}^{i} \mid \mathbf{x}^{i}\right) p\left(\mathbf{x}^{i}\right)$. By making a factorized approximation where each factor is the marginal density ${ }^{3}$ the predicted likelihood for the data association $A$ is approximated as [144, Eq. 11]

$$
\mathcal{L}_{A} \stackrel{\propto}{\sim} \prod_{\mathbf{z} \in \mathbf{Z}_{c}} \kappa(\mathbf{z}) \prod_{i \in \mathbb{I}} \prod_{\mathbf{z}^{i} \in \mathbf{Z}^{i}}\left[\int \lambda^{i} \phi\left(\mathbf{z}^{i} \mid \mathbf{x}^{i}\right) p\left(\mathbf{x}^{i}\right) \mathrm{d} \mathbf{x}^{i}\right] .
$$

\footnotetext{
${ }^{2}$ Note that there is still a non-zero probability of misdetection, as the PPP sample may be an empty measurement set.

${ }^{3}$ The optimal factorized density approximation, in the minimal KullbackLeibler sense, is the one where each factor is the marginal density.
} 


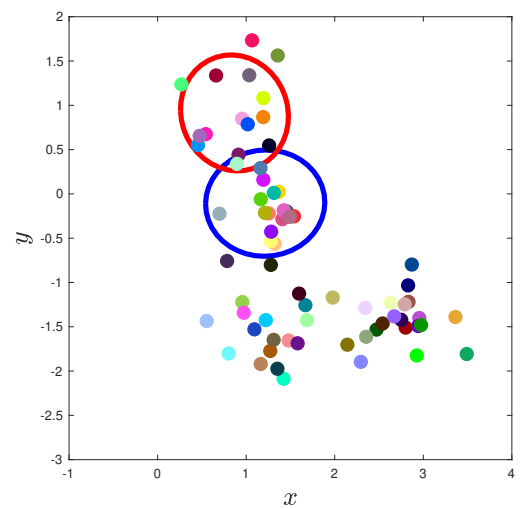

(a) Initial data association

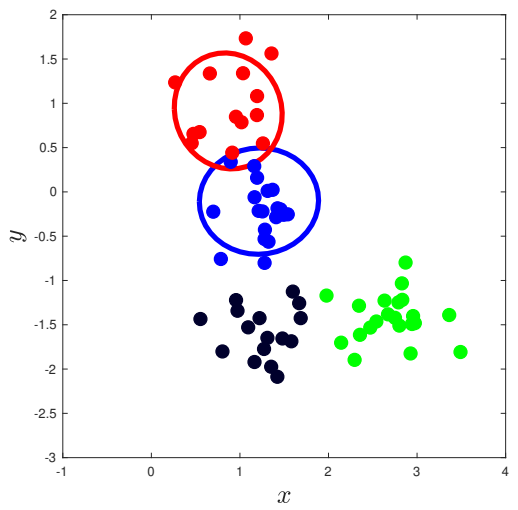

(b) Best data association

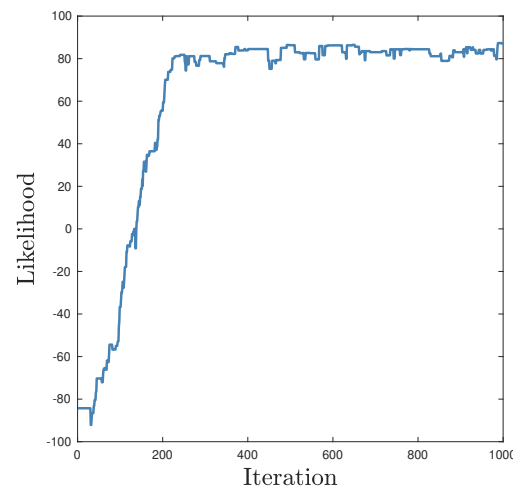

(c) Likelihood of data association

FIGURE 18. Example illustration of the stochastic optimization approach from [119]. (a) Two predicted objects with elliptic extent (red and blue ellipses) and 70 measurements colored according to the association. In this initial data association each measurement is associated to a potential new object, and both predicted objects are misdetected. Note that it is possible to initialize the sampling better than here, such that fewer iterations are necessary, see [119]. This initialization was chosen to illustrate that sampling based data association can converge even from quite poor initializations. (b) Best data association (highest likelihood) after running the stochastic optimization approach [119]. The colors correspond to the data association, i.e., both predicted objects have been associated to a cluster of measurements, and there are two potential new objects corresponding to the black and green clusters. (c) The likelihood of the data association; note the rapid increase during the first 200 iterations. (Source: [119])

Given the approximated predicted likelihood (117), the marginal association probability (114) can then be computed up to proportionality as [144] Eq. 15]

$$
\beta_{i, m} \propto \frac{\int \lambda^{i} \phi\left(\mathbf{z}^{m} \mid \mathbf{x}^{i}\right) p\left(\mathbf{x}^{i}\right) \mathrm{d} \mathbf{x}^{i}}{\kappa\left(\mathbf{z}^{m}\right)+\sum_{i^{\prime} \in \mathbb{I}} \int \lambda^{i^{\prime}} \phi\left(\mathbf{z}^{m} \mid \mathbf{x}^{i^{\prime}}\right) p\left(\mathbf{x}^{i^{\prime}}\right) \mathrm{d} \mathbf{x}^{i^{\prime}}} .
$$

An extension of this approximation for the case of missed detections was recently developed in [145]. Note that the assumption that the measurement rates $\bar{\lambda}^{i}$ are known can be relaxed by estimating the rates directly, see [114].

\section{ALGORITHMS FOR TRACKING MULTIPLE EXTENDED OBJECTS}

In general, there are two different basic concepts to approximate the intractable Bayesian filter equation (94) for the multi-object RFS density. First, instead of working directly with the multi-object RFS density, it is possible to recursively maintain a moment approximation of the RFS density as in the PHD filter and the CPHD filter. Second, a specific parameterized RFS density can be employed to approximate the exact RFS density as, for example, in the GLMB filter and the PMBM filter.

\subsection{PHD FILTER}

The PHD filter approximates the multi-object density under an assumption that the cardinality is Poisson distributed and that the objects are iid, where the normalized PHD is the object pdf; the PHD filter can thus be interpreted as a PPP approximation of the multi-object density 93.

A PHD filter for the standard measurement model was presented in [146]. The PHD filter presented in [147], [148] is derived for a measurement model where the objects are modeled by a Poisson cluster process with a Poisson parent process for the number of objects and a daughter process that models a number of measurement generating reflection points.

\subsubsection{Prediction and update}

In the extended object PHD filter [146], the Bayes recursion for the multi-object density 94 , is replaced by a predictionupdate recursion for the PHD, denoted $D_{k \mid k^{\prime}}\left(\mathbf{x}_{k}\right)$. Given a posterior PHD $D_{k-1 \mid k-1}\left(\mathbf{x}_{k-1}\right)$, for the standard dynamic model, Section 7.2.1, the predicted PHD is

$$
D_{k \mid k-1}\left(\mathbf{x}_{k}\right)=\lambda_{k}^{\mathrm{b}}\left(\mathbf{x}_{k}\right)+\left\langle f_{k, k-1} p_{\mathrm{S}} ; D_{k-1 \mid k-1}\right\rangle \text {. }
$$

In the update of the PHD filter we have the prior PHD $D_{k \mid k-1}\left(\mathbf{x}_{k}\right)$ and want to compute the posterior PHD. However, given a prior PPP, a set of measurements $\mathbf{Z}_{k}$, and the standard measurement model, the Bayes posterior density 94b is not a PPP, but a Poisson Multi-Bernoulli density [103. Thm. 2]. Therefore, approximations are necessary in the update step of the PHD filter. The approximated posterior PHD can be expressed as

$$
D_{k \mid k}\left(\mathbf{x}_{k}\right)=\ell_{\emptyset}\left(\mathbf{x}_{k}\right) D_{k \mid k-1}\left(\mathbf{x}_{k}\right)+\sum_{\mathbf{P} \in \mathcal{P}} \sum_{\mathbf{C} \in \mathbf{P}} \mathcal{W}_{\mathbf{P}} r_{k \mid k}^{\mathbf{C}} p_{k \mid k}^{\mathbf{C}}\left(\mathbf{x}_{k}\right)
$$

where $\mathcal{P}$ is the set of all possible partitions of the set of measurements $\mathbf{Z}_{k}, \mathbf{P} \in \mathcal{P}$ is a partition, $\mathbf{C} \in \mathbf{P}$ is a cell in the partition, and

$$
\begin{aligned}
r_{k \mid k}^{i, \mathbf{C}} & =\frac{\left\langle\ell_{\mathbf{C}} ; D_{k \mid k-1}\right\rangle}{\mathcal{L}_{\mathbf{C}}}, \\
p_{k \mid k}^{i, \mathbf{C}}\left(\mathbf{x}_{k}\right) & =\frac{\ell_{\mathbf{C}}\left(\mathbf{x}_{k}\right) D_{k \mid k-1}\left(\mathbf{x}_{k}\right)}{\left\langle\ell_{\mathbf{C}} ; D_{k \mid k-1}\right\rangle}, \\
\mathcal{L}_{\mathbf{C}} & =\Delta_{1}(|\mathbf{C}|) \kappa^{\mathbf{C}}+\left\langle\ell_{\mathbf{C}} ; D_{k \mid k-1}\right\rangle \\
\mathcal{W}_{\mathbf{P}} & =\frac{\prod_{\mathbf{C} \in \mathbf{P}} \mathcal{L}_{\mathbf{C}}}{\sum_{\mathbf{P}^{\prime} \in \mathcal{P}} \prod_{\mathbf{C}^{\prime} \in \mathbf{P}^{\prime}} \mathcal{L}_{\mathbf{C}^{\prime}}} .
\end{aligned}
$$


We see that the posterior intensity $(120)$ includes a summation over all possible partitions of the set of measurements $\mathbf{Z}_{k}$. In Section 8 we analyzed the complexity of this, and in Section 9 we presented methods to approximate the posterior intensity such that only a subset of partitions is necessary.

\subsubsection{Implementation}

The PHD filter is implemented using a density mixture representation of the PHD

$$
D_{k \mid k}(\mathbf{x})=\sum_{i \in \mathbb{I}_{k \mid k}} w_{k \mid k}^{i} p_{k \mid k}^{i}(\mathbf{x}) .
$$

It follows that the PHD filter recursion comes down to the prediction and update of the set of parameters

$$
\left\{\left(w_{k \mid k}^{i}, p_{k \mid k}^{i}(\cdot)\right)\right\}_{i \in \mathbb{I}_{k \mid k}} .
$$

The number of mixture components, i.e., the size of the index set $\mathbb{I}_{k \mid k}$, increases both in the prediction (119) due to the addition of the birth intensity (also represented by a mixture), and in the update (120) due to the sum over all possible partitions of the measurement set. For computational tractability, the implementations require mixture reduction, which is achieved by pruning of components with small weights, merging of similar components, and capping the total number of mixture components to some maximum number.

Gaussian mixture implementations of the extended object PHD filter, abbreviated ET-GM-PHD filters, are presented in [53], [117]. A Gaussian inverse Wishart implementation for the random matrix model [19], abbreviated GIW-PHD filter, is presented in [118], [149]. A Gaussian mixture implementation for the star-convex RHM based on Fourier coefficient is presented in [150], [151], and for the star-convex Cartesiandefined spline model it is proposed in [152]. Multiple model Gaussian mixture PHD filters are discussed in [123], [124], where it is demonstrated that multiple measurement models can lead to improved tracking results in extended object tracking. In [153], an extended target PHD filter based on sets of trajectories is presented.

If the Poisson rates $\gamma(\cdot)$ are unknown, then the implementation can be augmented with the Gamma distribution, which is the conjugate prior for the unknown Poisson rate, thus enabling the Poisson rate to be estimated, see [114]. The resulting implementations can be called Gamma Gaussian (GG), and Gamma Gaussian Inverse Wishart (GGIW), respectively. The Poisson distribution has a variance equal to the expected value, hence when the expected number of objects is high, the PHD filter also has high cardinality variance. It follows that the PHD is sensitive to both misdetections and to clutter, which often leads to missed objects or false objects, see, e.g., [117], [118]. By heuristically augmenting the PHD with labels it is possible to form labeled trajectories using post-processing, see, e.g., [125].

\subsection{CPHD FILTER}

In the Cardinalized Probability Hypothesis Density (CPHD) filter the multi-object density (93) is approximated by an iid cluster density. In comparison to the PHD filter, the objects are still assumed to be iid, however, the cardinality distribution is not restricted to any specific density.

The CPHD filter recursively maintains the PHD plus a truncated cardinality distribution. By this means, an improved cardinality estimate compared to the PHD filter is obtained.

The CPHD filter suffers from a "spooky effect" [116], which denotes a PHD mass shift from undetected objects to detected objects, even when the objects are far away from each other.

\subsubsection{Prediction and update}

In the CPHD filter the Bayes recursion for the multi-object density (94) is replaced by a prediction-update recursion of the iid cluster process parameters, i.e., the cardinality $\mathrm{pmf}$ $P_{k \mid k^{\prime}}\left(n_{k}\right)$ and the PHD $D_{k \mid k^{\prime}}\left(\mathbf{x}_{k}\right)$. The mathematical details of the prediction and the update are too lengthy to repeat here, for details see [111]. Noteworthy is that the CPHD update involves a sum over all possible partitions of the set of measurements, similarly to the PHD filter update $(120)$. The complexity of this was analyzed in Section 8 , and methods to find a subset of partitions were presented in Section 9 .

\subsubsection{Implementation}

In order to implement the PHD filter, the PHD function is represented as a density mixture; a GGIW implementation was presented in [111], [154]. A comparison demonstrates that the GGIW-CPHD filter improves over the GGIW-PHD filter due to its improved cardinality estimate. Especially for settings with low probability of detection or high clutter density, the achieved performance gain is significant. However, the obtained performance gain comes with the price of an increased computational cost [111].

\subsection{POISSON MULTI-BERNOULLI MIXTURE CONJUGATE PRIOR}

The Poisson Multi-Bernoulli Mixture (PMBM) density separates the set of objects into two disjoint subsets,

$$
\mathbf{X}_{k}=\mathbf{X}_{k}^{\mathrm{d}} \uplus \mathbf{X}_{k}^{\mathrm{u}}
$$

where $\mathbf{X}_{k}^{\mathrm{d}}$ is the set of detected objects and $\mathbf{X}_{k}^{\mathrm{u}}$ is the set of undetected objects. The detected objects are objects that have been detected in at least one time step. The undetected objects are objects that have never been detected but are hypothesized to be located in the surveillance area.

Given the MEOT is based on detections, it may seem counter-intuitive to model undetected objects, however, in the PMBM filter the undetected objects are not tracked per se, it is their possible existence that is represented. Furthermore, models of undetected objects are implicitly included in other trackers as well: in a tracking algorithm, any potential object that has not been associated to a measurement is by definition 


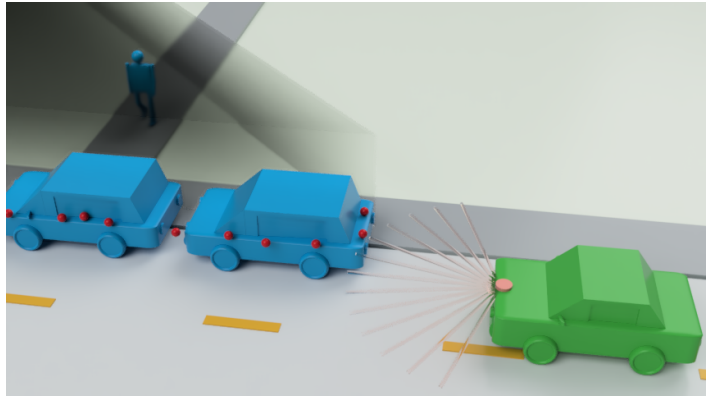

FIGURE 19. Example of the possibility of undetected objects: The cars that are parked along the street occlude the sidewalks. There might be objects, e.g., pedestrians, located in the areas behind the vehicles. In scenarios such as this one, it may be necessary for the MOT algorithm to not only track the detected objects, but also to have a representation of the areas in which undetected objects may be.

an undetected object, i.e., one that has not been detected but is hypothesized to exist.

In a tracking application the undetected model is useful, e.g., for handling tracking scenarios where there are persistent occlusions that lead to a significant probability that an object is located in the occluded region of the surveillance area, see Fig. 19. In мOT, occlusions can be modeled with the help of a non-homogeneous probability of detection $P^{\mathrm{D}}(\cdot)$ [117], [118], [155]. In case of a non-homogeneous probability of detection is employed in the PMBM filter, the undetected PPP intensity will be large in occluded areas, correctly representing that an undetected object may possibly be located there; for an example, see [103].

\subsubsection{Prediction and update}

In the PMBM density

$$
f_{k \mid k}\left(\mathbf{X}_{k}\right)=\sum_{\mathbf{X}_{k}^{\mathrm{d}} \uplus \mathbf{X}_{k}^{\mathrm{u}}=\mathbf{X}_{k}} f_{k \mid k}^{\mathrm{d}}\left(\mathbf{X}_{k}^{\mathrm{d}}\right) f_{k \mid k}^{\mathrm{u}}\left(\mathbf{X}_{k}^{\mathrm{u}}\right)
$$

the undetected objects are modeled by a PPP density $f_{k \mid k}\left(\mathbf{X}_{k}^{\mathrm{u}}\right)$, and the detected objects are modeled by an MBM density $f_{k \mid k}\left(\mathbf{X}_{k}^{\mathrm{d}}\right)$. In the MBM, each mixture component corresponds to a sequence of data associations for all measurements. The PMBM density is completely defined by the parameters

$$
\begin{aligned}
& \left\{\left(w_{k \mid k}^{\mathrm{u}, t}, p_{k \mid k}^{\mathrm{u}, t}\right)\right\}_{t \in \mathbb{T}_{k \mid k}}, \\
& \left\{\left(\mathcal{W}_{k \mid k}^{j},\left\{\left(r_{k \mid k}^{j, i}, f_{k \mid k}^{j, i}\right)\right\}_{i \in \mathbb{I}_{k \mid k}^{j}}\right)\right\}_{j \in \mathbb{J}_{k \mid k}},
\end{aligned}
$$

where (125a) are the set of undetected PPP intensity parameters, and (125b) are the set of detected MBM parameters.

Because the PMBM density is a multi-object conjugate prior [103], the prediction and Bayes update means that one computes the predicted set of parameters and posterior set of parameters, respectively. That is, the multi-object prediction and update comes down to prediction and update of singleobject densities $p_{k \mid k}^{\mathrm{u}, t}(\cdot)$ and $f_{k \mid k}^{j, i}(\cdot)$ using standard motion models (7) and measurement models (91b), for which there are many standard (nonlinear) filters, see, e.g., [8].

In the prediction, the set of parameters of the PPP birth are added to the set of parameters of the undetected PPP, this leads to an increase in the number of parameters, however, the number of MBM parameters remains constant in the PMBM prediction. The full details of the PMBM prediction are too lengthy to repeat here, see [103, Thm. 1].

In the PMBM update, the number of mixture components in the undetected PPP intensity remains constant. For a PMBM prior with an MBM

$$
f_{k \mid k-1}^{\mathrm{d}}\left(\mathbf{X}_{k}^{\mathrm{d}}\right)=\sum_{j \in \mathbb{J}_{k \mid k-1}} \mathcal{W}_{k \mid k-1}^{j} f_{k \mid k-1}^{j}\left(\mathbf{X}_{k}^{\mathrm{d}}\right)
$$

the Bayes posterior PMBM density has an MBM that is of the following format [103, Thm. 2]

$$
f_{k \mid k}^{\mathrm{d}}\left(\mathbf{X}_{k}^{\mathrm{d}}\right)=\sum_{j \in \mathbb{J}_{k \mid k-1}} \sum_{A \in \mathcal{A}^{j}} \mathcal{W}_{k \mid k}^{j, A} f_{k \mid k}^{j, A}\left(\mathbf{X}_{k}^{\mathrm{d}}\right) .
$$

In the prior 126, we have a set of predicted hypotheses, with hypothesis probabilities $w_{k \mid k-1}^{j}$ and MB densities $f_{k \mid k-1}^{j}\left(\mathbf{X}_{k}^{\mathrm{d}}\right)$. Each prior hypothesis corresponds to a sequence of data associations for all time steps up to and including time step $k-1$. The structure of the posterior MBM (127) is that for each prior hypothesis, and each possible data association, we get a posterior hypothesis, with posterior hypothesis probability $w_{k \mid k}^{j, A}$ and MB density $f_{k \mid k}^{j, A}\left(\mathbf{X}_{k}^{\mathrm{d}}\right)$. The full details of the PMBM update are too lengthy to repeat here, see [103, Thm. 2].

An interesting relationship between the PHD filter and the PMBM filter is that the PHD filter can be obtained from the PMBM filter if, after each update, the posterior PMBM is approximated as a PPP. That this is true can be understood by comparing the update of the PHD parameters $(120)$ with the updated PMBM parameters [103, Thm. 2]. With a PPP prior, the posterior is a PMB density [103, Thm. 2], i.e., a special case of the PMBM density where the MBM has a single component with posterior probability $\mathcal{W}_{k \mid k}=1$. The posterior Bernoulli parameters [103, Eq. 17] are equivalent to the updated PHD parameters 120 .

\subsubsection{Implementation}

A PMBM filter for extended objects is introduced with a GGIW implementation for the target extent in [103]. With the help of simulated data, it is shown that the extended object PMBM filter can outperform various state-of-the-art approaches, i.e., the PHD [118], CPHD [111], LMB [116] and $\delta$-GLMB filters [116]. Furthermore, based on an experiment with LIDAR data, it is illustrated that the PPP model is suitable to deal with occluded parts in the surveillance area.

In [137] the GGIW-PMBM filter was applied to mapping, and a batch measurement update was derived, using Gibbs sampling to handle the data association. Pedestrian tracking using LIDAR data, a GGIW-PMBM filter, and stochastic optimization for data association was presented in [119]. GGIWPMBM mapping using LIDAR data is presented in [156], 
where the PMBM map was later used for particle filter based localization. Joint tracking and mapping, applied to vehicle radar is presented in [157]. The PMBM filter was extended to multiple sensors and joint tracking and sensor localization in [158]. In [109] the PMBM filter was extended to the sets of trajectories framework [159]. In [160], coexisting point and extended targets are considered.

\subsection{LABELED MULTI-BERNOULLI MIXTURE CONJUGATE PRIOR}

If the standard PPP birth is replaced by an MB birth process, then a special case of the extended object PMBM filter is obtained: the MBM filter. If uniquely labeled MB birth is used (instead of PPP birth or MB birth), then a labeled MBM (LMBM) filter is obtained.

\subsubsection{Prediction and update}

An MBM density is completely defined by the parameters

$$
\left\{\left(\mathcal{W}_{k \mid k}^{j},\left\{\left(r_{k \mid k}^{j, i}, f_{k \mid k}^{j, i}\right)\right\}_{i \in \mathbb{I}_{k \mid k}^{j}}\right)\right\}_{j \in \mathbb{J}_{k \mid k}}
$$

and, analogously, an LMBM density is defined by the parameter set

$$
\left\{\left(\mathcal{W}_{k \mid k}^{j},\left\{\left(\ell^{j, i}, r_{k \mid k}^{j, i}, f_{k \mid k}^{j, i}\right)\right\}_{i \in \mathbb{I}_{k \mid k}^{j}}\right)\right\}_{j \in \mathbb{J}_{k \mid k}},
$$

where $\ell^{j, i}$ is a unique label. Note that the label $\ell^{j, i}$ is independent of the time step, in contrast to the existence probability and object state density. The first presented LMBM filter is the $\delta$-GLMB filter [115], [116].

Both the MBM density and the LMBM density are multiobject conjugate priors, and it follows that the multi-object Bayes recursion (94) comes down to prediction and update of the MBM/LMBM parameters. The prediction and update of an MBM/LMBM density is similar to the PMBM counterparts, see Section 10.3 In an LMBM filter, e.g., the $\delta$-GLMB filter [115], [116], the unique label is assigned at birth, and does not change over time, i.e., neither the prediction nor the update changes the label. Following the Bayes update, the posterior MBM/LMBM density is of the format (127). The increasing number of components in the MBM can be handled using, e.g., the data association methods presented in Section 9 .

A significant difference between the MBM filter and the PMBM filter are that in the MBM filter new Bernoullis are added in each prediction, whereas in the PMBM filter the creation of new Bernoullis is measurement driven. Measurement driven initiation of new Bernoullis (new tracks) often leads to a lower computational cost, see, e.g., [103], [161]. A further discussion of the properties of the PMBM and $\delta$-GLMB conjugate priors is provided, e.g., in [103, Sec. V.C] and [104, Sec. IV].

\subsubsection{Implementation}

The $\delta$-GLMB filter was implemented for the random matrix model with GGIW densities [116]. It turned out that the
GGIW- $\delta$-GLMB filter outperforms both the GGIW-PHD filter and the GGIW-CPHD filter, albeit with a higher computational cost. The work [103] concludes that the GGIW-PMBM filter can outperform the GGIW- $\delta$-GLMB filter both in terms of tracking performance, and in terms of computational cost.

A computationally efficient approximation of the $\delta$-GLMB filter is the so called Labeled Multi-Bernoulli (LMB) filter [116]. Given a labeled MB prior density, the posterior density is a labeled MBM density. In the LMB filter the posterior mixture is approximated by a single labeled MB density using track oriented merging, which leads to a drastic reduction in the computational cost of the tracking algorithm, without a major sacrifice in tracking performance.

The extended object $\delta$-GLMB and LMB filters have been used in several different applications: tracking cars under a rectangular extent assumption using LIDAR and RADAR [86], [162]-[164]; tracking of irregularly shaped objects using Gaussian processes or splines [165], [166]; and tracking in very high clutter levels in [167].

\subsection{JOINT PROBABILISTIC DATA ASSOCIATION}

The posterior multi-object density includes a summation over all possible data associations (95). In a Joint Probabilistic Data Association (JPDA) filter the posterior multi-object density is approximated by an ordered density

$$
p_{k \mid k^{\prime}}\left(\mathbf{X}_{k}\right) \approx \prod_{i} p_{k \mid k^{\prime}}\left(\mathbf{x}_{k}^{i}\right)
$$

where $\mathbf{x}_{k}^{i}$ is the state of the $i$ th object and the density for each object is approximated using marginal association probabilities, Section 9.4

\subsubsection{Prediction and update}

In the prediction, assuming the standard dynamic model, Section 7.2.1, each object density can be predicted independently of the other objects,

$$
p_{k+1 \mid k}\left(\mathbf{x}_{k+1}^{i}\right)=\int f_{k+1, k}\left(\mathbf{x}_{k+1}^{i} \mid \mathbf{x}_{k}^{i}\right) p_{k \mid k}\left(\mathbf{x}_{k}^{i}\right) \mathrm{d} \mathbf{x}_{k}^{i} .
$$

In the update, marginal association probabilities are first computed, see Section 9.4. Then, for each object the posterior density is approximated using the marginal association probabilities. One alternative is to approximate the posterior as

$$
p_{k \mid k}\left(\mathbf{x}_{k}^{i}\right) \approx \sum_{m \in \mathbb{M}} \beta_{i, m} p_{k \mid k}\left(\mathbf{x}_{k}^{i} \mid \mathbf{z}_{k}^{m}\right)
$$

A second alternative is that the posterior is approximated as

$$
p_{k \mid k}\left(\mathbf{x}_{k}^{i}\right) \approx \sum_{A \in \mathcal{A}} \beta_{i, \tilde{\mathbb{M}}_{A}} p_{k \mid k}\left(\mathbf{x}_{k}^{i} \mid \tilde{\mathbb{M}}_{A}\right)
$$

where, for the multi-object data association $A, \tilde{\mathbb{M}}_{A}$ is the set of measurement indices associated to object $i$. Note that $\tilde{\mathbb{M}}_{A}$ may be an empty set (object is misdetected), a singleton set 
(object detected by single measurement), or a set of cardinality $>1$ (object detected by cluster of measurements). Mixture reduction can then be applied to the posterior mixture density 132) or 133. A third alternative is that the marginal association probabilities are integrated into the update, see, e.g., [144].

\subsubsection{Implementation}

In [140] extended object JPDA is applied to data from a marine radar. A pixel-wise detector is first applied to the radar data, followed by clustering. The pre-processing with clustering allows the clusters to be fed to a standard point object JPDA filter, see, e.g., [6, Sec. 6.6, p. 350], where $M / N$ logic is used in lieu of birth and death models. The random matrix model is assumed, and GIW densities are used for the object state densities.

The joint integrated probabilistic data association (JIPDA) filter is an extension to the JPDA filter that additionally estimates existence probabilities for the objects. A multidetection JIPDA filter for extended objects was developed in [141], [168]. The developed multi-detection JIPDA filter was evaluated using an automotive radar that was mounted to a small boat, and the data was used to track two boats [141. Sec. 5]. The computational cost of the data association was alleviated by using gating and $k$-means clustering, where the number of clusters $k$ was set at least three times higher than the number of objects, i.e., over-segmentation of the data [141. Sec. 3.C]. The random matrix models was also used in this work.

In [144] marginal association probabilities (118) were used together with an orientation-axis-length parameterization of an assumed elliptic object extent. The resulting filter has a low computational cost, and compares well to the GGIW-PHD filter [114], [118].

\subsection{PROBABILISTIC MULTI HYPOTHESIS TRACKER}

The Probabilistic Multi-Hypothesis Tracker (PMHT) [169] is an approach to MOT that allows more than one measurement per object. Originally developed for point object tracking, it has also been applied to MEOT, see [170].

\subsubsection{Batch solution}

In extended object PMHT, a fixed number of objects $M$ is assumed [170, Sec. 3.A], and the following optimization problem is solved [170, Eq. 25]

$$
\hat{\mathbf{X}}_{1: T}=\arg \max _{\mathbf{X}_{1: T}} p\left(\mathbf{X}_{1: T} \mid \mathbf{Z}_{1: T}\right)
$$

where $\mathbf{Z}_{1: T}$ is the sequence of sets of measurements from time 1 to time $T$, and $\mathbf{X}_{1: T}=\left\{\mathbf{x}_{1: T}^{m}\right\}_{m=1}^{M}$ is the set of state sequences $\mathbf{x}_{1: T}^{m}$ for the $M$ objects.

The optimization problem 134 requires solving the data association problem, and in the extended object PMHT approach, Expectation Maximization (EM) is used to handle the unknown data association. The optimization problem is reformulated [170, Eq. 29]

$$
\begin{aligned}
\hat{\mathbf{X}}_{1: T} & =\arg \max _{\mathbf{X}_{1: T}} \sum_{A \in \mathcal{A}} p\left(\mathbf{X}_{1: T}, A \mid \mathbf{Z}_{1: T}\right) \\
& =\arg \max _{\mathbf{X}_{1: T}} \sum_{A \in \mathcal{A}} \log \left(p\left(\mathbf{X}_{1: T}, A, \mathbf{Z}_{1: T}\right)\right),
\end{aligned}
$$

where the data association $A$ is treated as a hidden variable in EM. Each iteration of EM consists of an expectation (E) step and a maximization (M) step. In extended object PMHT, during the E-step the posterior data association probabilities

$$
p\left(A \mid \mathbf{Z}_{1: T}, \mathbf{X}_{1: T}^{i}\right),
$$

are computed based on the current estimate $\mathbf{X}_{1: T}^{i}$. In the $\mathrm{M}$ step a new estimate is computed as

$$
\mathbf{X}_{1: T}^{(i+1)}=\arg \max _{\mathbf{X}_{1: T}} Q\left(\mathbf{X}_{1: T}, \mathbf{X}_{1: T}^{i}\right)
$$

where $Q\left(\mathbf{X}_{1: T}, \mathbf{X}_{1: T}^{i}\right)$ is an optimal lower bound [170, Eq. 30]

$$
\begin{aligned}
& Q\left(\mathbf{X}_{1: T}, \mathbf{X}_{1: T}^{i}\right) \\
& =\sum_{A \in \mathcal{A}} \log \left(p\left(\mathbf{X}_{1: T}, A, \mathbf{Z}_{1: T}\right)\right) p\left(A \mid \mathbf{Z}_{1: T}, \mathbf{X}_{1: T}^{i}\right)
\end{aligned}
$$

\subsubsection{Implementation}

In [170], the random matrix model [19] was integrated into PMHT and evaluated in simulation studies.

\subsection{GRAPH-BASED APPROACHES}

Recently, graph-based approaches to multiple object tracking [171] are becoming increasingly important. The basic idea is to formulate the multi-object tracking problem, including the unknown data association, as a factor graph. Based on the factor graph model, message passing algorithms such as the sum-product algorithm can be applied for efficient inference. For MEOT, Iterative Probabilistic Data Association (PDA) approaches are developed in [172] with the help of the sumproduct algorithm based on the assumption that the number of measurements from an object is modeled with a truncated discrete probability distribution. In [173], a MEOT method based on the sum-product algorithm is proposed for the case that the number of measurements from an object is Poisson distributed. The resulting method can detect and track multiple extended objects with a complexity that is linear in the number of measurements and quadratic in the number of objects, where no pre-processing (such as clustering) is required.

\section{CONCLUSIONS}

This tutorial gave an intuitive introduction into the topic of multiple extended object tracking (MEOT) and presented the basic state-of-the-art methods for shape estimation, data association, and track management.

MEOT is becoming increasingly important for environment perception of autonomous systems. In particular for 
autonomous driving, sensors commonly result in multiple unlabeled detections per target object, and hence, require MEOT. Compared to classical MOT for point targets, MEOT is a rather young research area that arose in the last 15 years. From a methodological point of view, MEOT is an extremely challenging problem: It involves a highly nonlinear shape estimation problem, and a huge number of possible measurement-to-target associations. Furthermore, initialization and termination of tracks can be difficult especially when only a couple of detections are available per scan. A proper target model is essential to deal with sparse and noisy data. If the measurements are not described well by the target model, data association and track management are bound to fail.

In the future, we expect that data-driven methods and machine learning techniques will become more and more import for MEOT. Instead of predefined target models, learned models that adapt to the sensors and targets will ensure more robust and precise shape estimation results. Furthermore, we expect that progress in RFS-based multi-object tracking will continue such that more precise, flexible, and efficient track management and data association techniques will be developed. Open research questions can also be found in the context of distributed and decentralized fusion of extended object estimates. Finally, with a progress in sensor and communication technology, new application areas for extended object tracking will become relevant in the future, e.g., in the context of $6 \mathrm{G}[174]$.

\section{ACKNOWLEDGEMENTS}

We would like to thank Jens Honer and Stephan Reuter for many fruitful discussions on extended object tracking and co-organizing several tutorial workshops. Furthermore, we are grateful to Hauke Kaulbersch, Simon Steuernagel, Kolja Thormann, Laura Wolf, and Shishan Yang for their valuable feedback and contributions to the source code.

\section{REFERENCES}

[1] E. Yurtsever, J. Lambert, A. Carballo, and K. Takeda, "A survey of autonomous driving: Common practices and emerging technologies," IEEE Access, vol. 8, pp. 58 443-58 469, 2020.

[2] F. Engels, P. Heidenreich, M. Wintermantel, L. Stäcker, M. Al Kadi, and A. M. Zoubir, "Automotive radar signal processing: Research directions and practical challenges," IEEE Journal of Selected Topics in Signal Processing, vol. 15, no. 4, pp. 865-878, 2021.

[3] G. Hakobyan and B. Yang, "High-performance automotive radar: A review of signal processing algorithms and modulation schemes," IEEE Signal Processing Magazine, vol. 36, no. 5, pp. 32-44, 2019.

[4] Y. Li and J. Ibanez-Guzman, "Lidar for autonomous driving: The principles, challenges, and trends for automotive lidar and perception systems," IEEE Signal Processing Magazine, vol. 37, no. 4, pp. 50-61, 2020.

[5] Y. Bar-Shalom, P. K. Willett, and X. Tian, Tracking and Data Fusion: A Handbook of Algorithms. YBS Publishing, 2011.

[6] S. Blackman and R. Popoli, Design and Analysis of Modern Tracking Systems. Norwood, MA, USA: Artech House, 1999.

[7] K. Granström, M. Baum, and S. Reuter, "Extended object tracking: Introduction, overview and applications," Journal of Advances in Information Fusion, vol. 12, no. 2, pp. 139-174, Dec. 2017.

[8] S. Särkkä, Bayesian Filtering and Smoothing. Cambridge University Press, 2013.

[9] R. Mahler, Statistical Multisource-Multitarget Information Fusion. Norwood, MA, USA: Artech House, 2007.
[10] - Advances in Multisource-Multitarget Information Fusion. Norwood, MA, USA: Artech House, 2014.

[11] A. W. M. Smeulders, D. M. Chu, R. Cucchiara, S. Calderara, A. Dehghan, and M. Shah, "Visual tracking: An experimental survey," IEEE Transactions on Pattern Analysis and Machine Intelligence, vol. 36, no. 7, pp. 1442-1468, 2014.

[12] Y. Wu, J. Lim, and M.-H. Yang, "Online object tracking: A benchmark," in IEEE Conference on Computer Vision and Pattern Recognition (CVPR), Jun. 2013.

[13] J. Janai, F. Güney, A. Behl, and A. Geiger, "Computer vision for autonomous vehicles: Problems, datasets and state of the art," Foundations and Trends ${ }^{\circledR}$ in Computer Graphics and Vision, vol. 12, no. 1-3, pp. 1308,2020 .

[14] H. Caesar, V. Bankiti, A. H. Lang, S. Vora, V. E. Liong, Q. Xu, A. Krishnan, Y. Pan, G. Baldan, and O. Beijbom, "nuScenes: A multimodal dataset for autonomous driving," in IEEE/CVF Conference on Computer Vision and Pattern Recognition (CVPR), 2020, pp. $11621-11631$.

[15] J. S. Fowdur, M. Baum, and F. Heymann, "Real-world marine radar datasets for evaluating target tracking methods," Sensors, vol. 21, no. 14, 2021.

[16] Y. Bar-Shalom, X. R. Li, and T. Kirubarajan, Estimation with Applications to Tracking and Navigation. New York, NY, USA: John Wiley \& Sons, 2001.

[17] K. Gilholm and D. Salmond, "Spatial distribution model for tracking extended objects," IEE Proceedings of Radar, Sonar and Navigation, vol. 152, no. 5, pp. 364-371, Oct. 2005.

[18] S. J. Julier and J. K. Uhlmann, "Unscented filtering and nonlinear estimation," in Proceedings of the IEEE, vol. 92, no. 3, 2004, pp. 401422 .

[19] W. Koch, "Bayesian approach to extended object and cluster tracking using random matrices," IEEE Transactions on Aerospace and Electronic Systems, vol. 44, no. 3, pp. 1042-1059, Jul. 2008.

[20] M. Feldmann, D. Fränken, and J. W. Koch, "Tracking of extended objects and group targets using random matrices," IEEE Transactions on Signal Processing, vol. 59, no. 4, pp. 1409-1420, Apr. 2011.

[21] A. K. Gupta and D. K. Nagar, Matrix variate distributions, ser. Chapman \& Hall/CRC monographs and surveys in pure and applied mathematics. Chapman \& Hall, 2000.

[22] J. Lan and X. R. Li, "Tracking of extended object or target group using random matrix: new model and approach," IEEE Transactions on Aerospace and Electronic Systems, vol. 52, no. 6, pp. 2973-2989, Dec. 2016.

[23] K. Granström and U. Orguner, "New prediction for extended targets with random matrices," IEEE Transactions on Aerospace and Electronic Systems, vol. 50, no. 2, pp. 1577-1589, Apr. 2014.

[24] N. J. Bartlett, C. Renton, and A. G. Wills, "A closed-form prediction update for extended target tracking using random matrices," IEEE Transactions on Signal Processing, vol. 68, pp. 2404-2418, 2020.

[25] N. J. Bartlett and A. G. Wills, "A robust random matrix prediction mode for extended object rotations," in 2021 24th International Conference on Information Fusion (FUSION), South Africa, Nov. 2021.

[26] G. Vivone, P. Braca, K. Granström, A. Natale, and J. Chanussot, "Converted measurements Bayesian extended target tracking applied to Xband marine radar data," Journal of Advances in Information Fusion, vol. 12, no. 2, Dec. 2017.

[27] J. Lan and X. R. Li, "Extended-object or group-target tracking using random matrix with nonlinear measurements," IEEE Transactions on Signal Processing, vol. 67, no. 19, pp. 5130-5142, Oct. 2019.

[28] Z. Li, J. Zhang, J. Wang, and Q. Zhou, "Extended object tracking using random matrix with converted measurements," IET Radar, Sonar \& Navigation, vol. 14, no. 7, pp. 981-991, Jul. 2020.

[29] _ "Recursive noise adaptive extended object tracking by variational Bayesian approximation," IEEE Access, vol. 7, pp. 151 168-151 179, 2019.

[30] Y. Xia, P. Wang, K. O. E. Berntorp, L. Svensson, K. Granström H. Mansour, P. T. Boufounos, and P. Orlik, "Learning-based extended object tracking using hierarchical truncation measurement model with automotive radar," IEEE Journal of Selected Topics in Signal Processing, 2021.

[31] L. Zhang and J. Lan, "Tracking of extended object using random matrix with non-uniformly distributed measurements," IEEE Transactions on Signal Processing, vol. 69, pp. 3812-3825, 2021. 
[32] K. Granström and J. Bramstang, "Bayesian smoothing for the extended object random matrix model," IEEE Transactions on Signal Processing, vol. 67 , no. 14, pp. 3732-3742, Jul. 2019.

[33] J. Lan and X. R. Li, "Joint tracking and classification of non-ellipsoidal extended object using random matrix," in 2014 17th International Conference on Information Fusion (FUSION), Jul. 2014.

[34] _ _ "Joint tracking and classification of extended object using random matrix," in 2013 16th International Conference on Information Fusion (FUSION), Jul. 2013, pp. 1550-1557.

[35] L. Wang, Y. Huang, R. Zhan, and J. Zhang, "Joint tracking and classification of extended targets using random matrix and Bernoulli filter for time-varying scenarios," IEEE Access, vol. 7, pp. 129 584-129603, 2019.

[36] C. Magnant, S. Kemkemian, and L. Zimmer, "Joint tracking and classification for extended targets in maritime surveillance," in 2018 IEEE Radar Conference (RadarConf18). IEEE, Apr. 2018.

[37] W. Cao, J. Lan, and X. R. Li, "Extended object tracking and classification using radar and ESM sensor data," IEEE Signal Processing Letters, vol. 25, no. 1, pp. 90-94, Jan. 2018.

[38] W. Li, Y. Jia, D. Meng, and J. Du, "Distributed tracking of extended targets using random matrices," in 2015 54th IEEE Conference on Decision and Control (CDC), Dec. 2015.

[39] G. Vivone, K. Granström, P. Braca, and P. Willett, "Multiple sensor measurement updates for the extended target tracking random matrix model," IEEE Transactions on Aerospace and Electronic Systems, vol. 53, no. 5, pp. 2544-2558, Oct. 2017.

[40] J. Liu and G. Guo, "Distributed asynchronous extended target tracking using random matrix," IEEE Sensors Journal, vol. 20, no. 2, pp. 947956, Jan. 2020.

[41] M. Baum, F. Faion, and U. D. Hanebeck, "Modeling the target extent with multiplicative noise," in 2012 15th International Conference on Information Fusion, 2012, pp. 2406-2412.

[42] S. Yang and M. Baum, "Tracking the orientation and axes lengths of an elliptical extended object," IEEE Transactions on Signal Processing, vol. 67 , no. 18 , pp. $4720-4729$, Sep. 2019.

[43] F. Govaers, "On independent axes estimation for extended target tracking," in 2019 Sensor Data Fusion: Trends, Solutions, Applications (SDF), 2019, pp. 1-6.

[44] J. S. Fowdur, M. Baum, and F. Heymann, "Tracking targets with known spatial extent using experimental marine radar data," in 201922 th International Conference on Information Fusion (FUSION), 2019, pp. 1-8.

[45] M. Li, J. Lan, and X. R. Li, "Tracking of elliptical extended object with unknown but fixed lengths of axes," in 2020 IEEE 23rd International Conference on Information Fusion (FUSION), Virtual, Jul. 2020.

[46] B. Tuncer and E. Özkan, "Random matrix based extended target tracking with orientation: A new model and inference," IEEE Transactions On Signal Processing, vol. 69, pp. 1910-1923, 2021.

[47] S. Liu, Y. Liang, L. Xu, T. Li, and X. Hao, "EM-based extended object tracking without a priori extension evolution model," Signal Processing, vol. 188, Nov. 2021.

[48] M. Baum and U. D. Hanebeck, "Extended object tracking with random hypersurface models," IEEE Transactions on Aerospace and Electronic Systems, vol. 50, no. 1, pp. 149-159, 2014.

[49] K. Thormann, S. Yang, and M. Baum, "A comparison of Kalman filterbased approaches for elliptic extended object tracking," in 2020 IEEE 23rd International Conference on Information Fusion (FUSION), Virtual, Jul. 2020.

[50] G. Yao, R. Saltus, and A. Dani, "Image moment-based extended object tracking for complex motions," IEEE Sensors Journal, vol. 20, no. 12, pp. 6560-6572, Jun. 2020.

[51] S. Labsir, A. Giremus, B. Yver, and T. Benoudiba-Campanini, "Joint shape and centroid position tracking of a cluster of space debris by filtering on lie groups," Signal Processing, vol. 183, p. 108027, 2021.

[52] L. A. Giefer, J. Clemens, and K. Schill, "Extended object tracking on the affine group Aff (2)," in 2020 IEEE 23rd International Conference on Information Fusion (FUSION), Virtual, Jul. 2020, pp. 1-8.

[53] K. Granström, C. Lundquist, and U. Orguner, "Tracking rectangular and elliptical extended targets using laser measurements," in 14th International Conference on Information Fusion, Chicago, IL, USA, Jul. 2011, pp. 592-599.

[54] P. Brosseit, B. Duraisamy, and J. Dickmann, "The volcanormal density for radar-based extended target tracking," in 2017 IEEE 20th International Conference on Intelligent Transportation Systems (ITSC), Oct. 2017.
[55] Y. Ren and W. Xia, "Distributed extended object tracking based on diffusion strategy," in 2020 28th European Signal Processing Conference (EUSIPCO), 2021, pp. 2338-2342.

[56] K. Thormann and M. Baum, "Fusion of elliptical extended object estimates parameterized with orientation and axes lengths," IEEE Transactions on Aerospace and Electronic Systems, vol. 57, no. 4, pp. 2369-2382, 2021.

[57] F. Faion, A. Zea, M. Baum, and U. D. Hanebeck, "Partial likelihood for unbiased extended object tracking," in 2015 18th International Conference on Information Fusion (FUSION), 2015, pp. 1022-1029.

[58] H. Kaulbersch, M. Baum, and P. Willett, "An EM approach for contour tracking based on point clouds," in 2016 IEEE International Conference on Multisensor Fusion and Integration for Intelligent Systems (MFI), Baden-Baden, Germany, Sep. 2016, pp. 529-533.

[59] H. Kaulbersch, M. Baum, and P. Willett, "EM approach for tracking star-convex extended objects," in 2017 20th International Conference on Information Fusion (FUSION), Jul. 2017.

[60] X. Tang, M. Li, R. Tharmarasa, and T. Kirubarajan, "Seamless tracking of apparent point and extended targets using Gaussian process PMHT," IEEE Transactions on Signal Processing, vol. 67, no. 18, pp. 4825-4838, Sep. 2019.

[61] N. Wahlström and E. Özkan, "Extended target tracking using Gaussian processes," IEEE Transactions on Signal Processing, vol. 63, no. 16, pp. 4165-4178, Aug. 2015.

[62] Z. Zhang, "Parameter Estimation Techniques: A Tutorial with Application to Conic Fitting," Image and Vision Computing, vol. 15, no. 1, pp. 59-76, 1997.

[63] N. Chernov, Circular and Linear Regression: Fitting Circles and Lines by Least Squares. CRC Press, 2010.

[64] M. Baum, Simultaneous tracking and shape estimation of extended objects. KIT Scientific Publishing, 2013, vol. 13.

[65] A. Zea, F. Faion, M. Baum, and U. D. Hanebeck, "Level-set random hypersurface models for tracking nonconvex extended objects," IEEE Transactions on Aerospace and Electronic Systems, vol. 52, no. 6, pp. 2990-3007, 2016.

[66] C. E. Rasmussen and C. K. I. Williams, Gaussian processes for machine learning. Boston, MA, USA: MIT Press, 2006.

[67] H. Kaulbersch, J. Honer, and M. Baum, "A Cartesian B-spline vehicle model for extended object tracking," in 201821 st International Conference on Information Fusion (FUSION), 2018.

[68] M. Kumru and E. Özkan, "Three-dimensional extended object tracking and shape learning using Gaussian processes," IEEE Transactions on Aerospace and Electronic Systems, vol. 57, no. 5, pp. 2795-2814, 2021.

[69] F. Ebert and H. Wuensche, "Dynamic object tracking and 3d surface estimation using Gaussian processes and extended Kalman filter," in 2019 IEEE Intelligent Transportation Systems Conference (ITSC), Oct. 2019.

[70] B. Naujoks, P. Burger, and H. Wuensche, "Fast 3D extended target tracking using NURBS surfaces," in 2019 IEEE Intelligent Transportation Systems Conference (ITSC), Oct. 2019, pp. 1104-1109.

[71] T. Baur, J. Reuter, A. Zea, and U. D. Hanebeck, "Shape estimation and tracking using spherical double Fourier series for three-dimensional range sensors," in Proceedings of the 2021 IEEE International Conference on Multisensor Fusion and Integration for Intelligent Systems (MFI 2021), Karlsruhe, September 2021.

[72] M. Michaelis, P. Berthold, T. Luettel, D. Meissner, and H. J. Wuensche, "Extended object tracking with an improved measurement-to-contour association," in 2020 IEEE 23rd International Conference on Information Fusion (FUSION), Virtual, Jul. 2020, pp. 1-6.

[73] M. Kumru, H. Köksal, and E. Özkan, "Variational measurement update for extended object tracking using Gaussian processes," IEEE Signal Processing Letters, vol. 28, pp. 538-542, 2021.

[74] H. Alqaderi, F. Govaers, and W. Koch, "Symmetric star-convex shape tracking with Wishart filter," in 2021 24th International Conference on Information Fusion (FUSION), South Africa, Nov. 2021.

[75] X. Cao, J. Lan, and X. R. Li, "Extension-deformation approach to extended object tracking," IEEE Transactions on Aerospace and Electronic Systems, vol. 57, no. 2, pp. 866-881, 2021

[76] K. Thormann, M. Baum, and J. Honer, "Extended target tracking using Gaussian processes with high-resolution automotive radar," in $201821 \mathrm{st}$ International Conference on Information Fusion (FUSION), Jul. 2018, pp. 1764-1770.

[77] H. Yu, W. An, and R. Zhu, "Extended target tracking and feature estimation for optical sensors based on the Gaussian process," Sensors, vol. 19, no. 7, p. 1704, Apr. 2019. 
[78] S. Särkkä, A. Solin, and J. Hartikainen, "Spatiotemporal learning via infinite-dimensional Bayesian filtering and smoothing: A look at Gaussian process regression through Kalman filtering," IEEE Signal Processing Magazine, vol. 30, no. 4, pp. 51-61, Jul. 2013.

[79] W. Aftab, R. Hostettler, A. De Freitas, M. Arvaneh, and L. Mihaylova, "Spatio-temporal Gaussian process models for extended and group object tracking with irregular shapes," IEEE Transactions on Vehicular Technology, vol. 68, no. 3, pp. 2137-2151, 2019.

[80] B. Tuncer, M. Kumru, and E. Özkan, "Extended target tracking and classification using neural networks," in 2019 22th International Conference on Information Fusion (FUSION), 2019.

[81] S. Lee and J. McBride, "Extended object tracking via positive and negative information fusion," IEEE Transactions on Signal Processing, vol. 67, no. 7, pp. 1812-1823, Apr. 2019.

[82] A. Zea, F. Faion, J. Steinbring, and U. D. Hanebeck, "Exploiting negative measurements for tracking star-convex extended objects," in 2016 IEEE International Conference on Multisensor Fusion and Integration for Intelligent Systems (MFI), Baden-Baden, Germany, Sep. 2016, pp. 622628.

[83] A. Zea, F. Faion, and U. D. Hanebeck, "Exploiting clutter: Negative information for enhanced extended object tracking," in Proceedings of the 18th International Conference on Information Fusion (Fusion 2015), Washington D. C., USA, Jul. 2015.

[84] M. Michaelis, P. Berthold, T. Luettel, D. Meissner, and H. Wuensche, "A merging strategy for Gaussian process extended target estimates in multisensor applications," in 2019 IEEE Intelligent Vehicles Symposium (IV), Jun. 2019, pp. 1803-1808.

[85] H. Kaulbersch, J. Honer, and M. Baum, "EM-based extended target tracking with automotive radar using learned spatial distribution models," in 2019 22th International Conference on Information Fusion (FUSION), 2019, pp. 1-8.

[86] A. Scheel and K. Dietmayer, "Tracking multiple vehicles using a variational radar model," IEEE Transactions on Intelligent Transportation Systems, vol. 20, no. 10, pp. 3721-3736, Oct. 2019.

[87] C. M. Bishop, Pattern recognition and machine learning. New York, USA: Springer, 2006.

[88] J. Honer and H. Kaulbersch, "Bayesian extended target tracking with automotive radar using learned spatial distribution models," in 2020 IEEE International Conference on Multisensor Fusion and Integration for Intelligent Systems (MFI), 2020, pp. 316-322.

[89] G. Yao, P. Wang, K. Berntorp, H. Mansour, P. Boufounos, and P. V. Orlik, "Extended object tracking with automotive radar using B-spline chained ellipses model," in ICASSP 2021 - 2021 IEEE International Conference on Acoustics, Speech and Signal Processing (ICASSP), 2021, pp. 84088412.

[90] — , "Extended object tracking with spatial model adaptation using automotive radar," in 2021 24th International Conference on Information Fusion (FUSION), South Africa, Nov. 2021.

[91] X. Cao, J. Lan, X. R. Li, and Y. Liu, "Automotive radar-based vehicle tracking using data-region association," IEEE Transactions on Intelligent Transportation Systems, pp. 1-14, 2021.

[92] G. Peyré and M. Cuturi, "Computational optimal transport: With applications to data science," Foundations and Trends ${ }^{\circledR}$ in Machine Learning, vol. 11, no. 5-6, pp. 355-607, 2019.

[93] D. Huttenlocher, G. Klanderman, and W. Rucklidge, "Comparing images using the Hausdorff distance," IEEE Transactions on Pattern Analysis and Machine Intelligence, vol. 15, no. 9, pp. 850-863, 1993.

[94] S. Yang, M. Baum, and K. Granström, "Metrics for performance evaluation of elliptic extended object tracking methods," in 2016 IEEE International Conference on Multisensor Fusion and Integration for Intelligent Systems (MFI), Baden-Baden, Germany, Sep. 2016, pp. 523-528.

[95] L. Sun, S. Zhang, B. Ji, and J. Pu, "Performance evaluation for shape estimation of extended objects using a modified Hausdorff distance," in 2016 IEEE International Conference on Information and Automation (ICIA), 2016, pp. 780-784.

[96] D. Schuhmacher, B.-T. Vo, and B.-N. Vo, "A consistent metric for performance evaluation of multi-object filters," IEEE Transactions on Signal Processing, vol. 56, no. 8, pp. 3447-3457, Aug. 2008.

[97] H. W. Kuhn, "The Hungarian method for the assignment problem," Naval Research Logistics Quarterly, no. 2, pp. 79-86, 1955.

[98] J. Munkres, "Algorithms for the assignment and transportation problems," Journal of the Society for Industrial and Applied Mathematics, vol. 5, no. 1, pp. 32-38, Mar. 1957.
[99] R. Jonker and A. Volgenant, "A shortest augmenting path algorithm for dense and sparse linear assignment problems," Computing, vol. 38, no. 4, pp. 325-340, Dec. 1987.

[100] C. Wolf and J.-M. Jolion, "Object count/area graphs for the evaluation of object detection and segmentation algorithms," Int. J. Doc. Anal. Recognit., vol. 8, no. 4, p. 280-296, Sep. 2006.

[101] M. Levandowsky and D. Winter, "Distance between sets," Nature, vol. 234, no. 5323, pp. 34-35, Nov. 1971.

[102] M. Fröhle, , K. Granström, and H. Wymeersch, "Decentralized Poisson multi-Bernoulli filtering for vehicle tracking," IEEE Access, vol. 8, 2020.

[103] K. Granström, M. Fatemi, and L. Svensson, "Poisson multi-Bernoull conjugate prior for multiple extended object estimation," IEEE Transactions on Aerospace and Electronic Systems, vol. 56, no. 1, pp. 208-225, Feb. 2020.

[104] A. F. García-Fernández, J. Williams, K. Granström, and L. Svensson, "Poisson multi-Bernoulli mixture filter: direct derivation and implementation," IEEE Transactions on Aerospace and Electronic Systems, vol. 54, no. 4, Aug. 2018.

[105] J. Williams, "Marginal multi-Bernoulli filters: RFS derivation of MHT, JIPDA, and association-based member," IEEE Transactions on Aerospace and Electronic Systems, vol. 51, no. 3, pp. 1664-1687, Jul. 2015

[106] Y. Xia, K. Granström, L. Svensson, and A. F. G. Fernández, "Performance evaluation of multi-Bernoulli conjugate priors for multi-target filtering," in 2017 20th International Conference on Information Fusion (FUSION), Xi'an, China, Jul. 2017.

[107] Y. Xia, K. Granström, L. Svensson, M. Fatemi, A. F. García-Fernández, and J. L. Williams, "Poisson multi-Bernoulli approximations for multiple extended object filtering," IEEE Transactions on Aerospace and Electronic Systems, pp. 1-1, 2021.

[108] Y. Xia, K. Granström, L. Svensson, and A. F. G. Fernández, "An implementation of the Poisson multi-Bernoulli mixture trajectory filter via dual decomposition," in 2018 21st International Conference on Information Fusion (FUSION), Cambridge, UK, Jul. 2018.

[109] Y. Xia, K. Granström, L. Svensson, A. F. García-Fernández, and J. L. Williams, "Extended target Poisson multi-Bernoulli mixture trackers based on sets of trajectories," in 2019 22th International Conference on Information Fusion (FUSION), Ottawa, Canada, Jul. 2019.

[110] —, "Multi-scan implementation of the trajectory Poisson multiBernoulli mixture filter," Journal of Advances in Information Fusion, vol. 14, no. 2, pp. 213-235, 2019.

[111] C. Lundquist, K. Granström, and U. Orguner, "An extended target CPHD filter and a Gamma Gaussian inverse Wishart implementation," IEEE Journal of Selected Topics in Signal Processing, Special Issue on Multitarget Tracking, vol. 7, no. 3, pp. 472-483, Jun. 2013.

[112] K. Granström and U. Orguner, "On spawning and combination of extended/group targets modeled with random matrices," IEEE Transactions on Signal Processing, vol. 61, no. 3, pp. 678-692, Feb. 2013.

[113] K. Gilholm, S. Godsill, S. Maskell, and D. Salmond, "Poisson models for extended target and group tracking," in Proceedings of Signal and Data Processing of Small Targets, vol. 5913. San Diego, CA, USA: SPIE, Aug. 2005, pp. 230-241.

[114] K. Granström and U. Orguner, "Estimation and maintenance of measurement rates for multiple extended target tracking," in 2012 15th International Conference on Information Fusion, Singapore, Jul. 2012, pp. 2170-2176.

[115] M. Beard, S. Reuter, K. Granström, B.-T. Vo, B.-N. Vo, and A. Scheel, "A generalised labelled multi-Bernoulli filter for extended multi-target tracking," in 2015 18th International Conference on Information Fusion (FUSION), Washington, DC, USA, Jul. 2015, pp. 991-998.

[116] —, "Multiple extended target tracking with labelled random finite sets," IEEE Transactions on Signal Processing, vol. 64, no. 7, pp. 1638 1653, Apr. 2016.

[117] K. Granström, C. Lundquist, and U. Orguner, "Extended target tracking using a Gaussian mixture PHD filter," IEEE Transactions on Aerospace and Electronic Systems, vol. 48, no. 4, pp. 3268-3286, Oct. 2012.

[118] K. Granström and U. Orguner, "A PHD filter for tracking multiple extended targets using random matrices," IEEE Transactions on Signal Processing, vol. 60, no. 11, pp. 5657-5671, Nov. 2012.

[119] K. Granström, L. Svensson, S. Reuter, Y. Xia, and M. Fatemi, "Likelihood-based data association for extended object tracking using sampling methods," IEEE Transactions on Intelligent Vehicles, vol. 3, no. 1, Mar. 2018. 
[120] R. L. Graham, D. E. Knuth, and O. Patashnik, Concrete Mathematics Reading, MA, USA: Addison-Wesley, 1988.

[121] G.-C. Rota, "The number of partitions of a set," The American Mathematical Monthly, vol. 71, no. 5, pp. 498-504, May 1964.

[122] Y. Bar-Shalom and W. D. Blair, Multitarget-multisensor tracking: applications and advances, vol III. Artech House, 2000.

[123] K. Granström and C. Lundquist, "On the use of multiple measurement models for extended target tracking," in 2013 16th International Conference on Information Fusion, Istanbul, Turkey, Jul. 2013, pp. 1534-1541.

[124] K. Granström, S. Reuter, D. Meissner, and A. Scheel, "A multiple model PHD approach to tracking of cars under an assumed rectangular shape," in Proceedings of the International Conference on Information Fusion, Salamanca, Spain, Jul. 2014.

[125] K. Granström, A. Natale, P. Braca, G. Ludeno, and F. Serafino, "Gamma Gaussian inverse Wishart probability hypothesis density for extended target tracking using X-band marine radar data," IEEE Transactions on Geoscience and Remote Sensing, vol. 53, no. 12, pp. 6617-6631, Dec. 2015.

[126] K. Granström, U. Orguner, R. Mahler, and C. Lundquist, "Corrections on: "extended target tracking using a Gaussian-mixture PHD filter"," IEEE Transactions on Aerospace and Electronic Systems, vol. 53, no. 2, pp. 1055-1058, Dec. 2017.

[127] M. Ester, H.-P. Kriegel, J. Sander, and X. Xu, "A density-based algorithm for discovering clusters in large spatial databases with noise," in Proceedings of AAAI. AAAI Press, 1996, pp. 226-231.

[128] J. Yang, F. Liu, H. Ge, and Y. Yuan, "Multiple extended target tracking algorithm based on GM-PHD filter and spectral clustering," EURASIP Journal on Advances in Signal Processing, Jul. 2014.

[129] Y. Zhang and H. Ji, "A novel fast partitioning algorithm for extended target tracking using a Gaussian mixture PHD filter," Signal Processing, vol. 93, no. 11, pp. 2975-2985, 2013.

[130] — , "Robust Bayesian partition for extended target Gaussian inverse Wishart PHD filter," IET Signal Processing, vol. 8, no. 4, pp. 330-338, Jun. 2014.

[131] D. Bertsekas, "The auction algorithm: A distributed relaxation method for the assignment problem," Annals of Operations Research, vol. 14, no. 1 , pp. $105-123,1988$.

[132] K. Murty, "An algorithm for ranking all the assignments in order of increasing cost," Operations Research, vol. 16, no. 3, pp. 682-687, 1968.

[133] D. F. Crouse, "On implementing 2d rectangular assignment algorithms," IEEE Transactions on Aerospace and Electronic Systems, vol. 52, no. 4, pp. 1679-1696, Aug. 2016.

[134] S. Geman and D. Geman, "Stochastic relaxation, Gibbs distributions, and the Bayesian restoration of images," IEEE Transactions on Pattern Analysis and Machine Intelligence, vol. 6, no. 6, pp. 721-741, 1984.

[135] B. N. Vo, B. T. Vo, and H. G. Hoang, "An efficient implementation of the generalized labeled multi-Bernoulli filter," IEEE Transactions on Signal Processing, vol. 65, no. 8, pp. 1975-1987, Apr. 2017.

[136] S. Reuter, A. Danzer, M. Stübler, A. Scheel, and K. Granström, "A fast implementation of the labeled multi-Bernoulli filter using Gibbs sampling," in 2017 IEEE Intelligent Vehicles Symposium (IV), Redondo Beach, CA, USA, Jun. 2017, pp. 765-772.

[137] M. Fatemi, K. Granström, L. Svensson, F. Ruiz, and L. Hammarstrand, "Poisson multi-Bernoulli mapping using Gibbs sampling," IEEE Transactions on Signal Processing, vol. 65, no. 11, pp. 2814-2827, Jun. 2017.

[138] J. Honer and F. Schmieder, "Gibbs sampling of measurement partitions and associations for extended multi-target tracking," in 2019 22th International Conference on Information Fusion (FUSION), Ottawa, Canada, Jul. 2019, pp. 1-8.

[139] Y. Bar-Shalom, F. Daum, and J. Huang, "The probabilistic data association filter," IEEE Control Systems, vol. 29, no. 6, pp. 82-100, Dec. 2009.

[140] G. Vivone and P. Braca, "Joint probabilistic data association tracker for extended target tracking applied to X-band marine radar data," IEEE Journal of Oceanic Engineering, vol. 41, no. 4, pp. 1007-1019, Oct. 2016.

[141] M. Schuster, J. Reuter, and G. Wanielik, "Multi detection joint integrated probabilistic data association using random matrices with applications to radar-based multi-object tracking," Journal of Advances in Information Fusion, vol. 12, no. 2, pp. 175-188, Dec. 2017.

[142] K. Romeo, D. F. Crouse, Y. Bar-Shalom, and P. Willett, "The JPDAF in practical systems: approximations," in Proceedings of Signal and Data Processing of Small Targets, Orlando, FL, USA, 2010.
[143] F. Meyer, Z. Liu, and M. Z. Win, "Scalable probabilistic data association with extended objects," in IEEE International Conference on Communications Workshops, May 2019, pp. 1-6.

[144] S. Yang, K. Thormann, and M. Baum, "Linear-time joint probabilistic data association for multiple extended object tracking," in 2018 IEEE 10th Sensor Array and Multichannel Signal Processing Workshop (SAM), 2018.

[145] S. Yang, L. M. Wolf, and M. Baum, "Marginal association probabilities for multiple extended objects without enumeration of measurement partitions," in 23rd International Conference on Information Fusion (Fusion 2020), Virtual, Jul. 2020.

[146] R. Mahler, "PHD filters for nonstandard targets, I: Extended targets," in 2009 12th International Conference on Information Fusion, Seattle, WA, USA, Jul. 2009, pp. 915-921.

[147] A. Swain and D. Clark, "Extended object filtering using spatial independent cluster processes," in 2010 13th International Conference on Information Fusion, Edinburgh, UK, Jul. 2010, pp. 1-8.

[148] — - "The PHD filter for extended target tracking with estimable extent shape parameters of varying size," in 2012 15th International Conference on Information Fusion, Singapore, Jul. 2012, pp. 1111-1118.

[149] K. Granström and U. Orguner, "Implementation of the GIW-PHD filter," Department of Electrical Engineering, Linköping University, SE-581 83 Linköping, Sweden, Tech. Rep. LiTH-ISY-R-3046, Mar. 2012. [Online]. Available: http://urn.kb.se/resolve?urn=urn:nbn:se:liu:diva-94585

[150] H. Zhang, H. Xu, X.-Y. Wang, and W. An, "A PHD filter for tracking closely spaced objects with elliptic random hypersurface models," in Proceedings of the 16th International Conference on Information Fusion, 2013, pp. 1558-1565.

[151] L. Sun, H. Yu, J. Lan, Z. Fu, Z. He, and J. Pu, "Tracking of multiple maneuvering random hypersurface extended objects using high resolution sensors," Remote Sensing, vol. 13, no. 15, 2021.

[152] T. Baur, J. Böhler, S. Wirtensohn, and J. Reuter, "Tracking of spline modeled extended targets using a Gaussian mixture PHD filter," in 2019 22th International Conference on Information Fusion (FUSION), 2019, pp. $1-8$.

[153] J. Sjudin, M. Marcusson, L. Svensson, and L. Hammarstrand, "Extended object tracking using sets of trajectories with a PHD filter," in 2021 24th International Conference on Information Fusion (FUSION), South Africa, Nov. 2021

[154] U. Orguner, C. Lundquist, and K. Granström, "Extended target tracking with a cardinalized probability hypothesis density filter," in 14th International Conference on Information Fusion, Chicago, IL, USA, Jul. 2011, pp. $65-72$

[155] K. Wyffels and M. Campbell, "Negative information for occlusion reasoning in dynamic extended multiobject tracking," IEEE Transactions on Robotics, vol. 31, no. 2, pp. 425-442, Apr. 2015.

[156] P. Kielen and M. Olsson, "Mapping and localization using automotive lidar - Poisson multi-Bernoulli mapping and marginalized particle filter localization,” Master's thesis, Chalmers tekniska högskola, 2017. [Online]. Available: https://hdl.handle.net/20.500.12380/252489

[157] G. Iturbe Otegui, "Tracking stationary and moving extended objects in dense urban scenarios - an application of the PMBM filter with stochastic optimization to radar data," Master's thesis, Chalmers tekniska högskola, 2018. [Online]. Available: https://hdl.handle.net/20. $500.12380 / 256107$

[158] M. Fröhle, C. Lindberg, K. Granström, and H. Wymeersch, "Multisensor Poisson multi-Bernoulli filter for joint target-sensor state tracking," IEEE Transactions on Intelligent Vehicles, vol. 4, no. 4, pp. 609-621, 2019.

[159] A. F. García-Fernández, L. Svensson, and M. R. Morelande, "Multiple target tracking based on sets of trajectories," IEEE Transactions on Aerospace and Electronic Systems, vol. 56, no. 3, pp. 1685-1707, 2020.

[160] A. F. García-Fernández, J. L. Williams, L. Svensson, and Y. Xia, "A Poisson multi-Bernoulli mixture filter for coexisting point and extended targets," IEEE Transactions on Signal Processing, vol. 69, pp. 26002610, 2021.

[161] L. Cament, M. Adams, and J. Correa, "A multi-sensor, Gibbs sampled, implementation of the multi-Bernoulli Poisson filter," in 2018 21 st International Conference on Information Fusion (FUSION), Cambridge, UK, Jul. 2018, pp. 2580-2587.

[162] A. Scheel, S. Reuter, and K. Dietmayer, "Using separable likelihoods for laser-based vehicle tracking with a labeled multi-Bernoulli filter," in 2016 19th International Conference on Information Fusion (FUSION), Heidelberg, Germany, Jul. 2016, pp. 1200-1207. 
[163] A. Scheel, C. Knill, S. Reuter, and K. Dietmayer, "Multi-sensor multiobject tracking of vehicles using high-resolution radars," in 2016 IEEE Intelligent Vehicles Symposium (IV), Jun. 2016, pp. 558-565.

[164] A. Scheel, S. Reuter, and K. Dietmayer, "Vehicle tracking using extended object methods: An approach for fusing radar and laser," in 2017 IEEE International Conference on Robotics and Automation (ICRA), Singapore, Jun. 2017, pp. 231-238.

[165] T. Hirscher, A. Scheel, S. Reuter, and K. Dietmayer, "Multiple extended object tracking using Gaussian processes," in 2016 19th International Conference on Information Fusion (FUSION), Jul. 2016, pp. 868-875.

[166] A. Daniyan, S. Lambotharan, A. Deligiannis, Y. Gong, and W.-H. Chen, "Bayesian multiple extended target tracking using labeled random finite sets and splines," IEEE Transactions on Signal Processing, vol. 66, no. 22, pp. 6076-6091, Nov. 2018.

[167] S. Reuter, M. Beard, K. Granstrom, and K. Dietmayer, "Tracking extended targets in high clutter using a GGIW-LMB filter," in 2015 Sensor Data Fusion: Trends, Solutions, Applications (SDF), Oct. 2015, pp. 1-6.

[168] M. Schuster, J. Reuter, and G. Wanielik, "Probabilistic data association for tracking extended targets under clutter using random matrices," in 2015 18th International Conference on Information Fusion (FUSION), Washington, DC, USA, Jul. 2015, pp. 961-968.

[169] R. Streit and T. E. Luginbuhl, "A probabilistic multihypothesis tracking algorithm without enumeration and pruning," in In proceedings of the Sixth Joint Service Data Fusion Symposium, Laurel, MD, USA, Jun. 1993, pp. 1015-1024.

[170] M. Wieneke and W. Koch, "A PMHT approach for extended objects and object groups," IEEE Transactions on Aerospace and Electronic Systems, vol. 48 , no. 3, pp. 2349-2370, 2012

[171] F. Meyer, T. Kropfreiter, J. L. Williams, R. Lau, F. Hlawatsch, P. Braca, and M. Z. Win, "Message passing algorithms for scalable multitarge tracking," Proceedings of the IEEE, vol. 106, no. 2, pp. 221-259, 2018.

[172] F. Meyer and M. Z. Win, "Scalable data association for extended object tracking," IEEE Transactions on Signal and Information Processing over Networks, vol. 6, pp. 491-507, 2020.

[173] F. Meyer and J. L. Williams, "Scalable detection and tracking of geometric extended objects," IEEE Transactions on Signal Processing, vol. 69 pp. 6283-6298, 2021.

[174] C. De Lima, D. Belot, R. Berkvens, A. Bourdoux, D. Dardari, M. Guillaud, M. Isomursu, E.-S. Lohan, Y. Miao, A. N. Barreto, M. R. K. Aziz, J. Saloranta, T. Sanguanpuak, H. Sarieddeen, G. Seco-Granados, J. Suutala, T. Svensson, M. Valkama, B. Van Liempd, and H. Wymeersch, "Convergent communication, sensing and localization in 6G systems: An overview of technologies, opportunities and challenges," IEEE Access, vol. 9 , pp. 26902-26925, 2021

\section{BIOGRAPHIES}

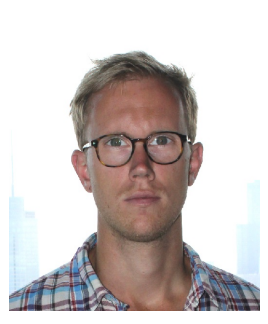

KARL GRANSTRÖM received the M.Sc. degree in applied physics and electrical engineering and the Ph.D. degree in automatic control from Linköping University, Sweden, in May 2008 and November 2012, respectively. He is currently Tech Lead for Object Tracking at Embark Trucks Inc., San Francisco, CA, USA. Before joining Embark, he worked at the Department of Electrical Engineering, Chalmers University of Technology, Gothenburg, Sweden, first as a Postdoctoral Researcher and then as a Researcher. He held postdoctoral positions at the Department of Electrical and Computer Engineering, University of Connecticut, USA, from September 2014 to August 2015, and at the Department of Electrical Engineering, Linköping University, from December 2012 to August 2014. His research interests include estimation theory, multiple model estimation, sensor fusion, and target tracking, especially for extended targets. He received paper awards at the Fusion 2011 and Fusion 2012 conferences. He has organized several workshops and tutorials on the topic of Multiple Extended Target Tracking and Sensor Fusion. At Fusion 2018, the International Society for Information Fusion (ISIF) awarded him the ISIF Young Investigator Award for his contributions to extended target tracking research and his services to the research community.

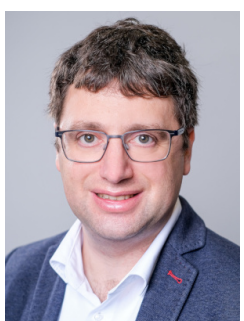

MARCUS BAUM received his Diploma degree in 2007 and his Ph.D. degree in 2013, both in Computer Science from the Karlsruhe Institute of Technology (KIT), Germany. He is Professor of Computer Science and Head of the Data Fusion Lab at the University of Goettingen, Germany. His research interests are in the areas of signal processing, state estimation, machine learning, sensor data fusion, tracking, and environment perception. $\mathrm{He}$ is Area Editor of the Journal of Advances in Information Fusion (JAIF) and Associate Editor of Letters of the IEEE Transactions on Aerospace and Electronic Systems (TAES). He was Local Arrangements Co-Chair of the 2016 International Conference on Information Fusion (FUSION) and Technical Co-Chair of the 2016, 2020, and 2021 IEEE International Conference on Multisensor Fusion and Integration for Intelligent Systems (MFI). He has organized several special sessions, workshops, and tutorials in the area of (multiple) extended object tracking and sensor fusion. He received the best student paper award at the FUSION 2011 conference, and the International Society for Information Fusion (ISIF) awarded him the 2017 ISIF Young Investigator Award. 